\title{
IntechOpen
}

\section{Psycho-Social Aspects of Human Sexuality and Ethics}

\author{
Edited by Dhastagir Sultan Sheriff
}





\section{Psycho-Social Aspects of Human Sexuality and Ethics}

Edited by Dhastagir Sultan Sheriff 

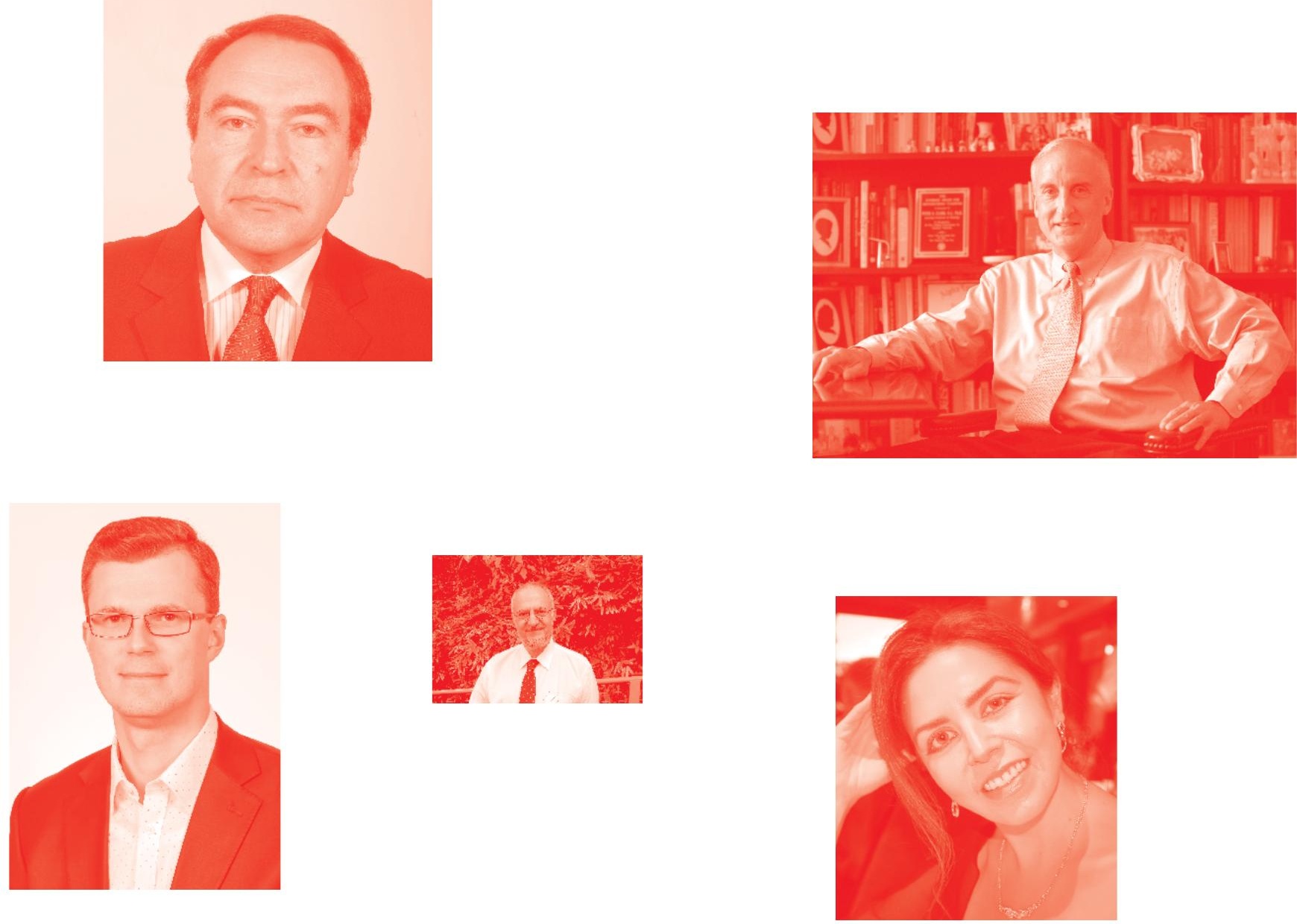

Supporting open minds since 2005
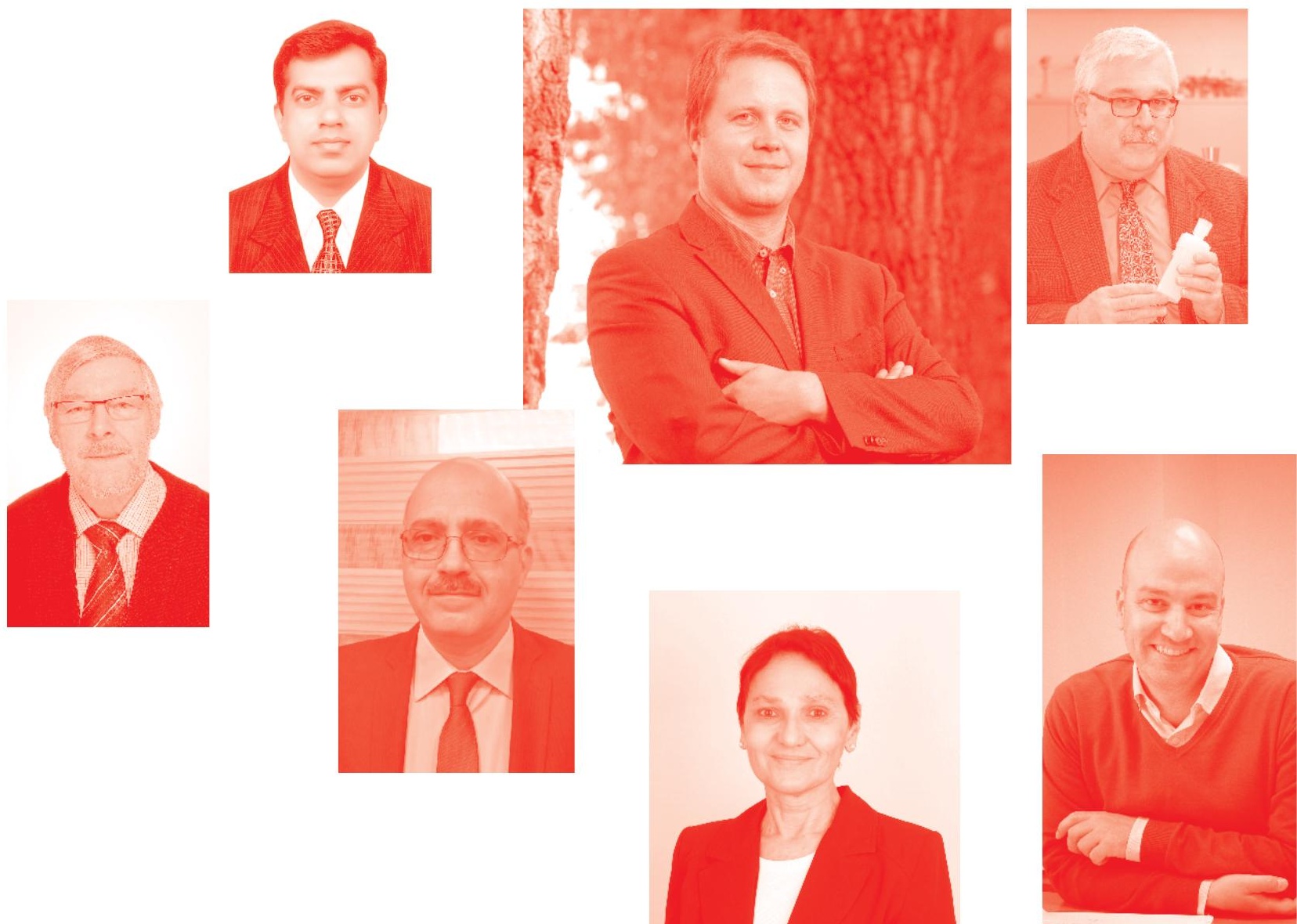
Psycho-Social Aspects of Human Sexuality and Ethics

http: //dx. doi.org/10.5772/intechopen. 73465

Edited by Dhastagir Sultan Sheriff

Scientific Contributor: Mihaela Laura Vica

\section{Contributors}

Kari Schneider, Rachel Cafferty, Joanna Ekstrom, William Elwood, Craig Nagoshi, Catherine Baggett, Julie Nagoshi, Oludayo Tade, Collins Obinna Udechukwu, Minou Ella Mebane, Donata Francescato, Antonio Aiello, Dharma Satrya HD, Faruk Faruk, Pujiharto Pujiharto, Dhastagir Sultan Sheriff, Kwadwo Adusei-Asante, Darlene Ndasi, Veronica L. Irvin, Benmei Liu, Richard Lee, Nancy Breen

( ) The Editor(s) and the Author(s) 2021

The rights of the editor(s) and the author(s) have been asserted in accordance with the Copyright, Designs and Patents Act 1988. All rights to the book as a whole are reserved by INTECHOPEN LIMITED. The book as a whole (compilation) cannot be reproduced, distributed or used for commercial or non-commercial purposes without INTECHOPEN LIMITED's written permission. Enquiries concerning the use of the book should be directed to INTECHOPEN LIMITED rights and permissions department (permissions@intechopen.com).

Violations are liable to prosecution under the governing Copyright Law .

\section{(cc) BY}

Individual chapters of this publication are distributed under the terms of the Creative Commons Attribution 3.๑ Unported License which permits commercial use, distribution and reproduction of the individual chapters, provided the original author(s) and source publication are appropriately acknowledged. If so indicated, certain images may not be included under the Creative Commons license. In such cases users will need to obtain permission from the license holder to reproduce the material. More details and guidelines concerning content reuse and adaptation can be found at http : //www . intechopen . com/copyright-policy. html .

Notice

Statements and opinions expressed in the chapters are these of the individual contributors and not necessarily those of the editors or publisher. No responsibility is accepted for the accuracy of information contained in the published chapters. The publisher assumes no responsibility for any damage or injury to persons or property arising out of the use of any materials, instructions, methods or ideas contained in the book.

First published in London, United Kingdom, 2021 by IntechOpen

IntechOpen is the global imprint of INTECHOPEN LIMITED, registered in England and Wales, registration number: 11086078 , 5 Princes Gate Court, London, SW7 2QJ, United Kingdom Printed in Croatia

British Library Cataloguing-in-Publication Data

A catalogue record for this book is available from the British Library

Additional hard and PDF copies can be obtained from orders@intechopen . com

Psycho-Social Aspects of Human Sexuality and Ethics

Edited by Dhastagir Sultan Sheriff

p. $\mathrm{cm}$.

Print ISBN 978-1-83962-441-4

Online ISBN 978-1-83962-442-1

eBook (PDF) ISBN 978-1-83962-443-8 


\section{We are IntechOpen, \\ the world's leading publisher of Open Access books}

Built by scientists, for scientists

\section{$5,200+$}

Open access books available

156

Countries delivered to
$127,000+$

International authors and editors
$150 \mathrm{M}+$

Downloads

Our authors are among the

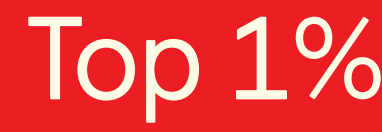

most cited scientists

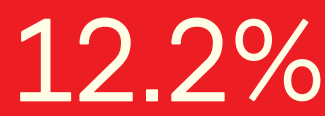

Contributors from top 500 universities

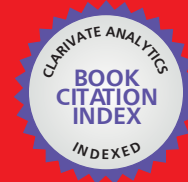

WEB OF SCIENCE ${ }^{\mathrm{TM}}$

Selection of our books indexed in the Book Citation Index in Web of Science ${ }^{\mathrm{TM}}$ Core Collection (BKCI)

Interested in publishing with us?

Contact book.department@intechopen.com

Numbers displayed above are based on latest data collected.

For more information visit www.intechopen.com

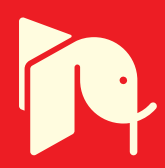





\section{Meet the editor}

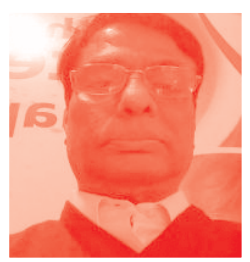

Dhastagir Sultan Sheriff is a life member of the European Society for Human Reproduction and Early Human Development, Association of Physiologists and Pharmacologists of India, member of the National Academy of Medical Sciences, New Delhi, and resource person for UNESCO for Medical and Bioethics. Dr. Sheriff has authored five books including a textbook on medical biochemistry with additional interest in human sexology. He had editorials written in the British Journal of Sexology, Journal of Royal Society of Medicine, Postgraduate Medicine, and Scientist. He was a former Rotarian, Citizen Ambassador, and was selected for the Ford Foundation Fellowship. 



\section{Contents}

Introductory Chapter: Sex, Sexuality and Ethics - An Indian Perspective by Dhastagir Sultan Sheriff

Chapter 2

Adolescents and Young Adults: Targeting the Unique Challenges of This High Risk Group by Kari Schneider, Joanna Ekstrom and Rachel Cafferty

Chapter 3

Characterizing Rapists and Their Victims in Select Nigeria Newspapers by Oludayo Tade and Collins Udechukwu

Chapter 4

Experiences of Sexual and Reproductive Healthcare Professionals Working with Migrant Women Living with Female Genital Cutting in Western Australia by Darlene Ndasi and Kwadwo Adusei-Asante

Chapter 5

Don't Objectify Me!: Sexual Self-Monitoring, Coping, and Psychological Maladjustment

by Catherine Baggett, Craig Nagoshi and Julie Nagoshi

Chapter 6

Political Gender Gap and Social Dominance Orientation

by Minou Ella Mebane, Antonio Aiello and Donata Francescato

Chapter 7

Psychological Health Influences of Legal-Marriage and -Partnerships on Same-Sex Couples

by William N. Elwood, Veronica L. Irvin, Benmei Liu, Richard Lee and Nancy Breen

Chapter 8

"Kidnapping the Bride"-A Traditional Sasak Wedding Seen in Sesak Cinta Di Tanah Sasak Novel: A Model in Contemporary Indonesian Literature Studies

by H.D. Dharma Satrya, Faruk Faruk and Pujiharto Pujiharto 



\section{Preface}

Sexual ethics deals with the choices one makes regarding sexual practice and preferences. With the emerging newer trends in sexuality and clashes with the traditional practice of human sexuality, there is a need for introspection and a framework of ethical principles that may guide human sexuality and its practice. Human sexuality is more than a biological phenomenon. It is a living experience that makes one understand how one views his or her personality and body. The majority of the time, health professionals spend time treating the patient rather than the patient's sexuality. The growing number of rapes and rape victims, sexual harassment cases and the violation of the right to have or resist sex in a marriage, the need to get consent before sex, and the trend of "living together" in corporate cities necessitate an introspection during a pandemic. Genital mutilation and its impact on the psychology of women need special attention. Many health professionals feel uncomfortable discussing sexuality with the patient due to cultural issues as well as lack of information regarding human sexuality in dealing with such issues and patients.

In short, sex is less concrete. Open discussions about sexual identity and sexual practices will help people better understand themselves, others, and the world around them. This book gives a panoramic view of certain aspects of human sexuality in health and disease.

Dhastagir Sultan Sheriff Faculty of Medicine, Benghazi University, Benghazi, Libya 



\title{
Introductory Chapter: Sex, Sexuality and Ethics - An Indian Perspective
}

\author{
Dhastagir Sultan Sheriff
}

\begin{abstract}
"Ethics is not neutral information. Ethics is active formation," Jordan wrote in an email. "So an essential part of teaching sexual ethics is getting people to reflect honestly both on what they believe and on how they have been led to those beliefs." Jonathan G. Adler, 2016.
\end{abstract}

\section{Introduction}

It is said that "In the beginning, when Twashtri came to the creation of woman, he found that he had exhausted his materials in the making of man and that no solid elements were left.

In this dilemma, after profound meditation, he did as follows:

He took the rotundity of the moon, the twinkling of the stars, the curves of creepers, the clinging of tendrils, the trembling of grass, the slenderness of the reed, the bloom of flowers, the lightness of leaves, the tapering of the elephant's trunk, the glances of deer, the clustering of rows of bees, the joyous gaiety of sunbeams, the weeping of clouds, the fickleness of wind, the timidity of the hare, the vanity of the peacock, the softness of the parrot's bosom, the hardness of a diamond, the sweetness of honey, the cruelty of the tiger, the warm glow of the fire, the coldness of snow, the chattering of jays, the cooing of the kokila, the hypocrisy of the Crane, the fidelity of the Charvaka, compounding all these together, he made woman and gave her to man. But in 2 weeks, the man came crying: "O Mighty Waster of Mysteries! Thou who has made all the wonders of the world, take again the woman that thou gave it me; she teases^ tantalizes and tires me, and I cannot live with her anymore." And Twashtri took the woman away. But in 2 weeks, the man came again and cried out: "Give me back the woman" that thou made; I cannot live without her.

This ancient description of creation of woman and man's dependence on woman for a complete life speaks the essence of bonding between the two describing the bond as social, scientific, and sexual in nature [1].

\subsection{Marriage and sex}

The institution of marriage in Indian culture and ambience emphasizes that "Sexual act has to be after marriage." This sets the moral principle for ethical evaluation. Ethics in simpler terms describes choice as an individual right but within the confines of social structure where we live in [2].

The word "sex" itself is considered to be discussed with responsibility and in private. Sexuality and sex are considered to be a part of one's personal domain 
whose sanctity cannot be transgressed. In other words, sex is viewed not just as a biological instinct but as an essential human activity and living.

One cannot discuss ethics without dissecting sex out of human sexuality as a biological need and instinct.

- It is a complex phenomenon that reflects our personality.

- According to WHO (2002), sexuality is "A central aspect of being human throughout life and it encompasses sex, gender identities and roles, sexual orientation, and social well-being that relates to one's sexuality."

- It can be influenced by biological, psychological, sociocultural, and religious factors [3].

\subsection{Science of sex and human sexual response cycle}

When one discusses sex, it is assumed that the person or the community have the right kind of knowledge both scientific and social. The science of sex and the human sexual response cycle has to become an essential part of analyses.

Human sexual response cycle is broadly divided into desire (libido), arousal (excitement), orgasm, and resolution [4].

Sexual desire or libido is shaped by age, sex, past experiences, values, social norms, and culture. It is felt that a woman cannot openly reveal her desire and it has to be always the male who has to initiate the cycle. Any open exposition of desire by the woman is considered as a taboo and even talking about sex is considered as a bad virtue. The verbal, visual, auditory, or touch stimuli trigger such a desire. It is required that sexual activities have to be private and personal.

\subsection{Survey regarding sexuality}

A survey to test the knowledge about sexual response cycle and its physiological effects were carried out over a period of 5 years. The number of respondents or participants was around 4400 persons of either sex [5].

The percentage of people who did not know about the sexual response cycle was $55 \%$ of women and $45 \%$ of men were ignorant. The rest of the people had no true information about the other three stages of the cycle. The urban people seemed to have better awareness compared to rural population. Rather, it was difficult to get the rural population to respond to the questionnaire, which required lots of persuasion and help from the village chief. They responded with total ignorance of these different phases. The rural population formed $49 \%$ of the total cases screened. The rest were from urban areas (51\%). There was also significant difference between the perception of sex between the urban and rural cases. Majority of the rural population answered that the very purpose of having sex is for procreation ( $80 \%$ of the rural population) and the rest of the rural population did not wish to respond to the question about having sex with the partner. Some of the urban and rural population did not have adequate knowledge about orgasm. The response did bring out a fact that most of the time the needs of female partner is not respected or given serious thought. Many did not know the difference between sex and gender. It has to be explained that sex is related to chromosome, hormones, and differences in the anatomy of the sex organs. Gender refers to the attitudes, interests, behaviors, and activities. Rather very few could understand that sex is biological instinct and gender is related to social structure [6].

Many did not understand the development of sexuo-erotic orientation that one could be heterosexual or homosexual or transgender. It was difficult to 
explain how one develops sexual orientation. It has to be taught that sexuoorientation starts with initial exposure to hormonal influences along with environment where an individual develops or lives. This scientific explanation or knowledge very few had and could not believe or understand how one behaves differently to different perceptions of sexuality. Rather, though there was a tradition of child marriage or the description of sex in the pages of Kamasutra, many people do not yet understand or acknowledge forms of sexuality other than heterosexual relationship [7].

\subsection{Sexual rights}

"Sexual rights refer to the human rights of all persons with respect to the free and responsible expression of their sexuality and their control over their bodies. They encompass the right to experience a pleasurable sexuality, which is essential in and of itself and, at the same time, is a fundamental vehicle of communication and love between people. Sexual rights include the right to liberty and autonomy in the responsible exercise of sexuality" [8].

Sexual rights are human rights demanding equal rights, respect, consent, and shared responsibilities between consenting sexual partners irrespective of all other considerations including sexuo-erotic orientation.

What is the state of women when it comes choosing to a sexual partner. Every one of legal age has the right to marry and form a family. This choice cannot be extended to all societies for one has to consider the culture and social determinants of the institution of marriage and social structure. In Hindu joint family system, marriage becomes essential for exercising sexual rights. The usual custom is if one desires to marry the bride, the groom's family visits the bride's family to ask for the hand of the bride. Sometimes it may be one of the many visits of bridegroom to be in choosing a bride for his marriage. During the formal enquiry, the two families converse with each other and decide whether marriage between the two is possible including matching the horoscope of the two individuals to be married. Even if both partners love each other, they want to marry with the consent of the parents of both parties. There are so many customs one has to follow to finalize a relationship with marriage. This decision is influenced by the community they belong to, the social status of the family, and also the caste system. If the consenting adults live in a relationship say with premarital sex and they wish to become life partners, they still need to go through the religious customs of the family they belong to. This living together relationship, though prevalent in many of the metro cities, has not taken deep roots to infiltrate the age-old tradition of the families living in many rural areas. There are many social constraints that influence such relationships. It is not easy to get an accommodation for a living together couple unless they show evidence of their marriage. There is still honor killing when two consenting adults of different castes get married or when the two persons belong to two different religions. The right to choose one's partner, though it resides with the consenting adults, has to pass through many social hurdles to accomplish it $[8,9]$.

The right to seek pleasure after marriage is mutual and depends upon the foundation of that relationship. Though condoms are available freely in the market, its use is not universal for it creates a doubt in the mind of the partner why condom is used. This is not because of any social pressure but because they lack sex education. Slowly, this trend has taken a marked change through family planning and the AIDS awareness program. Even this contraceptive use for safe sex is usually forced on the woman compared to men. Seeking sexual pleasure requires an understanding of the sexual response cycle as well as the methods of sexual intercourse. Whether a woman had orgasm is not being expressed by 
the women for the fear being called as sexually aggressive. The consent to have sex is usually dominated by the male without giving due respect to the consent of the woman. The social strata of society play a bigger role where economically emancipated women demand equal right to have sex with mutual consent. Forcing a wife to have sex without her consent is considered as rape legally [10].

"Nowadays, parents simply suggest the person they feel is suitable for their son or daughter. Only if their child approves (after interacting with him or her), do things move ahead. Also, children are now increasingly taking the initiative to find their own partners. The number of people putting up their profiles at matrimonial sites is a case in point. So, children are now 'arranging' their own marriages," says Sanjeev Sharma, 29, a software engineer currently in the "marriage market," looking for a bride [11].

\subsection{Conclusion}

In India, sex and sexuality had undergone a vast transformation from the days of polygamy to living together life style. In short, sex generates fascinating debates. With roots in biology, the topic of sexuality" remains complex. Its pleasures are intoxicating and invigorating. The desire to have sex, its expression and experience differ between men and women [12].

Its discussion and social implications form the basis of ethical discussion and, therefore, an introspection of these issues from different perspectives is important and the book deals with some of these issues in a concise and scientific way.

\section{Author details}

Dhastagir Sultan Sheriff

Faculty of Medicine, Benghazi University, Benghazi, Libya

*Address all correspondence to: drdsheriff@gmail.com

IntechOpen

(C) 2021 The Author(s). Licensee IntechOpen. This chapter is distributed under the terms of the Creative Commons Attribution License (http://creativecommons.org/licenses/ by/3.0), which permits unrestricted use, distribution, and reproduction in any medium, provided the original work is properly cited. (cc) BY 


\section{References}

[1] Sanskrit Story of the Creation of woman. Trove. 11 August 1921

[2] WHO. Defining sexual health Report of a technical consultation on sexual health. Geneva: WHO; 2006, 28-31 January 2002. Available from: http:// www.who.int/reproductivehealth/ publications/sexual_health/defining_ sexual_health.pdf

[3] Knipe DM. Hinduism New York. San Francisco, London: Harper and Collins; 1991

[4] World Association for Sexual Health. Declaration of Sexual Rights. Adopted at the 14th World Congress of Sexology; 1999

[5] Masters WH, Johnson V. Human Sexual Response. 1st ed. Boston: Little, Brown Company; 1966

[6] Sheriff DS. Understanding Human Sexuality. Salem, India: Sri Ram Press; 2000

[7] Levin RJ. The Human Sexual Response Cycle. In: Wequih W, Ishak, editors. The Textbook of Clinical Sexual Medicine. AG: Springer International Publishing; 2017. pp. 39-551

[8] Burton R, Arbuthnet FF. Translated "The Kamasutra of Vatsyana". New York: Putnam Press; 1984. p 223

[9] Kaustav C, Rajashri GT. Indian concepts of sexuality. Indian Journal of Psychiatry. 2013;55:5250-5255

[10] Mayur G. The Evolution of Hindu Marriage. Trends through Indian History. TWB Ideas Wedding Brigades

[11] Richa Pant/A modern guide to arranged marriage. 1 November 2006. Available from: rediff.NEWS
[12] Oriel J. Sexual pleasure as a human right: Harmful or helpful to women in the context of HIV/AIDS? Women's Studies International Forum. 2005;28:3920404 



\title{
Adolescents and Young Adults: Targeting the Unique Challenges of This High Risk Group
}

Kari Schneider, Joanna Ekstrom and Rachel Cafferty

\begin{abstract}
Adolescents, particularly sexual minority youth, account for a disproportionate number of sexually transmitted infections and pelvic inflammatory disease cases. This population is at increased risk of infection and presents unique challenges when it comes to screening and treatment. This chapter will discuss these challenges and suggest some evidence based solutions. Challenges to be discussed include lack of physician comfort in discussing sexual activity with adolescents, issues of confidentiality, difficulties with contacting adolescent patients with their test results, and arranging for treatment of both patients and their partners. A discussion about expedited partner therapy will be included - the legal implications and the role it plays in sexually transmitted infection reduction efforts.
\end{abstract}

Keywords: adolescent, young adult, confidentiality, emergency department, LGBT, expedited partner therapy

\section{Introduction}

Adolescents and young adults 15-24 years of age represent approximately $25 \%$ of the sexually active United States (US) population, but account for nearly half of all new sexually transmitted infection (STI) cases [1]. Adolescents have the highest incidence of Neisseria gonorrhea and Chlamydia trachomatis among any sexually active age group [2]. The most significant complication from STIs is pelvic inflammatory disease (PID) with approximately 200,000 adolescents diagnosed annually in the US [3]. This population also has an elevated risk of subsequent STIs after initial PID, thus putting them at increased risk of associated reproductive health sequelae such as infertility, ectopic pregnancy, and chronic abdominal pain $[4,5]$. Given that adolescents are just entering their reproductive years, STI and PID reduction efforts must be maximized.

When it comes to STI reduction efforts in adolescents, a multifactorial approach is necessary. It starts with meeting adolescents where they intersect with health care professionals (this is not always simply in a physician's office setting), talking to adolescents about sex in a confidential manner, screening appropriately, notifying patients with positive results, and treating both the patients and their partners to prevent reinfection. This chapter discusses these aspects of tackling STI reduction in this age group. 


\section{Inherent risk of the adolescent period}

Adolescence is a period of rapid physical, social-emotional, and sexual change, characterized by experimentation and exploration as one searches to define their own identity. For many, sexual debut occurs during adolescence. A number of behavioral, biological, and cultural factors among sexually active adolescents and young adults lead to higher risk of acquiring an STI in this age group. Biologically, young women are more susceptible to STIs because of increased cervical ectopy (which refers to columnar cells, usually located within the cervical canal, being located on the outer surface of the cervix). These areas of ectopy are fragile with thin, vascularized epithelium and thus blood vessels lie in close contact with the vaginal environment; possibly diminishing mucosal barriers to sexually transmitted infections.

Beyond biological factors, adolescents are more likely to engage in unprotected sex, have multiple sexual partners, and use drugs and alcohol, which may result in high risk sexual behaviors [6].

According to the Centers for Disease Control and Prevention (CDC), condom use, as reported by sexually active high school students, is inconsistent. Among US high school students surveyed in 2017, 46\% did not use a condom the last time they had sex [6]. Young women are using very effective and long-lasting contraceptive options like intrauterine devices and implants at higher rates and should be applauded for this, but these offer no protection against STIs such as gonorrhea or chlamydia [6].

Sexual minority youth, identified as lesbian, gay, bisexual, transgender (LGBT), represent a subset of the adolescent population at heightened risk for sexually transmitted infections. While many LGBT youth are resilient and thriving, the effects of homophobia, heterosexism, and parental rejection may result in psychological distress and a subsequent increase in self-destructive risk behaviors [7]. Sexual minority youth are more likely to report having intercourse, initiating intercourse at younger ages (before age 13), have a greater number of sexual partners $(\geq 4$ partners), and are less likely to use barrier contraception compared to heterosexual or cis-gender peers [7]. LGBT youth have higher rates of homelessness, which results in increased sexual violence and survival sex [7]. Transgender teens (particularly male to female transgender youth) have higher rates of HIV and STIs and selfreport lower rates of preventive health checkups and overall poorer health $[7,8]$.

\section{Utilization of health care by adolescents}

Utilization of health care by adolescents (LGBT, heterosexual, and cis-gender alike) is complicated, and their overall usage of health care is low. Higher rates of STIs among adolescents may reflect barriers to accessing preventive care and services for sexual and reproductive health. The American Academy of Pediatrics (AAP) recommends at least one preventive health visit per year. The data, however, show that only $40-80 \%$ of adolescents report a primary care visit within 12 months [9-11]. A 2010 study of insured adolescents, revealed that one-third had no preventive care visits between the ages of 13 through 17 years, and another $40 \%$ had only one preventive care visit during this 4 year period of their lives [12]. Barriers in accessing care include believing they only need appointments when sick, lack of transportation, conflict between school and clinic hours, concerns about confidentiality, lack of health insurance, inability to pay for contraception or STI testing, and stigma surrounding accessing STI services [10].

While non-preventive care visits are slightly more frequent among adolescents (approximately 1-1.5 visits per year among adolescents age 11 through 17 years), a busy practice environment and short encounters with a clinician may not afford 
the opportunity for full discussions of sexual health, risk taking behaviors, and concerns outside of the presenting problem [12]. Nordin and colleagues recommend a no-missed-opportunities paradigm, by which all adolescent visits, regardless of busy practice environments and short encounters with a clinician, be viewed as an opportunity to provide preventive care services [12]. One clinic successfully increased the number of preventive health visits for adolescents by "flipping" acute/ sick visits into well-care visits when patients were overdue [13]. Given the disproportionate burden of STIs among adolescents, preventive visits including discussions about sexuality and sexual risk factors are of paramount importance.

The AAP, American Medical Association, and Society for Adolescent Medicine recommend that physicians discuss sexuality with youth as part of routine healthcare. Physicians have an important role in helping adolescents develop healthy relationships and behaviors. However, when adolescents intersect with healthcare, physicians may not feel fully equipped or have the expertise in managing specific sexual health needs. The literature suggests that primary care pediatricians are not consistent in having important discussions regarding sexuality and sexual risktaking behaviors with adolescents [14]. In a survey of AAP members who provide health supervision visits to adolescent patients, $58 \%$ of pediatricians self-reported a lack of interest in adolescent health issues [14]. Perhaps more alarming is the fact that $<9 \%$ of those surveyed were very familiar with AAP policies or CDC recommendations regarding STI and HIV testing for youth [14]. Additionally, 25\% of providers did not know their own state laws regarding testing of teens for STIs without parental consent [14]. While pediatricians believed that reproductive health services were an important part of adolescent health care delivery, less than half $(46 \%)$ recommend STI testing for all sexually active teenagers and the vast majority $(>70 \%)$ did not prescribe or distribute condoms, or provide education on effective condom use [14].

Given that adolescents are not always consistent with seeing their primary physicians for preventive visits, providers need to utilize other opportunities for screening for STIs. School-based health centers (SBHCs) are another important place where adolescents may access health care. SBHCs remove some of the common barriers to health care for this age group, including scheduling and transportation, as the clinics are located where the adolescents already spend their days in school. Many of these centers are in urban areas, and they primarily serve high schools, alternative schools, or schools with a combination of grade levels [15]. Adolescents who use SBHCs have been shown to have more primary care visits and fewer emergency department visits than those who do not use these clinics. Although SBHCs are sometimes prohibited from dispensing contraceptives by school district policy or state law, some are able to provide these services, and they also can screen for and treat STIs.

Adolescents also seek care in emergency departments (EDs). The ED has been described as a critical "safety net", treating patients without other sources of care [16]. Adolescents make up about $15 \%$ of the patient population in the emergency department $[17,18]$. One study found that $18 \%$ of $10-17$-year-olds, and $25 \%$ of 18-24-year-olds, had visited an emergency department in the previous year [19]. And the rates of STIs found for this patient population in this venue are significant [20-23]. PID is the most common diagnosis among adolescents seeking care for STIs in US EDs and studies have unfortunately shown incorrect treatment of PID in this setting [24, 25].

While the ED is a readily-available place for adolescents to receive health care, it poses many challenges, especially given the complex nature of adolescent health care. Goyal et al. demonstrated that, in adolescents presenting to the ED with genitourinary complaints, the prevalence of STIs was 26\% [21]. Schneider et al. 
found that in adolescents presenting to the ED with non-genitourinary complaints, the prevalence of STIs was 10\% [22]. Given the nature of emergency care and lack of continuity, there is concern that patients testing positive but were not treated in the ED may become lost to follow up, and therefore remain untreated [26]. This needs to be balanced with antibiotic stewardship and development of antibiotic resistant organisms associated with overtreatment [27]. This highlights some of the complexities of adolescent STI reduction initiatives in the ED setting.

\section{Confidentiality}

Where ever they choose to seek care, confidentiality is important to adolescents. Multiple medical and legal organizations recognize the need for providing confidential health care to adolescents and a growing body of research has shown the importance of this, but this comes with challenges [28-30]. According to the Society for Adolescent Medicine, "Confidentiality protection is an essential component of health care for adolescents because it is consistent with their development of maturity and autonomy and without it, some adolescents will forgo care" [28]. A 1997 study by Ford et al. showed that assurances of confidentiality increased the number of adolescents willing to return for a future visit to a physician's office by 10 percentage points, from 62 to $72 \%(P=0.001)$ [29]. Additionally, adolescents who report health risk behaviors have been shown to have an increased likelihood of citing confidentiality concerns as a reason for forgone health care [30].

In the United States, each state has legal statues that authorize minors to consent for care under a variety of circumstances [31]. Care that minors are allowed to consent for without a parent usually includes contraceptive services, pregnancy-related care, diagnosis and treatment of STIs, care related to a sexual assault, treatment for drug or alcohol problems, or mental health services. Some states, however, require that a minor be of a certain age (generally around 14 years old) before being allowed to consent [32].

The Health Insurance Portability and Accountability Act (HIPAA) Privacy Rule creates rights for individuals to have access to their protected health information and to control the disclosure of that information in some circumstances. It contains specific requirements pertaining to the medical records of minors [33]. The HIPAA Privacy Rule provides that, in general, when minors legally consent to health care or can receive it without parental consent, the parent does not necessarily have the right to access the minor's health information. This is all dependent, however, on each individual state's laws. Thus, a health care provider must look to state law to determine whether it specifically addresses the confidentiality of a minor's health information. If state law is silent on the question of parents' access, a health care professional exercising professional judgment has discretion to determine whether or not to grant access [33].

Likely one of the biggest limitations in providing confidential care to adolescents has to do with payment. Most often, parents or parents' health insurance pays for an adolescent's health care. As of the time of publication of this text, there is no legal way to prevent a parent from viewing a billing statement and/or explanation of benefits (EOBs) from the insurance company. EOBs are notifications to policyholders that health care services were provided under a health insurance plan, including those services provided to any dependents covered by the plan. EOBs generally disclose the name and of the provider and the specific laboratory tests used or other services rendered. They are intended to protect policyholders and insurers from fraud and abuse and to explain financial obligations, but can have unanticipated and unintended negative consequences such as a breach of confidentiality [34]. 


\section{Testing and follow-up}

Testing and follow-up are also areas with unique nuances when it comes to adolescent patients. Methods for testing and/or screening adolescents for gonorrhea and chlamydia include self-obtained vaginal swabs, self-collected urine samples, and provider-collected endocervical swabs. For adolescents, the idea of a provider-collected specimen can be a barrier to seeking care [35, 36]. Self-collected specimens have been shown time and again in the medical literature to be preferred by adolescents over provider-collected specimens [37]. Many studies have also evaluated the utility of these other forms of testing and have found that the sensitivity and specificity of self-collected swabs and urine samples compared to swabs collected by clinicians supports the use of these tests in screening for gonorrhea and chlamydia [38, 39].

Given that adolescents do not always present regularly for preventive health care visits, the acceptability of and ease of collection with self-collected specimens may allow clinicians an opportunity to screen patients in the clinic for STIs who are not presenting for pelvic or urogenital examinations and might not otherwise be screened as regularly as they should be.

Follow up of adolescents can be problematic, especially for those seeking care in emergency departments. ED personnel in one study cited difficulty in reaching adolescents and the ease of empiric treatment to justify the practice of empirically treating STI tested patients and only providing follow-up contact to those who tested positive but were not treated at the ED visit [40]. Confirming that STI-positive patients receive appropriate treatment is a vital component of any screening initiative. Success in contacting adolescents with their results has been found, especially when a confidential cell phone number is used [41, 42]. Reed et al. worked on increasing the proportion of adolescent patients able to be contacted with their test results from 45 to $65 \%$ and decreased their lost to follow up rate [43]. This was done almost entirely by focusing on making sure that a confidential phone number was documented in the electronic medical record. They also gave out a card with contact information for a cell phone given to a nurse practitioner who was dedicated to contacting and being available for being contacted by the patients regarding their results. They learned that adolescents often have cell phone plans with limited minutes of talk time, but can still send and receive texts. So while they might not answer their phones or check their messages frequently, they will respond quickly to a text message.

It is important for adolescents to be aware of their test results, even in the case where they have already been empirically treated, as having knowledge about their diagnosis can lead to behavior changes [44]. Dr. Reed's group also followed up, by phone, a convenience sample of adolescent women who were empirically treated in the ED or teen clinic setting for STI [45]. They found that those who believed they had an STI were more likely to abstain from sexual activity and to notify their partners. Those who were treated with antibiotics but did not believe they tested positive for STIs did not change their behavior.

\section{Treatment of adolescents patients and their partners}

Treating the partners of STI-positive patients is another important factor in STI reduction. Ideally, partners of STI-positive patients would seek health care for evaluation, treatment, and counseling in person. This can prove to be a challenge in all ages, especially in adolescents and unfortunately, rates of reinfection in adolescents and young adults are high. A 2008 study by Gaydos et al. reported up to $26 \%$ of 
adolescent and young adult women were reinfected with their STI within 12 months [46]. The AAP has endorsed a position paper by the Society for Adolescent Health and Medicine supporting the use of expedited partner therapy (EPT) as a treatment option for heterosexual sex partners of adolescents with gonorrhea and chlamydia when other partner treatment methods are impractical or unsuccessful [47].

EPT is a management technique in which medications or prescriptions are provided for the partner of a patient who tests positive for gonorrhea and/or chlamydia without physical examination of that partner. It involves prescribing the medication (it is acceptable to write "Expedited Partner Therapy" in place of the partner's name; no date of birth needed). It should also include treatment instructions, warnings about the medications, general health counseling, and a statement that advises the partner to seek medical evaluation in the setting of symptoms.

This technique has proven to be beneficial [48-50]. In a 2005 study by Golden et al., EPT was more effective than standard referral of partners in reducing persistent or recurrent infection among patients with gonorrhea ( $3 \%$ vs. $11 \%, \mathrm{P}=0.01)$ [50]. It is currently not recommended, however, for men who have sex with men or women with trichomoniasis because of increased risk of coinfections and lack of supporting evidence in these populations.

Optimal STI control requires more than testing and treatment to disrupt transmission; the CDC urges us to counsel these patients on partner treatment and safer sex practices. In adolescents especially, communication is key. Talking with adolescents about their risks of reinfection, both surrounding their acute infection as well as in the future unless they change their behaviors, is of paramount importance.

\section{Conclusion}

Adolescents, particularly sexual minority youth, account for a disproportionate number of sexually transmitted infections. Several factors that put adolescents at increased risk for STIs have been reviewed here, including high-risk behaviors (unprotected sex, sex with multiple partners, increased substance abuse, survival sex by homeless LGBT youth) and decreased reproductive and sexual health care utilization by teens. Barriers in accessing preventive care and treatment for STIs contributes to higher STI rates in this vulnerable population. Despite recommendations to discuss sexuality and risk behaviors with adolescents, providers may lack time, resources and appropriate knowledge surrounding screening guidelines, minor consent, and confidentiality to fully meet the needs of adolescent patients. A strong STI reduction initiative must place emphasis on the adolescent population and its unique attributes and needs. 


\section{Author details}

Kari Schneider ${ }^{1 *}$, Joanna Ekstrom ${ }^{1}$ and Rachel Cafferty ${ }^{2}$

1 University of Minnesota Masonic Children’s Hospital, Minneapolis, Minnesota, USA

2 Children’s Hospital Colorado, Aurora, Colorado, USA

*Address all correspondence to: sch1005@umn.edu

\section{IntechOpen}

(C) 2019 The Author(s). Licensee IntechOpen. This chapter is distributed under the terms of the Creative Commons Attribution License (http://creativecommons.org/licenses/ by/3.0), which permits unrestricted use, distribution, and reproduction in any medium, provided the original work is properly cited. (cc) BY 


\section{References}

[1] Centers for Disease Control and Prevention. Sexually Transmitted Diseases: Adolescents and Young Adults. 7 Dec 2017. Available from: https://www. cdc.gov/std/life-stages-populations/ adolescents-youngadults.htm

[2] Centers for Disease Control and prevention. Sexually Transmitted Disease Surveillance 2017: Gonorrhea. Available from: https://www.cdc.gov/ std/stats17/gonorrhea.htm

[3] Sutton MY, Sternberg M, Zaidi A, St Louis ME, Markowitz LE. Trends in pelvic inflammatory disease hospital discharges and ambulatory visits, United States, 1985-2001. Sexually Transmitted Diseases. 2005;32(12):778-784

[4] Westrom L, Joesoef R, Reynolds G, Hagdu A, Thompson SE. Pelvic inflammatory disease and fertility. A cohort study of 1,844 women with laparoscopically verified disease and 657 control women with normal laparoscopic results. Sexually Transmitted Diseases. 1992;19(4):185-192

[5] Trent M, Chung SE, Forrest L, Ellen JM. Subsequent sexually transmitted infection after outpatient treatment of pelvic inflammatory disease. Archives of Pediatrics \& Adolescent Medicine. 2008;162(11):1022-1025

[6] Centers for Disease Control and Prevention. Youth Risk Behavior Surveillance-United States. 2017. Available from: https://www.cdc. gov/mmwr/volumes/67/ss/ss6708a1. htm?s_cid=ss6708a1_w

[7] Levine DA, Committee on Adolescence. Office-based care for lesbian, gay, bisexual, transgender, and questioning youth. Pediatrics. 2013;132(1):e297-e313
[8] Rider GN, McMorris BJ, Gower AL, Coleman E, Eisenberg ME. Health and care utilization of transgender and gender nonconforming youth: A population-based study. Pediatrics. 2018;141(3):e20171683

[9] Sabharwal M, Masinter L, Weaver KN. Examining time to treatment and the role of school-based health centers in a school-based sexually transmitted infection program. The Journal of School Health. 2018;88(8):590-595

[10] Aalsma MC, Gilbert AL, Xiao S, Rickert VI. Parent and adolescent views on barriers to adolescent preventive health care utilization. The Journal of Pediatrics. 2016;169:140-145

[11] Adams SH, Park MJ, Irwin CE Jr. Adolescent and young adult preventive care: Comparing national survey rates. American Journal of Preventive Medicine. 2015;49(2):238-247

[12] Nordin JD, Solberg LI, Parker ED. Adolescent primary care visit patterns. Annals of Family Medicine. 2010;8(6):511-516

[13] Kumra T, Antani S, Johnson SB, Weaver SJ. Improving adolescent preventive care in an urban pediatric clinic: Capturing missed opportunities. The Journal of Adolescent Health. 2017;60(6):734-740

[14] Henry-Reid LM, O'Connor KG, Klein JD, Cooper E, Flynn P, Futterman DC. Current pediatrician practices in identifying high-risk behaviors of adolescents. Pediatrics. 2010;125(4):e741-e747

[15] Council On School Health. School-based health centers and pediatric practice. Pediatrics. 2012;129(2):387-393 
[16] Rhodes KV, Gordon JA, Lowe RA. Preventive care in the emergency department, part I: Clinical preventive services--are they relevant to emergency medicine? Society for Academic emergency medicine public health and education task force preventive services work group. Academic Emergency Medicine. 2000;7(9):1036-1041

[17] Ziv A, Boulet JR, Slap GB. Emergency department utilization by adolescents in the United States. Pediatrics. 1998;101(6):987-994

[18] Melzer-Lange M, Lye PS. Adolescent health care in a pediatric emergency department. Annals of Emergency Medicine. 1996;27(5):633-637

[19] Mulye TP, Park MJ, Nelson CD, Adams SH, Irwin CE Jr, Brindis CD. Trends in adolescent and young adult health in the United States. The Journal of Adolescent Health. 2009;45(1):8-24

[20] Anaene M, Soyemi K, Caskey $\mathrm{R}$. Factors associated with the overtreatment and under-treatment of gonorrhea and chlamydia in adolescents presenting to a public hospital emergency department. International Journal of Infectious Diseases. 2016;53:34-38

[21] Goyal MK, Teach SJ, Badolato GM, Trent M, Chamberlain JM. Universal screening for sexually transmitted infections among asymptomatic adolescents in an urban emergency department: High acceptance but Low prevalence of infection. The Journal of Pediatrics. 2016;171:128-132

[22] Schneider K, FitzGerald M, Byczkowski T, Reed J. Screening for asymptomatic gonorrhea and chlamydia in the pediatric emergency department. Sexually Transmitted Diseases. 2016;43(4):209-215

[23] Silva A, Glick NR, Lyss SB, Hutchinson AB, Gift TL, Pealer LN, et al. Implementing an HIV and sexually transmitted disease screening program in an emergency department. Annals of Emergency Medicine. 2007;49(5):564-572

[24] Goyal M, Hersh A, Luan X, Localio R, Trent M, Zaoutis T. National trends in pelvic inflammatory disease among adolescents in the emergency department. The Journal of Adolescent Health. 2013;53(2):249-252

[25] Solomon M, Tuchman L, Hayes K, Badolato G, Goyal MK. Pelvic inflammatory disease in a pediatric emergency department: Epidemiology and treatment. Pediatric Emergency Care. 2017. DOI: 10.1097/ PEC.0000000000001148

[26] Krivochenitser R, Bicker E, Whalen D, Gardiner C, Jones JS. Adolescent women with sexually transmitted infections: Who gets lost to follow-up? The Journal of Emergency Medicine. 2014;47(5):507-512

[27] Bolan GA, Sparling PF, Wasserheit JN. The emerging threat of untreatable gonococcal infection. The New England Journal of Medicine. 2012;366(6):485-487

[28] Ford C, English A, Sigman

G. Confidential health care for adolescents: Position paper for the society for adolescent medicine. The Journal of Adolescent Health. 2004;35(2):160-167

[29] Ford CA, Millstein SG, HalpernFelsher BL, Irwin CE Jr. Influence of physician confidentiality assurances on adolescents' willingness to disclose information and seek future health care. A randomized controlled trial. Journal of the American Medical Association. 1997;278(12):1029-1034

[30] Lehrer JA, Pantell R, Tebb K, Shafer MA. Forgone health care among U.S. adolescents: Associations between risk 
characteristics and confidentiality concern. The Journal of Adolescent Health. 2007;40(3):218-226

[31] Kenney AEK. State Minor Consent Laws. Chapel Hill, North Carolina: Center for Adolescent Health \& the Law; 2003

[32] Guttmacher Institute. State Laws and Policies: Minors' Access to STI Services. 1 May 2019. Available from: https://www. guttmacher.org/state-policy/explore/ minors-access-sti-services

[33] English A, Ford CA. The HIPAA privacy rule and adolescents: Legal questions and clinical challenges. Perspectives on Sexual and Reproductive Health. 2004;36(2):80-86

[34] Society for Adolescent $H$, Medicine, American Academy of P. Confidentiality protections for adolescents and young adults in the health care billing and insurance claims process. The Journal of Adolescent Health. 2016;58(3):374-377

[35] Avuvika E, Masese LN, Wanje G, Wanyonyi J, Nyaribo B, Omoni G, et al. Barriers and facilitators of screening for sexually transmitted infections in adolescent girls and young women in Mombasa, Kenya: A qualitative study. PLoS One. 2017;12(1):e0169388

[36] Tilson EC, Sanchez V, Ford CL, Smurzynski M, Leone PA, Fox KK, et al. Barriers to asymptomatic screening and other STD services for adolescents and young adults: Focus group discussions. BMC Public Health. 2004;4:21

[37] Serlin M, Shafer MA, Tebb K, Gyamfi AA, Moncada J, Schachter J, et al. What sexually transmitted disease screening method does the adolescent prefer? Adolescents' attitudes toward first-void urine, self-collected vaginal swab, and pelvic examination. Archives of Pediatrics \& Adolescent Medicine. 2002;156(6):588-591

[38] Fang J, Husman C, DeSilva L, Chang R, Peralta L. Evaluation of self-collected vaginal swab, first void urine, and endocervical swab specimens for the detection of Chlamydia trachomatis and Neisseria gonorrhoeae in adolescent females. Journal of Pediatric and Adolescent Gynecology. 2008;21(6):355-360

[39] Lunny C, Taylor D, Hoang L, Wong T, Gilbert M, Lester R, et al. Self-collected versus cliniciancollected sampling for chlamydia and gonorrhea screening: A systemic review and meta-analysis. PLoS One. 2015;10(7):e0132776

[40] Gillespie GL, Reed J, Holland CK, Munafo JK, Ekstrand R, Britto MT, et al. Pediatric emergency department provider perceptions of universal sexually transmitted infection screening. Advanced Emergency Nursing Journal. 2013;35(1):76-86

[41] Reed JL, Huppert JS, Taylor RG, Gillespie GL, Byczkowski TL, Kahn JA, et al. Improving sexually transmitted infection results notification via mobile phone technology. The Journal of Adolescent Health. 2014;55(5):690-697

[42] Maraynes ME, Chao JH, Agoritsas K, Sinert R, Zehtabchi S. Screening for asymptomatic chlamydia and gonorrhea in adolescent males in an urban pediatric emergency department. World Journal of Clinical Pediatrics. 2017;6(3):154-160

[43] Huppert JS, Reed JL, Munafo JK, Ekstrand R, Gillespie G, Holland $\mathrm{C}$, et al. Improving notification of sexually transmitted infections: A quality improvement project and planned experiment. Pediatrics. 2012;130(2):e415-e422 
[44] Reed JL, Zaidi MA, Woods

TD, Bates JR, Britto MT, Huppert

JS. Impact of post-visit contact on emergency department utilization for adolescent women with a sexually transmitted infection. Journal of Pediatric and Adolescent Gynecology. 2015;28(3):144-148

[45] Huppert JS, Taylor RG, St Cyr S, Hesse EA, Reed JL. Point-of-care testing improves accuracy of STI care in an emergency department. Sexually Transmitted Infections. 2013;89(6):489-494

[46] Gaydos CA, Wright C, Wood BJ, Waterfield G, Hobson S, Quinn TC. Chlamydia trachomatis reinfection rates among female adolescents seeking rescreening in school-based health centers. Sexually Transmitted Diseases. 2008;35(3):233-237

[47] Burstein GR, Eliscu A, Ford K, Hogben M, Chaffee T, Straub D, et al. Expedited partner therapy for adolescents diagnosed with chlamydia or gonorrhea: A position paper of the Society for Adolescent Medicine. The Journal of Adolescent Health. 2009;45(3):303-309

[48] Kissinger P, Mohammed H, Richardson-Alston G, Leichliter JS, Taylor SN, Martin DH, et al. Patientdelivered partner treatment for male urethritis: A randomized, controlled trial. Clinical Infectious Diseases. 2005;41(5):623-629

[49] Trelle S, Shang A, Nartey L, Cassell JA, Low N. Improved effectiveness of partner notification for patients with sexually transmitted infections: Systematic review. BMJ. 2007;334(7589):354

[50] Golden MR, Whittington WL, Handsfield HH, Hughes JP, Stamm WE, Hogben M, et al. Effect of expedited treatment of sex partners on recurrent or persistent gonorrhea or chlamydial infection. The New England Journal of Medicine. 2005;352(7):676-685 



\title{
Chapter 3
}

\section{Characterizing Rapists and Their Victims in Select Nigeria Newspapers}

\author{
Oludayo Tade and Collins Udechukwu
}

\begin{abstract}
While offender and victim characterization offers clues to devising preventive strategies and victim therapy, existing studies in Nigeria are yet to capture this empirically. Using two national dailies, about 331 rape cases have been analyzed. The rapists were found to be of the age group 18-55 years. The victims' age has been reported to be between 1 and 20 years. Rape victims were mostly females (>90\%) compared to males. Most of the rapists are labeled as familiar foes meaning, father, relatives, or neighbors. Third-party guardianship is mentioned as predatory.
\end{abstract}

Keywords: rape, Nigeria, victim, crime, offender

\section{Introduction}

Sexual violence, rape in particular against girls and women, is a sad reality in Nigeria in particular and some parts of Africa. Like other crimes that go unreported, rape is mostly unreported to formal agencies of social control in Nigeria partly due to trust gap, associated stigma against the victims, fear of re-victimization, cultural barriers, religious sentiments, and the powerlessness of the victims in pursuing justice. Nonetheless, the media continues to play key roles in tracking rape cases in Nigeria by foregrounding rape episodes in their reportage. Through their coverage of rape stories, the "private problem" as experienced by a victim is made "public trouble" by eliciting societal reactions from state and non-state actors. In other words, rape is not only reported as crime or social problem, but also as an item that questions current state of morality and calling for moral awakening and vigilance [1]. In understanding deviance and crime, characterization of the act (Rape), actors (who they are), social situation (context of the crime) and the definers (reactions of the law and the public) of such actions become important not only in appreciating the magnitude of the problem but in devising prevention strategies and victim therapy. In this paper, we analyze a corpus of newspaper coverage of rape in Nigeria. We sought answers to: how do print media characterize rape offenders and victims?

In Nigeria, the criminal and the penal codes view rape as a serious offense based on how they defined the crime. Thus rape is defined under Section 357 of the Nigerian criminal code which is applicable to the southern part of Nigeria as: 
of any kind or by fear of harm, or by means of false and fraudulent representation as to the nature of the act, or in The case of a married woman, by personating her husband is guilty of An offense which is called rape.

In the Northern part of Nigeria, rape is defined under Section 282 of the penal code as:

1. "A man is said to commit rape who...has sexual intercourse With a woman in any of the following circumstances: (a) against Her will; (b) obtained by putting her in fear of death or of hurt; (c) With her consent when the man knows that he is not her husband And that her consent is given because she believes that he is the Man to whom she is or believes herself to be lawfully married; (d) With or without her consent when she is under fourteen years Of age or of unsound mind."

2. "Sexual intercourse by a man with his own wife is not considered as rape, if she has attained to puberty."

The two definitions show that in Nigeria, the conception of rape is seen as being perpetuated only by men against women and not vice versa. In other places, this is not the case because it has also been recognized that a man can also be victim to rape and it may be perpetuated by any person including a woman or a man. The definitions also never factored in non-penetrative rape such as oral sex and use of objects or other instrument aside from the organs as rape. This puts into context what could be adjudicated upon in Nigeria owing to existing laws. Not minding Nigeria law, literature recognizes date rape, anger rape, prison rape, war rape, spousal rape, gang rape, sadistic rape, statutory rape, elderly rape, rape of children and rape by deception [2].

In Nigeria, Lagos state Police Command recorded 678 rape cases between March 2012 and March 2013 alone. Despite these statistics, few are convicted while many withdraw their cases [3]. Making up for the unrecorded and unreported are the media through their coverage of rape cases. A few examples of such will suffice here: a 55-year-old man was reported to have abducted and raped a 14-year-old girl for 4 days [4]. Also a pastor was reported to have raped his neighbors' daughter aged 12 after luring her with biscuits and money [5]. Equally, a 15 year old girl was also raped by a tricycle rider after which he gave her N200 [6]. Similarly, a teacher was arrested after he defiled his 3 year old student [7]. Also at least 100 cases of rape were also reported in court in Kano in 2013, in which only about 40 offenders were convicted. Similarly, in Anambra state, about 155 incidences and complaints on rape were recorded by some NGOs that worked on sexual violence in the state. However, only 12 out of the 155 cases were taken up by the police and surprisingly none of them was successfully prosecuted.

While rape statistics are crucial to showcase the enormity of rape, it nonetheless, does not capture the social reality of rape in those countries. This is because victims of rape or sexual violence as it is called elsewhere often do not report and therefore do not get captured in criminal statistics. A number of studies attest to the fact that police statistics never capture the magnitude of rape reality $[8,9]$. Furthermore, statistics are figures which do not capture narratives of perpetrators and victims. Existing data do not show such vital demographics which can be used in characterizing victims and their offenders. In other words, figures hide actualities and the "flesh" of the phenomenon needed for qualitative analysis. It is in this light that we add to existing body of researches on rape by filling this gap. 
Oduah [10] gave the following as factors that may lead to or be the cause of increase in rape cases in the Nigerian society, they are; gaps that are embedded in the laws of the country and the strict adherence by the law court for the provision of burden of proof by the victim and the prosecutors; delays in the judicial system; corruption of the judiciary; stigmatization; family ties (in situations where rape/ child rape was committed by people who are close relatives of the victims as a result the family settles the case among themselves and therefore not report these crimes to the police for prosecution); fear of attacks and intimidation (in the sense that the victims most times are afraid that if they report, the perpetrator or rapist may re-attack them and in some cases these rapists are deadly and cruel) immoral dressing on the part of the girls and inadequate parental care. It is however important to note that in a situation where the victim fails to provide the above proof of rape, the accused then has the privilege to walk free even if the act was indeed committed. As noted by Musbau [11] rape offenders are having a jolly ride because the burden of proof lies with the victim. The victim alone bears the burden of providing the bed sheets used to rape, providing her underwear and not washing herself before going to the police station or the hospital. Further challenge being faced by victims of rape includes delays in getting and providing the medical reports of the test. This is due to the poor state of health facilities and inadequate finance to quicken the processing of such medical reports.

Acts of rape is widespread elsewhere in Africa. For instance, rape is ubiquitous in South Africa (SA). In terms of statistics, SA recorded a total of 39,828 cases of rape (under the sexual offenses categorization of crime) between 2016 and 2017 [12]. In another African country, Zimbabwe, at least one woman is raped every 90 min while 500 women are sexually abused monthly. This means that at least 16 women get raped daily. Elsewhere in East Africa, Kenya recorded a total of 2648 rape cases as at 2015 [13].

Media construction of rape has produced different consequences in other climes. Nagar [14] stressed the factors underlying social construction of rape in India. He notes that the importation of "loan" words by the media in constructing rape marginalizes other sufferers other than the middle class or upwardly mobile women. Using loan words, media outlets depict class categories of victims by underscoring gender norms as well as the barriers facing raped women in India. In other words, rape construction is affected by the social-cultural contexts and ideological prisms of reporters. Furthermore, Nagar [14] questions the deification of rape victims in India which suppresses their victimhood and suffering. It follows that victims of rape or sexual violence in India are mythically characterized. We argue therefore that the portrayals of rape stories by the media more often than not, reflect power relationship in headline casting and story narration.

Scholars note that media depiction of rape victims devotes much attention to the victims and overlooks the rapist thereby presenting the victim as lacking agency and powerless. In reporting violence against women, the media are also accused of justifying the myths of sexual violence. This becomes clearer when one activates Butler's [15] notion of gender performativity and the consequences of failing to execute assigned gender roles of being submissive. Thus, it is believed that women cause rape by failing to submit their bodies. Further to this, Bonnes [3] notes that the way a South African newspaper (Grocott's Mail) reports rape perpetuates gender and racial stereotyping. While not all rape catches media attention, those in the media sustains rape myths. In the United States, Sacks et.al [16] found that local media reporting "on sexual assault might directly reinforce some of the commonly known rape myths." Meanwhile, rape myths can also discourage reportage. The perpetuation of rape myths in the media coverage of rape can engender negative 
attitude towards the victims or complete violence against women. This may further the suffering of victims of rape by refusing to report when violated.

Writing on the consequences of rape for return migration, Patil and Purkayastha [17] found that due to the permissive rape culture of girls and women in India, Indians in Diaspora, particularly those with girl-child are discouraged to return to their homeland. Indians in Diaspora view the rape culture as an expression of Indian patriarchy. This may be true depending on the perspective of the reporters of the story and whether she/he is from within the social cultural context or from without. Expatiating on this point, Bradley et al. [18] aver that writers within cultural context of rape are more likely to understand rape than the use of colonial lens in reporting rape by the western media. In deploying such colonial lens, western media are accused of privileging urban and middle class victims while marginalizing rural, poor and male victims in their reportage. They argue that the colonial view of rape constructed from western media narrowly captured the victims and perpetrators of rape in India.

To sum this review, we argue that the treatment of rape victims depend to a larger extent on the ideological leanings of different societies, the shaming culture, the spirit and form of the criminal law and the law enforcement agencies and how the media signposts a rape story.

On the nature, patterns and dynamics of rape and sexual assault, a research that was carried out by the National Crime Victim Survey (NCVS) on the Rape in America Study and The Extent, Nature, and Consequences of Rape Victimization discovered that: non-stranger or acquaintance rape was more common than stranger rape. Statistics from their findings showed that $78 \%$ of rapes/sexual assaults were perpetrated by someone known to the victims. Further investigation of perpetrator/victim relationship from their study showed that $9 \%$ of victims were raped by husbands or ex-husbands, $11 \%$ by fathers or step fathers, $10 \%$ by boyfriends or ex-boyfriends, $16 \%$ by other relatives and $29 \%$ by other non-relatives such as friends and neighbors [19]. Their findings also showed that few rapes/sexual assaults involve the use of weapon. Only about $4 \%$ of rapes/sexual assault involved the presence of fire arm and only $2 \%$ involved the use of knife. The finding suggested that rapists are more likely to gain control of their victims through deception, manipulation and betrayal of their victim's trust [19]. The report also further revealed that it was even rare for rape victims to sustain any visible physical injuries in addition to the rape and the report also stated that majority of rape go unreported as only $16 \%$ of rape was reported to the police [19]. In recent times, these figures are increasing at a geometrical rate. On perpetrators of rape, it was discovered that males constituted $99 \%$ of the offenders [19].

\section{Methods}

Method adopted was exploratory and sampling was done with the help of Nigeria Tribune Media Library in Ibadan. Punch and Sun newspapers published from January 2012 to December 2016 were analyzed. The sample size calculation was done using 1850 editions of newspapers from both the Punch and Sun [20-23]. Using articles, headlines and rape stories, rape victims and offenders; patterns of rape among others were studied and reported. Six different months each were picked for both the Punch and Sun newspapers to represent a year and subsequently for the 5 years studied. The sample size for this population was determined by multiplying the number of days/editions of newspapers by five to cover for the 5 years studied; therefore 1850 editions of newspapers from both the Punch and Sun were purposively sampled out of which 331 rape cases were 
reported and analyzed for the study. Using articles, headlines and rape stories, we analyzed characterization of rape victims and offenders; patterns of rape among others. The content analysis was employed in data presentation around the related research objectives.

\section{Findings}

\subsection{Characterizing rape victims and offenders}

This involved understanding the sex of the victim and offender, the age of both the victim and offender, the manner in which the rape was perpetuated, relationship between the victim and the offender, status of the victim and examining the concluded cases (Table 1).

Males constituted about $4 \%$ of the total number victims of rape reported for the past 5 years. On the other hand, the total number of female victims reported for the past 5 years by the two selected newspapers was three hundred and nineteen (319). In other words, females constituted about $96 \%$ of the total victims of reported rape cases within the period covered. Gill [24] has conceived rape as a criminal act that is targeted at women in order to humiliate, debase, overwhelm and control them. But Javaid [25] also found that in England and Wales, male rape are usually underreported and are affected by the masculine nature of the police subculture.

From Table 2, the total number of males was three hundred and twenty nine (329). That is males constituted about $99 \%$ of those who engaged in the act of rape for the past 5 years. On the other hand, the total numbers of females who engaged in the act of rape are two (2). This means that females constituted only about $0.6 \%$ of perpetrators of the act of rape. These findings indicate that males were more likely to engage in rape than their female counterparts. This is in line with the finding in the United States that female victims accounted for a total of $94 \%$ of all completed rapes, $91 \%$ of all attempted rapes and $89 \%$ of all attempted and completed rapes [26] (Table 3).

Victims within the age of 1-10 recorded a total of one hundred and nine (109), which is about $33 \%$ of the total victims of reported rape cases for the past 5 years. This figure is frightening when the ages of these victims are considered especially knowing that they are children. This thus means that children between ages 1 and 10 are at a high risk of falling victims to rape. Rape of this nature are scary because these children are innocent, tender, vulnerable and they are most times raped by people they trust and look up to such as teachers, uncles, fathers, step-fathers, grandfathers, neighbors, in-laws, family friends, etc. The psychological trauma they go through after this incident is more than that of adults. These victims are also at risk of contracting several sexually transmitted diseases such as STD, HIV/ AIDS and other complications such as lacerations which put their lives at risk. Daily Sun [27], reported an incident where two brothers who were teenagers raped their step-sister who was 6 years old to death.

The suspects a 19 year old Felix and his 16 year old brother Stanley were said to have had carnal knowledge of the minor by forcefully penetrating her which led to severe injuries in the victim's private part. Aside from the injuries sustained after the alleged sexual abuse, little Miss Precious later developed protruding stomach, leading to medical complications that eventually cut short the life of the victim. Also according to report, the younger brother lured his elder brother into the act. He assured his brother that there was nothing to be afraid of and that he (Stanley) had been doing it with other children in the area. 


\begin{tabular}{lcc}
\hline Sex & Frequency & Percentage \\
\hline Male & 12 & 3.6 \\
\hline Female & 319 & 96.4 \\
\hline Total & 331 & 100.0 \\
\hline
\end{tabular}

Table 1.

Sex of the victim.

\begin{tabular}{lcc}
\hline Sex & Frequency & Percentage \\
\hline Male & 329 & 99.4 \\
\hline Female & 2 & 0.6 \\
\hline Total & 331 & 100.0 \\
\hline
\end{tabular}

Table 2.

Sex of the offender.

\begin{tabular}{lcc}
\hline Age & Frequency & Percentage \\
\hline Less than a year & 1 & 0.3 \\
\hline $1-10$ & 109 & 32.7 \\
\hline $11-20$ & 153 & 46.1 \\
\hline $21-30$ & 28 & 8.4 \\
\hline $31-40$ & 12 & 3.6 \\
\hline $41-50$ & 3 & 0.9 \\
\hline $51-60$ & 1 & 0.3 \\
\hline $61-70$ & 3 & 0.9 \\
\hline 71 and above & 2 & 0.6 \\
\hline Unknown & 19 & 5.7 \\
\hline Total & 331 & 100.0 \\
\hline${ }^{*}$ By “unknown" we mean those categories of rape victims reported with unspecified age. We included them to account \\
for their victimhood in the study. However, non-inclusion of age does not make them less a victim.
\end{tabular}

Table 3.

Age of the victims.

The breakdown of trust among family members who are supposed to provide protective custodies to their eventual victims call to question the weakness or lack of moral teaching/socialization in the perpetrators. This is because teenagers and adolescents continue to be arrested for contributing to the victimization of their "sisters." All that they use is exploiting the vulnerability of the victim and in cases where the victim raises alarm, they silence them with death. This implies they understood the implication of being caught in the act or engaging in such a taboo in Nigeria. It is incest for relatives in most cultures in Nigeria to have sexual relationships. When such happens the rapist may become terribly violent and kill the victim. In a case a boy murdered his 10 year old cousin who raised the alarm for defiling her. The suspect narrated that:

Tawa met me at the passage of my house when she came to visit me on that fateful day. I asked her where she was going and she responded that somebody sent her 
somewhere but decided to check on me. Tawa latter dragged me into my room where she removed my trousers and both of us had sex on my bed. Then, she put my manhood into her private part and started shouting. Following this, I started asking her the reason for the alarm she was raising. The alarm raised by Tawa was after two minutes of sexual intercourse between us. When she started shouting, I got hold of her neck and nose and pressed them until she finally died [27].

Furthermore, victims between ages 11 and 20 recorded the highest figure with a total of one hundred and fifty three (153), which is about $46 \%$ of the total victims of reported rape for the past 5 years by the selected two newspapers. The majority of victims at this range are teenagers, a period that is marked with growth and development of sexual features. This indicates that teenagers are more prone to being victims of rape than any other age category in Nigeria. The perpetrators here are also people in key positions of trust such as fathers, step-fathers; uncles, friends, family friends, cousins, grandfathers, and the clergy. For instance, a 12-year old was impregnated by a cleric in Lagos State whom she had thought was assisting her by providing her a shelter but who later exploited her weakness/vulnerability to get what he wanted. He lured and threatened to ensure the secret was not opened up. He ensured her compliance by providing her with what she needed but soon as she became pregnant, stopped caring for her. The victim stated that:

He (Alfa) lives inside our compound. One day, he just said that I should come to his shop and I went there. He told me that I did not have a shelter; I should be sleeping inside his shop pending when I would be able to secure a place. Whenever I was sleeping in the night on the pavement, he would come around 2 am and wake me up to follow him to his shop and have sex with him. Many people in this place are aware of it. The first time he forced his private part into me, he said I should not scream. He told me I should not tell anybody but people have been seeing us. When people tried to advise me, he said I should not listen to them. He started having sex with me since I was 10 years old. He told me if I start having sex with him, he would give me money and he lured me into the corner and started touching my breasts. I wasn't happy about it and I told him to use condom, but he refused and he warned me not to tell anybody. He warned that if I tell anybody, he would use juju (charm) on me because they said that he is from Ijebu. He was giving me money but he stopped since I got pregnant.

Going further, victims between ages 21 and 30 constituted a total of twenty eight (28) that is about $8 \%$ of the total victims of reported rape cases. Also victims between ages 31 and 40 recorded a total of twelve (12), which is about $4 \%$ of the total victims of reported rape cases in the past 5 years. In their own category, victims within the age of $41-50$ recorded a total of three (3), which is about $0.9 \%$ of the total victims of reported rape cases. Also, victims between the ages of 51-60 recorded one (1) case, representing $0.3 \%$ while those aged $61-70$ and 71 and above recorded only a total of five (5) rape cases representing $2 \%$ of the entire victims. From the foregoing, the findings revealed that the younger females are more susceptible to rape than the older females. They are at their prime and more attractive to rapist than older women. They constitute a cohort that can be easily manipulated, lured, coerced and yield to threat. This is because they are more vulnerable and lack of capable guardianship. While the ages of victims are younger, what age differentials exist between the victims of rape and their rapists?

Offender demographics such as age are vital in providing information about which age grade are more likely than others to rape and where social control mechanisms can be channeled. Table 4 shows that put together the youths and middle 


\begin{tabular}{lcc}
\hline Age & Frequency & Percentage \\
\hline $13-17$ (teenagers) & 6 & 1.8 \\
\hline $18-35$ (youths) & 151 & 45.3 \\
\hline $36-55$ (middle age) & 100 & 30.2 \\
\hline 55 and above (old age) & 40 & 12 \\
\hline Unknown & 34 & 10.2 \\
\hline Total & 331 & 100.0 \\
\hline${ }^{*}$ Those in this category were not disclosed in the papers reviewed in our study. In other words, the age category was \\
unspecified in the report which shows lapses in demographic reportage of age of rapists by Nigerian newspapers. \\
\hline
\end{tabular}

Table 4.

Age of the offender.

aged (18-55) perpetrated highest percentage of rape within the period under coverage. With a percentage of $75.5 \%$ of the entire rape cases reported, these age categories constitute the deadliest in terms of rape. Reasons for its high prevalence among youths according to reports are unemployment, drug use, alcohol, coercive and sexual fantasies (resulting from pornography), peer pressure, hostility towards women (especially when the male advances are rejected), inadequate institutional support from the police and judicial system, vengeance, relationship conflict, indiscipline, wickedness and idle mind.

Refusal to date may make an offender rape a lady. This was the case when a 35 year old lured his victim out of the location where she could find help to where he is more comfortable. At the destination, he used weapon to threaten his victim and later became successful. After his arrest, he narrated:
"Actually I have interest in her and I have been eyeing her for a long time but she refused to accept my proposal. It was since two years ago immediately we moved into that house and I saw her. I then told her that she should accompany me to Ikere-Ekiti that I want to pack some of my belongings there. So, we entered the same vehicle and when we got to Ikere, I told her that I wanted to reach my site, an uncompleted building. There was nothing much that happened there. I only held her hand and said she should let us have fun together. So when I was dragging it with her, mistakenly the bottle in my hand jumped up and wounded her in her head. She then fell down; I then had sex with her. And when we got home, she reported the matter to her sister" [27].

Furthermore it is important to account for the increasing occurrence of rape among those aged 36-55. We found that midlife crisis which may make middle age men seek intimate relationship with a younger woman, boredom, work related challenges/stress (unemployment or sack), woman crisis (inability to attract the right woman) accounted for the reported rape cases among this cohort. For example inability to attract a woman owing to problem of finance made a 55 year old man impregnate a 13 year old girl. Again, the strategy is to explore the weakness of the victim in raping them. The rapist explains that:

They (the girls) sell petty consumable things and each time they come to my house, I will lure them into the house with the excuse that I want them to watch TV. Since most of them do not have one in their houses, they would be glad to come in. I would play one of my videos where people are having sex (pornographic films) and in the process convince them to have sex with me. I am so ashamed of myself 
because I have deflowered a lot of them through that process and they keep coming back for more.

Talking further on why he chose under-age girls, the rapist stated that;

the much I have given those girls is $N 200$ at a time. I felt that it is expensive spending much money on Adults. I know that it is a crime and I ask God to forgive me and give me a second chance. I was greedy that is why my wife abandoned me to my fate.

The offenders who are classified as old age ranged from 56 and above and they recorded a total of forty (40) which is about $12 \%$ of the total reported rape cases. They are mostly grandfathers, fathers, step-fathers, guardians, and community leaders. They are people that the victims trust and hold in high esteem as emblem of wisdom. We found that they rape owing to boredom, indiscipline, idle mind, wickedness and the need to have intimate relationship with younger girls. A 7-year old rape victim narrated how she was rape by a 65 -year old man.

\begin{abstract}
On Friday, my mummy was not around and he came to fetch water (like he normally does). When he was passing in front of our house, he asked me to follow him to come and collect biscuit. When I got to Baba's house, he put off my shoes and carried me to his bed. He removed my pants and lay on my chest. When I was shouting, he said I should not shout that he would give me biscuit and N20. When he finished, I saw blood coming out and Baba used a cloth to clean it. He said I should not tell anybody. He gave me biscuit and N20. He called me again the next day and did it again $[4,6]$.
\end{abstract}

This shows that the rape actualized using luring but more importantly, we argue that the occurrence of rape is the convergence of a suitable target, willing offender in the absence of capable guardianship.

\title{
4. Conclusion
}

The study has examined the coverage of rape episodes in Nigeria. Specifically, we purposively selected two national newspapers with dedicated crime reporting pages for our study. Our goal was to sift from rape coverage by the selected print media, characterization of victims and rapists. In doing this we analyzed data around the age of the victims, sex of the victims and the age of the offender and supported this by victims' and rapists' narratives.

Findings indicated that females were reported more as victims of rape than males out of the 331 rape reports covered in the dailies. Although males were also victims of rape, the percentage was about $4 \%$. This however should not be taken to mean that males are not victims of rape. What is most important is the socio-cultural environment within which rape occurs, ideological leanings of the reporters and the behavior of the criminal law in Nigeria with respect to male. We noted earlier that the law blinds it eyes against the tendency that a male can be raped in Nigeria which calls to question how male victims of rape will seek justice where their victimhood is not recognized. Furthermore, males were more prone to being perpetrators of rape than their female counterparts; however, females can also become perpetrators too. Concerning the age of victims, we found that the young adults and the middle aged were more susceptible to rape than any other age category. Conversely, the same cohorts of victims also featured prominently as highest perpetrators of rape within the 5-year period. 
Characterizing victims and offenders of rape enables us the privilege of knowing that the perpetrators of rape were mainly "familiar-foes." Few were strangers. Family members, relatives and neighbors dominated rapists who exploited the knowledge of victims' vulnerability as well as familiarity to execute rape. Apparent from this study is an implied collapse of moral community and the limits of trust in social relationships between the neighbors, parents, families, associates and friends.

Arising from the findings, rape as recorded occurred owing to mastery of the routine activities of victims, their vulnerability and the absence of capable guardianship. According to the routine activity theories, crime occurrence is the convergence of three factors: the attractive target; a willing offender; and lack of capable guardianship. The routine activities of parents which makes the girl-child find "unholy solace" in predatory "guardians" must be broken. Informal social control, which starts from the family, must incorporate security training. Victims of rape covered are mostly from the lower social economic rung of the society and this speaks to issues of power relations between the victims and the offenders not only in terms of age differentials but the limit of resistance in the most vulnerable condition. Third-party guardianship may be predatory and parents of children in the susceptible category as discovered in this study must create and secure safe environment to reduce victimization of the girl-child. At level of governance, postrape therapy is needed for victims to aid recovery of their self-worth.

\section{Author details}

Oludayo Tade* and Collins Udechukwu

Department of Sociology, University of Ibadan, Ibadan, Nigeria

*Address all correspondence to: dotad2003@yahoo.com

IntechOpen

(C) 2020 The Author(s). Licensee IntechOpen. This chapter is distributed under the terms of the Creative Commons Attribution License (http://creativecommons.org/licenses/ by/3.0), which permits unrestricted use, distribution, and reproduction in any medium, provided the original work is properly cited. (cc) BY 


\section{References}

[1] Tade O, Udechukwu C. ' 65 year old man rapes, impregnates own teenage daughter': Rape representation in two Nigeria newspapers. The Nigerian Journal of Sociology and Anthropology. 2018;16(2):33-48

[2] Rape. 2012. Available from: http:// www.soc.ucsb.edu/sexinfo/article/rape [Accessed: 11 January 2017]

[3] Bonnes S. Gender and racial stereotyping in rape coverage. An analysis of rape coverage in a South African newspaper. Grocott's Mail. Feminist Media Studies. 2013;13(2):208-227

[4] Hanafi A. 55 year old guard abducts, rapes 14 year old for four days. The Punch; 1 September 2016. p. 5

[5] Uwujare N. Pastor defiles neighbours 12 year old daughter. The Daily Sun; June 22, 2014. p. 13

[6] Ben-Nwankwo N. Tricycle rider gave me N200 after raping me. The Punch; 6 February 2016. p. 28

[7] Godwin I, Ifyede H, Sizah F. Teacher arrested for defiling 3 year old. The Daily Sun; 1 December 2016. p. 5

[8] Lees S. Carnal Knowledge: Rape on Trial. London: The Women's Press; 2002

[9] Jones H. Rape, consent and communication: Re-setting the boundaries. Contemporary Issues in Law. 2003;6 (1):23-36

[10] Oduah FM. Media coverage of rape cases in Nigeria [unpublished MSc thesis]. 2014

[11] Musbau R. Between justice system and rape epidemic. The Guardian Newspapers. 2013:53. Sunday, November 3
[12] South African Police Service. Crime situation in South Africa. 1 April 201631 March 2017. 2017. Available from: https://africacheck.org/factsheets/ guide-rape-statistics-in-south-africa/ [Accessed: 10 December 2017]

[13] National Police Service. Crime Situation Report. 2015. Available from: www.nationalpolice.go.ke/crimestatistics.html?download=26: crimereport [Accessed: 10 December 2017]

[14] Nagar I. Reporting rape: Language, neoliberalism, and the media. Discourse \& Communication. 2016:1-17. DOI: $10.1177 / 1750481315623900$

[15] Buttler J. Gender Trouble. New York: Routledge; 1990

[16] Sacks M, Ackerman Alissa R, Shlosberg A. Rape myths in the media: A content analysis of local newspaper reporting in the United States. Deviant Behaviour. 2017. DOI: 10.1080/01639625.2017.1410608

[17] Patil V, Purkayastha B. The transnational assemblage of Indian rape culture. Ethnic and Racial Studies. 2017:1-19. DOI: 10.1080/01419870.2017.1322707

[18] Bradley T, Sahariah S, Siddiqui O. A critical reading of western newspaper narratives of rape in India and their implications for feminist activism. Continuum: Journal of Media \& Cultural Studies. 2017;31(1):126-137

[19] National Crime Victimization Survey. Department of Justice, Crime Victimization. 2002. Retrieved from: http://www.ojp.usdoj.gov/bjs/cvict.htm [23 April 2006]

[20] The Daily Sun. Police officer dismissed for raping two year old girl. 6 November 2011 
[21] The Daily Sun. A virgin school girl raped by a gang of three. 31 October 2013

[22] The Daily Sun. Seventeen year old girl abducted and raped while returning from school by a gang of three. January 2014

[23] Akinkuotu E. Driver rapes 15-year old housemaid, blames the devil. The Punch; 10 April 2013

[24] Gill R. Gender and the Media. Cambridge: Polity Press; 2007

[25] Javaid A. Police responses to, and attitudes towards, male rape: Issues and concerns. International Journal of Police Science and Management. 2015;17(2):81-90

[26] Federal Bureau of Investigation. Uniform Crime Reports. 2004. Retrieved from: http://www.fbi.gov/ ucr/cius_04/ [10 April 2006]

[27] The Daily Sun. Three men rape a seven year old girl. 19 December 2013 


\title{
Experiences of Sexual and Reproductive Healthcare Professionals Working with Migrant Women Living with Female Genital Cutting in Western Australia
}

\author{
Darlene Ndasi and Kwadwo Adusei-Asante
}

\begin{abstract}
Female genital mutilation or cutting (FGM/C) is a global public health problem. The practice is particularly prevalent amongst people of African, Middle East and South East Asian descent. FGM/C creates a permanent change to the body of women. When such women migrate to other countries, they bring the associated social and health problems of FGM/C with them. As a multicultural society, Australia has many residents who come from settings in which FGM/C is prevalent. This qualitative study investigated whether healthcare professionals in Western Australia are prepared and able to provide adequate healthcare to women living with FGM/C. We found that there is a paucity of literature in Australia generally, and Western Australia more specifically, about FGM/C and the associated experiences of healthcare providers. Healthcare professionals were found to experience challenges when working with women living with FGM/C, mainly because of poor cultural sensitivity and poor levels of communication, and lacked appropriate education and training for working with women living with FGM/C. This study identified a need for empirical studies on how women living with FGM/C experience sexual and reproductive health services in Western Australia.
\end{abstract}

Keywords: female genital cutting, FGM/C, gender, phenomenology, women

\section{Introduction}

Female genital mutilation/cutting (FGM/C) is practised beyond the borders of countries where it is traditionally reported. Through the influx of asylum seekers and refugees to host countries such as Australia, FGM/C is increasingly creating challenges for healthcare professionals, who may have no or few culturally specific skills to work with its presentation in migrant women [1]. The sexual and reproductive needs of migrant women living with FGM/C are unique, and without culturally specific healthcare frameworks, meeting the healthcare needs of these women may be inhibited by cultural, environmental, and language barriers [2]. 
The World Health Organisation (WHO) classifies FGM/C into four categories: Type I, Type II, Type III and Type IV. Type III is described as the most severe, whilst Type IV is described as only symbolic and not a ritual [3]. There are two forms of Type I. Type Ia involves the intentional removal of the hood of the clitoris and seldomly occurs on its own $[4,5]$. Type Ib results in clitoridectomy-a procedure requiring the removal of the clitoral hood. Type Ib is more common and may also include the partial or complete removal of the clitoris together with that of the prepuce [6]. In Type II (excision), the clitoris and labia minora are partially removed with sharp objects, although some cultures partially cut out the labia majora and may apply ashes or herbs to stop the ensuing bleeding $[5,7]$. FGM/C Type III (infibulation) involves the removal of all external genitalia, after which the wound is fused with cat gut, thorns or surgical threads (see $[8,9])$. The clitoris may be pricked, pierced and scrapped to let blood in FGM/C Type IV. The practice represents the ritual of FGM/C in communities where FGM/C is outlawed ([10, 11]; for other forms of the practice, see $[12,13])$.

An estimated 200 million women and girls globally have undergone the FGM/C procedure, with populations with the highest percentages residing in Africa, including 1\% in Cameroon, 4\% in Ghana and Togo, and above 91\% in Egypt and 98\% in Somalia UNICEF [5]. In Europe, it is estimated that half a million women and girls are living with FGM/C [14], mostly due to the mobility of women and girls from countries that practice FGM/C $[15,16]$.

The prevalence of FGM/C in Australia is difficult to determine, although some speculate that it has been around since 1994 [17]. Whilst there appears to be a lack of research and literature around FGM/C in Australia, the increase of migrants into the country from nations where FGM/C is practised may be an indication that FGM/C prevalence is increasing in Australia [18]. It is believed that women who have experienced FGM/C arrive in Australia after the procedure has been done [19]. Tellingly, statistical analysis shows that Australia received 38,299 migrants from 11 African countries where FGM/C is highly prevalent such as Sudan $(24,082)$, Egypt (6258), Somalia (2736) and Ethiopia (5223), with the remainder from other African nations, as reported by Bourke [20, 21]. In 2010, the Melbourne Royal Hospital reported that it had seen 600-700 women living with FGM/C [20, 21]. Mathews [18] argues that the challenge of accurately establishing the prevalence and occurrence of FGM/C in Australia also stems from secrecy of the practice when compared to how it is symbolically portrayed in public ceremonies in countries that observe FGM/C (see also [16]).

A 2010 US survey revealed that sexual and reproductive healthcare professionals (SRHPs) may have knowledge about the presentation of FGM/C amongst migrants, but lacked culturally competent skills and adequate information to provide competent healthcare for women living with FGM/C [22]. Hess et al. [22] also found that negative attitudes and cultural insensitivity in SRHPs reinforce stigmatisation and isolation, resulting in poor sexual health amongst migrant women. Further, Berggren et al. [23] conducted a study in Sweden revealing that midwives attending to women living with FGM/C did not have adequate knowledge, which was evident during labour of women with infibulation. They also revealed that cultural insensitivity by healthcare professionals, poor cross-cultural communication and poor management of the labour process resulted in caesarean sections in women with FGM/C more often than was necessary.

Zaidi et al. [24] found that there is a need for healthcare professionals working with women living with FGM/C to be aware of their own cultural prejudices that may affect the wellbeing of their patients. Other researchers investigating healthcare professionals' knowledge and management of FGM/C revealed that cross-cultural training of healthcare professionals working with migrant women is imperative to 
address the knowledge gaps that hinder efficient service delivery to women living with FGM/C [25, 26]. Widmark et al. [27] suggested that the systematic involvement of infibulated women in pregnancy and birth planning frameworks could ultimately establish efficient service delivery and address knowledge gaps.

Cultural competence amongst healthcare professionals working with women living with FGM/C can also decrease stigmatisation towards these women, ultimately leading to better health outcomes for them and their families [22]. Further, Zaidi [24] cited communication between the healthcare professionals and women living with FGM/C as a major drawback to the achievement of better health outcomes for women living with FGM/C in host countries. This study aimed to investigate the experiences of healthcare professionals providing sexual and reproductive healthcare to women living with FGM/C in Western Australia. A secondary aim of this study was to contribute to the wider body of knowledge regarding healthcare professionals working with women living with FGM/C in Western Australia.

\section{Literature review}

The WHO defines FGM/C to include 'all procedures that involve partial or total removal of the external female genitalia or other injury to the female genital organs for non-medical reasons' ([3], p. 3). FGM/C is performed on children between 0 and 15 years [28]. Traditionally, it is performed at 8 years of age on average but can occur at any age. Kolawole [29] argued that FGM/C is infused with cultural, legal and medical implications, making it difficult to adequately define. Debates regarding terminology and definitions continue across all societies, social groups and cultures. Almroth et al. [30, 31] pointed to concerns amongst scholars regarding the use of terminology and the ritual of FGM/C; in particular, some scholars consider 'mutilation' (p. 457) a medically acceptable term for the practice, as it involves the removal of healthy tissue and organs without medical indication, whilst others have argued that this term denotes negative connotations and attitudes towards the procedure, and may be offensive to some cultural groups [31, 32].

We have argued elsewhere [33] that the term 'female circumcision' implies an analogy with male circumcision, which is misleading from an anatomical aspect as male circumcision only involves the removal of the glans, which by no means corresponds to the cutting of female genitalia (see also [31, 34]) - FGM/C could only be compared with male circumcision if the penis were completely amputated and the surrounding tissue removed, as the cutting experienced by girls is severe, with irreversible effects that inhibit both sexual and reproductive capacities [29].

According to Bibbings [35], 'female genital mutilation' (p. 139-149) suggests torture and violent bodily injury forced on children and unwilling women by men and women from their cultural groups for the benefit of men and their communities or groups. This study shares this viewpoint, and both the term female genital mutilation and cutting (FGM/C) have been used concurrently to take a strong stand against the procedure, whilst acknowledging the theoretical debates on the definition of FGM/C as well as cultural, social and health implications of the procedure (see [36]).

Several scholars have argued that FGM/C is a strategy that exploits female sexuality for the sexual pleasures of men [37-39]. Some authors have postulated different reasons for the occurrence of FGM/C, including marriage [39], religion [26], cultural reasons [40], ethnicity [41], maternalism [23], patriarchy, and social pressures [37]. Additionally, community attitudes towards the continuance of the practice are reported to slow and frustrate measures designed to address and potentially end the practice $[11,42]$. 
According to Almroth et al. [30, 31], there seems to be no known clinical studies that have documented the consequences of FGM/C, and the body of available literature only documents results of survey-based studies [30]. Anecdotal evidence and accounts of the consequences of FGM/C report a correlation between the procedure and adverse sexual and reproductive health outcomes [43]. Immediate and longterm sexual and reproductive health conditions have been reported in women living with FGM/C, although the severity of these conditions is believed to be dependent on the extent or the type of the procedure performed.

Diouf and Nour [44] argued that the adverse effects of Type I, Type II and Type III FGM/C can be short and long term, and possibly increase the risk factors for an HIV transmission due to the use of non-sterile instruments during the procedure. Wakabi [45] documented how surgical equipment is traditionally used numerous times on more than one initiate during the FGM/C procedure without being sterilised, increasing the risk of HIV and other blood-borne viruses (BBVs) being transmitted. Additionally, [46] argued that excessive bleeding is a complication of the FGM/C procedure, which can increase the need for medical attention (i.e., a blood transfusion); this may again increase the risk of potential HIV transmission. Although untraditional, it is based on adverse medical complications of FGM/C that the procedure came to into existence by emulating western medical practices of male genital cutting/ circumcision (MGC/M).

The medicalisation of FGM/C refers to any form of FGM/C being performed by healthcare providers, whether in private or public facilities, in the home or any other place where it may occur, in contrast to the traditional circumcision ceremony, which is usually ritualised and surrounded by public celebrations of the procedure [47]. In Kenya, for example, the medicalisation of FGM/C has become common amongst holidaymakers living in Western countries who originate from this country. Therefore, it is common amongst the Bagusii and the Kuria in the west of the country, where FGM/C is highly prevalent, and Njue and Askew $[48,49]$ reported that it has become common amongst nurses, midwives and even medical doctors to perform the procedure after consultations with a child's parents.

However, we opine that the medicalisation of FGM/C raises moral questions pertaining to whether it is a measure to protect the sexual and reproductive health of girls and women or an imperialistic promotion of a dangerous practice [50, 51]. Furthermore, the minimisation of complications during and after the procedure raises questions as to whether the medicalisation of FGM/C reduces harm or perpetuates an extremely dangerous practice [38].

A decade ago, the WHO delivered a joint statement against the medicalisation of FGM/C on the basis that it excuses and perpetuates a harmful practice, and further stated that the medicalisation of FGM/C is a dangerous and criminal practice, requiring strategies to stop healthcare providers from carrying out the procedure [52].

\section{Methods}

This project was designed as a qualitative study. According to Morse [53] and Sandellowski [54], qualitative research is particularly useful when collecting information about unknown topics, as it has the potential to provide rich and detailed descriptions of a phenomenon. The descriptive phenomenological approach in qualitative research was used as it can provide deep insights, as discussed by Liamputong $[55,56]$. Phenomenology is an approach to qualitative 
research that seeks descriptions of phenomena and how they are experienced by the actors and is effective in bringing to the fore the perceptions and experiences of individuals from their own perspectives [57]. Phenomenology allows researchers to unearth lived experiences of individuals through narratives, whilst the research takes a neutral position with no preconceived ideas of what the outcome might be (see [58-60]). Lopez and Willis [61] also suggested that the phenomenological approach in qualitative studies can examine subjective human experiences by making enquiry efficient without bias and preconceived knowledge, attitudes and values, which must be shed by the researchers prior to conducting interviews.

Purposive sampling was applied as a time-saving technique, to avoid the process of searching for specific information in a potentially broad group of possible informants. Coyne [62] suggested that purposive sampling is the most appropriate method when detailed and abundant data are required to investigate a specific phenomenon [62]. Therefore, participants were chosen based on their experiences working with patients living with FGM/C who were seeking sexual and reproductive healthcare services. The research sample was drawn from different healthcare organisations across the Perth metropolitan area, all of whom worked in various sexual and reproductive healthcare organisations.

The researchers also applied snowball sampling; six participants who met all the inclusion criteria were interviewed. The number of participants in this study was appropriate, as qualitative research does not seek to generalise but to seek and report on phenomena [63]. They ranged in age from approximately 25-60 years, with mean age of 39 years. The participants comprised four midwives, one nurse and one doctor. All worked for the government public health departments of both government and private sectors.

Face-to-face in-depth interviews were conducted at the participants' place of employment. Interviews took 30-60 minutes and were conducted using semistructured questions to guide the process and ensure relevant topics were covered. Participants were provided with a Participant Information Sheet and signed an Informed Consent Form before the interviews commenced. Signing the Informed Consent Form provided permission for the interview to be audio recorded. One joint group interview of four midwives and two individual interviews, one with a doctor and the other with a community nurse, were conducted. The joint interview was facilitated as participants had extreme time restrictions and the only available time could accommodate a joint interview comprising team members of the maternity ward department of a public hospital.

The audio-recorded interviews were transcribed within 2 weeks of collecting the data, each line being numbered and printed to allow easy examination of the content. Data reduction was conducted after the transcribing and the data coded afterwards, allowing for the categorisation of emerging themes. The printed transcriptions were examined and constantly compared with the field notes to identify emerging themes. The initial analysis was open, allowing the researchers to develop hierarchal categories across all data sets, including the field notes, to find repeated patterns of meaning. This was followed by axial coding, which allowed the researchers to connect codes, thereby establishing the relationship between all codes [63].

Open coding was initiated at the beginning of data analysis, with the researchers examining and colour-coding field notes to identify themes and then sequentially numbering all the lines in the transcribed interviews. In coding the data, Nvivo 10 and Microsoft Word were used. This study was approved by the Curtin University Research Ethics Committee. 


\section{Findings}

\subsection{Communication difficulties}

Anis et al. [64] argued that placing patients at ease in an environment so they are able to speak about their FGM/C is a key to provide consistent care and positive outcomes. Participants expressed concerns regarding poor communication between their patients and themselves. The difficulties in communication were both verbal and non-verbal, which included the refusal by patients to respond to questions when asked if they lived with FGM/C. Participants reported that they also refused examinations when they presented at the sexual and prenatal clinics. This led to the late discovery of the presence of FGM/C in women who had presented with pregnancy and labour. The following excerpts are from the interviews conducted with the SRHPs:

They don't talk about it ... but they don't talk about it ... they know what is happening to them ... the very first one that I saw I was really shocked, because there was only an opening you could only let a finger in ... she knew she had a problem ... but she didn't talk of what had happened to her ...

Another midwife made the following statement: 'they would refuse all vaginal exams ... they don't tell anyone that it has been done to them ... and they just arrive ... here!' Another participant commented:

Well ... hopefully, that should be identified at the antenatal clinic ... but as it goes ... don't tell us ... if they refuse examination at the antenatal, which a lot of them do, we don't find out until they are in labour...

The professional health workers expressed how some of the patients were not aware of the FGM/C themselves, that it had been done when they were too young and that they may not have even known that they had it. One of them reported that she saw no need to talk about FGM/C with these patients. She also expressed her view that it was not her place to talk about FGM/C as she feared there could be implications in speaking about it with her patients. She expressed a deep lack of confidence in communication with her patients living with FGM/C:

\footnotetext{
I wonder if it is helpful for me to point out to them that they have a problem which is not in one of my problem categories ... yeah ... They would be as how they have always been ... they won't actually know any difference ... I don't know what intervention is of benefit ... Maybe that is what I need to learn more about ... what evidence is there towards intervention? ... asking those questions is important but I don't know how ... but I do feel that even if someone says that, this is ok to talk about it ... I can still get myself into a lot of trouble ... and I think I ... bring up a subject that is very sensitive and very difficult ... that I actually don't have very much skill to help with ... then I would cause harm ... and so often I won't mention whether they may or may not perceive they have a problem is sometimes more harmful than good ... and yet I recognise that I may be missing an opportunity ... to discuss ... but they may not have the courage to discuss ...
}

Participants reported that they felt inadequately equipped to communicate effectively with women living with FGM/C and suggested that healthcare professionals with the same cultural background as their patients would be appropriate to provide services to women living with FGM/C. It was also suggested that 
culturally specific healthcare to women living with FGM/C would promote efficient communication between healthcare professionals and their patients. A doctor made this statement:

I guess my thinking is that someone who is from the same culture or background would understand that background and would have more ability to ... perhaps ask the right questions ... so it is that sense of someone who understand me ... so I won't go to a man for a pap smear ... someone who understands women's health ... I think the similar would be the case ... to talk to someone who thinks this is barbaric ... to do that to my daughter... how can I go to that person for help? I need to speak to someone who understands the background and the pressure ...

Additionally, participants reported that they were concerned about their patient's capacity to comprehend the medical information and advice provided. One midwife expressed concern about the ability of the patients to clearly understand what was being communicated to them during service delivery:

I'm not $100 \%$ sure women will know what we are asking them ... you know ... have you been cut? ... they may not understand what we are asking ... and they may not have knowledge of what has been done ... a youth worker ... had FGM ... and she didn't know because she didn't know how a normal vagina would look like ... She didn't know she had been cut ... a doctor had to examine her to see if she had been cut.

Another participant strongly emphasised the importance of efficient communication between patients and healthcare professionals: 'Yes. Communication is the key.'

Communication difficulties between participants and their patients were discussed by participants as barriers to efficient sexual and reproductive health service delivery. The reasons for these communication barriers were reported to stem from cultural conflict between healthcare professionals and their patients.

\subsection{Cultural conflict}

Several of the participants reported that they viewed the procedure of FGM/C in their patients as barbaric, incomprehensible, oppressive and dangerous and expressed sadness and anger at the pain that women with FGM/C have to endure during delivery. However, despite these feelings of sympathy, they experienced frustration, shock and anger that these women do not speak about FGM/C even when they are asked. A doctor made this statement:

it makes me sad that that happens ... to people who don't have the ability to step out of their culture ... they don't go, 'Oh my God, we are cutting a baby on a sensitive area! That can't be right!' And yet, I have days I reflect on tradition and things that have been done for a long time ... then there must be some reason behind it even though to my ... outsider position I am looking at it, and it is completely wrong.

Another participant stated:

The sensitivity is of course related to sexuality; it is related to cultural identity and that whole sensitivity is multiplied by the fact that we are medical professionals from the other culture ... And I think if it was my culture, I would feel I have the 
right to ask ... in the same way a woman can ask a woman about periods and talk about sex in a way that a man doctor can't ask that question ... But I come from a different culture and I am asked questions about a practice that people from that culture, which is new to this country, they realise that the practice is forbidden and that there is overriding condemnation of it. And they may even feel that me asking a neutral question could sound like condemnation.

A midwife described with profound sadness how she witnessed a woman suffer during childbirth: 'Oh, I was upset ... when I saw the poor girl ... I told you she had an opening only one centimetre. We couldn't even find her urethra ... It is very sad.'

Another midwife was clearly psychologically disturbed by FGM/C in her patients:

It makes me mad ... it makes me very sad that the woman has had to endure it ... especially if it was done in countries with basic tools ... knives, and I think, that is a small child that it was done to and I ... the pain and why you do it?

\subsection{Re-infibulation and the medicalisation of FGM/C}

Re-infibulation occurs after childbirth and returns de-infibulated women back to the previous state of infibulation; it is argued to have no benefits (e.g., [51, 65]). Statements were made by participants that women requested re-infibulation after childbirth. One midwife described how a doctor carried out re-infibulations after birth, giving him a large clientele of patients who preferred his services, which is in contrast to the typical cultural practice of women who are living with FGM/C, who usually have a preference for female healthcare providers $[22,66]$. This midwife said:

\footnotetext{
I know ... when I worked at XXXX hospital, one of the doctors used to look after a number of Middle Eastern ladies because he spoke their language, and there was a high proportion of FGM/C in these ladies ... and at one stage, I know he was sewing them back as they were ... because they were requesting it ... before he was talked to about all the legalities...
}

In non-traditional rituals, as occurred in Western societies, clinicians who support the practice of FGM/C often perform re-infibulation, creating a situation where FGM/C is medicalised [18]. This has been highly controversial within the WHO [52], which has called for prosecutions of clinicians who perform FGM/C, in line with the rationale that there is no excuse whatsoever for the facilitation of any form of FGM/C by medical practitioners [52]. Whilst the campaign against FGM/C has been highly visible within the traditional locations where FGM/C is practised (e.g., sub-Saharan Africa), in Western countries, clinicians are still faced with poor training. A lack of access to and knowledge of clear clinical guidelines for the treatment of women living with FGM/C is evident, which may increase the likelihood of re-infibulation occurring $[67,68]$.

Participants in this study described how some women would travel for long overseas holidays to perform re-infibulation and also have their newborn daughters circumcised. The following statements were made by participants:

XXXX hospital now refer women, any women who have had daughters, to social workers to ensure they don't disappear for long to circumcise their daughters ... and they are trying to introduce it here: any woman that has a female baby is followed up ... they have training for social workers ... because they have specific training ... to ensure these women do not disappear for long periods of time so the same thing is not done on them. 
Another midwife made the following statement: 'she had previous Type III FGM ... and the social worker sent me an email saying, "Can you talk to her about it, she has got two daughters and we want to make sure she doesn't cut them?"”

Some of the statements made by the participants identify the need for culturally specific sexual and reproductive healthcare to meet the needs of women living with FGM/C.

\title{
4.4 Culturally specific healthcare
}

The participants expressed the need for women living with FGM/C to have access to culturally specific healthcare, reporting that this was lacking in the public health system in Western Australia. Participants described how healthcare professionals find it difficult to understand the culture of FGM/C and therefore feel inadequate in their capacity to provide sexual and reproductive health services in an efficient manner to women living with FGM/C. A doctor expressed how culturally specific healthcare would minimise conflict in the relationship between women living with FGM/C and healthcare professionals by training professionals with a similar cultural background as the patients to provide efficient healthcare:

\begin{abstract}
it is a specialised area and accessing specialised services is important by those people who are fairly trained to help ... even within gynaecology, you wouldn't refer them to any gynaecologist ... just those who are more experienced, those who are more able to help. Psychologists would have very little experience within that area, counselling and some would be very well experienced ... I think there needs to be more conversations about it, and probably more training by people who are very much from that culture and understand all the in's and out's. And then give good advice as to how someone who is not from that culture might approach the issue. Because within our own culture we have many ideas of healthcare, even within one culture every person is different. It isn't helpful if doctors are trained to provide the wrong approach.
\end{abstract}

It appears therefore that clinicians with experience and backgrounds where FGM/C is traditionally practised can offer invaluable support to women presenting with FGM/C at sexual and reproductive health services in Western Australia. These clinicians could be targeted for specialised training in numbers that can appropriately cater for sexual and reproductive health needs of women living with FGM/C.

\section{Complications of FGM/C in sexual and reproductive health provisions}

All participants stressed the importance of consistently screening pregnant women for FGM/C and clearly recording the information at the antenatal care stage before women go into labour. The participants stated that this could help prevent complications during childbirth, such as prolonged labour and unplanned caesarean sections and episiotomies. Routine screening and the correct use of information were reported to facilitate appropriate planning and the prevention of trauma during childbirth. However, it was reported that this was often hindered by women's refusal to be physically examined and by the poor recording of information. This is exemplified in this excerpt from one midwife:

One of the doctors is the one who usually do ... the anterior cuts ... they actually do cut where they have been stitched ... the registrar would decide where the cut's gonna 
[sic] be ... exactly where they will be placed ... someone came in labour and no one knew the woman had FGM ... and then it is bit of panic, and what can we do ... Type III - a baby is not gonna [sic] come out ... normally, if it is Type III, you would do one cut up, and then two up that way, and then afterwards sew the edges ... so that it is not bleeding ... and then do construction work afterwards.

Another midwife concurred, adding the following:

and this poor girl had to have three episiotomies ... one anterior and two posterior, to get her baby out. Because the baby was pressing against her vaginal wall I guess ... and it started opening that diameter ... a centimetre ... the baby was never going to come out, so she had three cuts! And we had to get one of our consultants to come and do the delivery, because she needed a lot of work, and repair work done ... so she had to go to theatre to get everything repaired, and hopefully, next time she'd have another baby, she should've been ok.

These experiences were also reported to affect other important medical procedures. A doctor expressed difficulties when conducting pap smear examinations on infibulated women:

so the first lady that I ever met ... I was quite convinced that ... that was what I was seeing ... she had a very severe form of uterine prolapse ... which sounds like shouldn't have happened ... cause she had been stitched all up ... and when this lady came, I could see that she had been ... eh ... I am actually sure whether it would have been Type II or Type III, but certainly stitched together for the large part. So the pap smear was difficult ... so I didn't know whether to tell her that's the reason or I didn't know whether to tell her whether it is due to menopause ... so it is not really not relevant to say why ... so say sorry it hurts and I just do what I need to do.

Cross-cultural training as evidenced in this data is a key to efficient services to women presenting to sexual and reproductive health services with FGM/C. SRHPs' experiences of caring for women with FGM/C in Western Australia reveal many frustrations and difficulties of providing care for these women. Appropriate training and policy framework and clear clinical guidelines for the care of women living with FGM/C are imperative in meeting the special needs of these women. The value of adequate experience, knowledge and skills in this area cannot be overstated.

\subsection{Training needs}

The participants reported their concerns regarding the inadequate training available for working with women living with FGM/C. All of the participants stated that the existing training was inadequate and, additionally, that they were not provided with adequate time or support from their employers to participate. They also commented on the lack of an adequate curriculum. One participant said:

more training would be useful ... I know there is some training based in XXXX hospital ... and I had an opportunity to do some of that training yesterday ... and ... I mean ... it is important to do more training ... sometimes I think training would make me understand this is Type II ...

It was also suggested by the participants that professionals who have backgrounds working with women living with FGM/C would be ideal if trained as 
peer educators for women living with FGM/C, improving the processes of service delivery. A doctor made this statement:

probably more training by people who are very much from that culture and understand all the in's and out's... And then give good advice as to how someone who is not from that culture might approach the issue. Because within our own culture, we have many ideas of healthcare, even within one culture every person is different. It isn't helpful if doctors are trained to provide the wrong approach ...

Other participants stated the need for integrated curricula in tertiary and professional training courses for doctors and midwives to enable them to acquire adequate skills for addressing FGM/C in their practice. One participant commented that the training is extremely basic and only provides limited information on FGM/C:

\begin{abstract}
I think focusing less on the types and pictures ... and focusing more on the effects that it has on the women's lives... and how we can talk to people about it, and how we can educate people ... but it is more about what can we do ... educating ourselves than rather just analysis of FGM ... But the fact that a child is screaming ... it makes me sad that they are living with it and the effects it has on people. It makes me want to know more and what can I do ... I used to examine women but in this role I am currently doing antenatal care ... which is why I need to know how to ask these questions.
\end{abstract}

Another concern was expressed regarding the content of the existing training. It was reported that, for a long time, the same content has been delivered yearly, with nothing new incorporated. A midwife stated:
she [the trainer] would come and talk to our staff, she would literally take you through the types ... the medical side, and same thing every year ... same PowerPoint presentation, every year. And you knew you were never gaining anything from it ... every year, but the same thing ... quite a number of years ... probably five years ... listening to the same thing ... being delivered.

A midwife agreed and stated that, 'It's usually the same information ... it hasn't changed.' Some of the participants also expressed the need to be provided with training opportunities and supported by their employers if they were to be well equipped to deal with FGM/C in their patients. One midwife said:

I used to be a staff development officer at XXXX hospital, and the staff are inundated with 'you have to do this, you have to do that' ... (lack time for training), and plus you have to do your work ...

There is a need for healthcare policies to integrate appropriate modules in curricula for training healthcare professionals to facilitate efficient and appropriate service delivery to women with FGM/C. This has to be based on the cultural diversity in Western Australia and the larger Australian society.

\title{
6. Conclusion
}

This study focused on the experiences of healthcare professionals providing sexual and reproductive healthcare to women living with FGM/C in Western Australia and aimed to contribute to the wider body of knowledge regarding healthcare professionals working with women living with FGM/C in Western 
Australia. Individuals reported both unique experiences and commonalities within the context of their interviews. SRHPs providing services to women living with FGM/C in Western Australia identified a gross lack of adequate training services capable of equipping SRHPs to expertly meet the needs of women living with FGM/C. Significant changes are required to provide adequate care for women living with FGM/C in Western Australia.

\section{Acknowledgements}

We wish to acknowledge Curtin University's School of Public Health where the original thesis that informed this paper was submitted. The work of Mr. Matt Tilley of Curtin University's School of Public Health who supervised the original thesis is also acknowledged.

\section{Author details}

Darlene Ndasi and Kwadwo Adusei-Asante*

School of Arts and Humanities, Edith Cowan University, Western Australia

*Address all correspondence to: k.adusei@ecu.edu.au

\section{IntechOpen}

(C) 2020 The Author(s). Licensee IntechOpen. This chapter is distributed under the terms of the Creative Commons Attribution License (http://creativecommons.org/licenses/ by/3.0), which permits unrestricted use, distribution, and reproduction in any medium, provided the original work is properly cited. (cc) BY 


\section{References}

[1] Thierfelder C, Tanner M, Bodiang CMK. Female genital mutilation in the context of migration: Experience of African women with the Swiss health care system. European Journal of Public Health. 2005;15(1):86-90

[2] Dundek LH. Establishment of a Somali doula program at a large metropolitan hospital. The Journal of Perinatal \& Neonatal Nursing. 2006;20(2):128-137

[3] WHO. Female genital mutilation: A joint WHO/UNICEF/UNFPA statement. Geneva: World Health Organization; 1997

[4] Obermeyer CM. Female genital surgeries: The known, the unknown, and the unknowable. Medical Anthropology Quarterly. 1999;13(1):79-106

[5] UNICEF. Female Genital Mutilation/ Cutting: A Statistical Exploration. New York: United Nations Children's Fund; 2005

[6] Toubia N, Izette S. Female Genital Mutilation: An Overview. Geneva: World Health Organization; 1998

[7] Obermeyer CM. The health consequences of female circumcision: Science, advocacy, and standards of evidence. Medical Anthropology Quarterly. 2003;17(3):394-412

[8] Abdulcadir J, Irion O, de Tejada BM. Female genital mutilation/cutting type IV in Cambodia: A case report. Clinical Case Reports. 2015;3(12):979-982. DOI:10.1002/ccr3.403

[9] Momoh C. Female genital mutilation. Current Opinion in Obstetrics and Gynecology. 2004;16(6):477-480

[10] Bagnol B, Mariano E. Vaginal practices: Eroticism and implications for women's health and condom use in Mozambique. Culture, Health \& Sexuality. 2008;10(6):573-585

[11] Newland L. Female circumcision: Muslim identities and zero tolerance policies in rural West Java. Women's Studies International Forum. 2006;29(4):394-404

[12] Tamale S. Eroticism, sensuality and 'women's secrets' among the Baganda. IDS Bulletin. 2006;37(5):89-97

[13] United Nations. Eliminating Female Genital Mutilation: An Interagency Statement OHCHR, UNAIDS, UNDP, UNECA, UNESCO, UNFPA, UNHCHR, UNICEF, UNIFEM, WHO. Geneva: World Health Organization; 2008

[14] Van Baelen L, Ortensi L, Leye E. Estimates of first-generation women and girls with female genital mutilation in the European Union, Norway and Switzerland. The European Journal of Contraception \& Reproductive Health Care. 2016;21(6):474-482

[15] Evans C, Tweheyo R, McGarry J, Eldridge J, Albert J, Nkoyo V, et al. Improving care for women and girls who have undergone female genital mutilation/cutting: Qualitative systematic reviews. Health Services and Delivery Research. 2019;7(31). Available from: https://www.ncbi.nlm.nih.gov/ books/NBK546416/ DOI: 10.3310/ hsdr07310

[16] Ogunsiji O. Female genital mutilation (FGM): Australian midwives' knowledge and attitudes. Health Care for Women International. 2015;36(11):1179-1193

[17] Yoder PS, Khan S. Numbers of Women Circumcised in Africa: The Production of a Total; 2008

[18] Mathews B. Female genital mutilation: Australian law, policy 
and practical challenges for doctors. Medical Journal of Australia.

2011;194(3):139-141

[19] Knight R, Hotchin A, Bayly C, Grover S. Female genital mutilationexperience of the Royal Women's hospital, Melbourne. Australian and New Zealand Journal of Obstetrics and Gynaecology. 1999;39(1):50-54

[20] Bourke, E. Female Circumcision Happening in Australia. ABC News; 6 February 2010. Available from: http://www.abc.net.au/news/ stories/2010/02/06/2812147.html

[21] Bourke E. Female circumcision happening in Australia. ABC

News 2010; 6 Feb. Available from: http://www.abc.net.au/news/ stories/2010/02/06/2812147.html

[22] Hess RF, Weinland J, Saalinger NM. Knowledge of female genital cutting and experience with women who are circumcised: A survey of nursemidwives in the United States. Journal of Midwifery \& Women's Health. 2010;55(1):46-54

\section{[23] Berggren V, Salam GA,} Bergström S, Johansson E, Edberg A-K. An explorative study of

Sudanese midwives' motives, perceptions and experiences of re-infibulation after birth. Midwifery. 2004;20(4):299-311

[24] Zaidi N, Khalil A, Roberts C, Browne M. Knowledge of female genital mutilation among healthcare professionals. Journal of Obstetrics and Gynaecology. 2007;27(2):161-164

[25] Ahlberg BM, Krantz I, Lindmark G, Warsame M. 'It's only a tradition': Making sense of eradication interventions and the persistence of female 'circumcision' within a Swedish context. Critical Social Policy. 2004;24(1):50-78. DOI: $10.1177 / 0261018304241003$
[26] Leval A, Widmark C, Tishelman C, Maina Ahlberg B. The encounters that rupture the myth: Contradictions in midwives'descriptions and explanations of circumcised women immigrants'sexuality. Health Care for Women International. 2004;25(8):743-760

[27] Widmark C, Leval A, Tishelman C, Ahlberg BM. Obstetric care at the intersection of science and culture: Swedish doctors' perspectives on obstetric care of women who have undergone female genital cutting. Journal of Obstetrics and Gynaecology: the Journal of the Institute of Obstetrics and Gynaecology. 2010;30(6):553-558

[28] Zurynski Y, Phu A, Sureshkumar P, Cherian S, Deverell M, Elliott EJ. Female genital mutilation in children presenting to Australian paediatricians. Archives of Disease in Childhood. For Australian Paediatric Surveillance Unit Female Genital Mutilation Study Steering Committee. 2017;102(6):509-515. DOI: 10.1136/archdischild-2016-311540

[29] Kolawole OM. Female Genital Mutilation: When Culture and Human Rights Collide: An Analysis of Asylum and Refugee Law. London: Webster University; 2011

[30] Almroth L, Bedri H, El Musharaf S, Satti A, Idris T, Hashim MSK, et al. Urogenital complications among girls with genital mutilation: A hospitalbased study in Khartoum. African Journal of Reproductive Health. 2005;9(2):118-124

[31] Almroth L, Elmusharaf S, El Hadi N, Obeid A, El Sheikh MA, Elfadil SM, et al. Primary infertility after genital mutilation in girlhood in Sudan: A case-control study. The Lancet. 2005b;366(9483):385-391

[32] Elmusharaf S, Elhadi N, Almroth L. Reliability of self reported form of 
female genital mutilation and WHO classification: Cross sectional study. BMJ. 2006;333(7559):124

[33] Ndasi MD, Adusei-Asante K. Female Genital mutilation/cutting: A conceptual discussion. Advances in Social Sciences Research Journal. 2019;6(11):232-246

[34] Earp BD. Female genital mutilation and male circumcision: Toward an autonomy-based ethical framework. Medicolegal and Bioethics. 2015;5:89-104

[35] Bibbings LS. Female genital mutilation: Whose problem, whose solution?: Mutilation or modification? British Medical Journal. 2006;333(7561):259-260

[36] Earp BD, Johnsdotter S. Current critiques of the WHO policy on female genital mutilation. International Journal of Impotence Research. 2020:1-14. DOI: 10.1038/s41443-020-0302-0

[37] Abusharaf RM. Introduction:

The custom in question. In: Abusharaf RM, editor. Female Circumcision: Multicultural Perspectives. Philadelphia, PA: University of Pennsylvania Press; 2006. pp. 1-25

[38] Hilber AM, Kenter E, Redmond S, Merten S, Bagnol B, Low N, et al. Vaginal practices as women's agency in sub-Saharan Africa: A synthesis of meaning and motivation through meta-ethnography. Social Science \& Medicine. 2012;74(9):1311-1323

[39] Rahman A, Toubia N. Female Genital Mutilation. A Practical Guide to Worldwide Laws and Policies. London, UK: Zed Books; 2000

[40] Jones SD, Ehiri J, Anyanwu E. Female genital mutilation in developing countries: An agenda for public health response. European Journal of Obstetrics \& Gynecology and Reproductive Biology. 2004;116(2):144-151

[41] Idowu AA. Effects of female genitalmutilation on human rights of women and female children: The Nigerian situation. Journal of International Studies. 2008;8:13-26

[42] Livermore L, Monteiro R, Rymer J. Attitudes and awareness of female genital mutilation: A questionnaire-based study in a Kenyan hospital. Journal of Obstetrics and Gynaecology. 2007;27(8):816-818

[43] Balk D. To marry and bear children? The demographic consequences of infibulation in Sudan. In: Female 'Circumcision'in Africa. Culture, Controversy and Change. London: Lynne Rieneuer Publishers; 2000. pp. 55-71

[44] Diouf K, Nour N. Female genital cutting and HIV transmission: Is there an association? American Journal of Reproductive Immunology. 2013;69:45-50

[45] Wakabi W. Africa battles to make female genital mutilation history. The Lancet. 2007;369(9567):1069-1070

[46] Yount KM, Abraham BK. Female genital cutting and HIV/AIDS among Kenyan women. Studies in Family Planning. 2007;38(2):73-88. DOI: 10.1111/j.1728-4465.2007.00119.x

[47] Kimani S, Shell-Duncan B. Medicalized female genital mutilation/ cutting: Contentious practices and persistent debates. Current Sexual Health Reports. 2018;10(1):25-34

[48] Njue C, Askew I. Medicalization of Female Genital Cutting among the Abagusii in Nyanza Province. Kenya: Washington DC: Population Council Frontiers in Reproductive Health; 2004 
[49] Njue C, Askew I. Medicalization of Female Genital Cutting among the Abagusii in Nyanza Province, Kenya; 2004

[50] Pearce AJ, Bewley S. Medicalization of female genital mutilation. Harm reduction or unethical? Obstetrics, Gynaecology and Reproductive Medicine. 2014;24(1):29-30

[51] Serour G. Medicalization of female genital mutilation/cutting. African Journal of Urology. 2013;19(3):145-149

[52] WHO. Eliminating Female Genital Mutilation: An interagency statementOHCHR, UNAIDS, UNDP, UNECA: UNESCO, UNFPA, UNHCR, UNICEF, UNIFEM, WHO. Geneva: WHO; 2008

[53] Morse JM. Strategies for sampling. In: Morse JM, editor. Qualitative Nursing Research: A Contemporary Dialogue. Sage Publications, Inc; 1991. pp. 127-145. DOI: 10.4135/9781483349015.n16

[54] Sandelowski M. Focus on research methods-whatever happened to qualitative description? Research in Nursing and Health. 2000;23(4):334-340

[55] Liamputtong P, editor. Research Methods in Health: Foundations for Evidence-Based Practice. Oxford: Melbourne; 2010

[56] Liamputtong P. Research Methods in Health. Foundations for EvidenceBased Practice. South Melbourne: Oxford University Press; 2010

[57] Groenewald T. A phenomenological research design illustrated. International Journal of Qualitative Methods. 2004;3(1)

[58] Smith JA, Flowers P, Larkin M. Interpretative Phenomenological Analysis: Theory, Method and Research. London: Sage; 2009
[59] Gubrium A. "I Was My Momma Baby. I Was My Daddy Gal”: Strategic Stories of Success. Narrative Inquiry. 2006;16(2):231-253

[60] Kvale S, Brinkmann S. Interviews: Learning the Craft of Qualitative Research Interviewing. Sage; 2009

[61] Lopez KA, Willis DG. Descriptive versus interpretive phenomenology: their contributions to nursing knowledge. Qualitative Health Research. 2004;14(5):726-735. DOI: $10.1177 / 1049732304263638$

[62] Coyne IT. Sampling in qualitative research. Purposeful and theoretical sampling; merging or clear boundaries? Journal of Advanced Nursing. 1997;26(3):623-630

[63] Liamputtong P. Research Methods in Health: Foundations for Evidence-Based Practice. Oxford University Press; 2013

[64] Anis TH, Aboul Gheit S, Awad HH, Saied HS. Effects of female genital cutting on the sexual function of Egyptian women. A cross-sectional study. The Journal of Sexual Medicine. 2012;9(10):2682-2692

[65] WHO. Women and Health: Today's Evidence Tomorrow's Agenda. World Health Organization. Geneva: WHO; 2009. $91 \mathrm{p}$

[66] Garvey-Graham AM. Midwives' Experiences of Caring for Women during Childbirth Who Have Undergone Female Genital Mutilation: An Interpretative Study (Masters). Wellington: Victoria University of Wellington, 2008. Retrieved from: http://hdl.handle.net/10063/339

[67] Porterfield SP. Female genital cutting. The Journal for Nurse Practitioners. 2006;2(4):256-260

[68] Toubia N. Female circumcision as a public health issue. New England Journal of Medicine. 1994;331(11):712-716 


\title{
Don't Objectify Me!: Sexual Self-Monitoring, Coping, and Psychological Maladjustment
}

\author{
Catherine Baggett, Craig Nagoshi and Julie Nagoshi
}

\begin{abstract}
Undergraduate college students (283 females, 127 males) completed surveys aimed at measuring positive sexual awareness vs. sexual self-monitoring, coping styles, and psychopathological symptoms. Positive sexual awareness significantly positively correlated with adaptive coping styles but did not otherwise correlate with psychopathological symptoms. Sexual self-monitoring was significantly positively correlated with somatization, depression-anxiety, and avoidant coping in women but not men. Bootstrapped mediation analyses indicated that the relationships between sexual self-monitoring and somatization, depression-anxiety, and eating disorder symptoms were significantly mediated by avoidant coping in women but not in men. These results were explained in terms of Objectification Theory, suggesting that women who experience sexual objectification are more likely to engage in avoidant coping, thus increasing their risk of developing psychopathology. Findings are discussed in terms of broader issues of the disempowering effects of objectification.
\end{abstract}

Keywords: gender, sexual monitoring, coping, psychological maladjustment, objectification theory

\section{Introduction}

While men and women do not significantly differ in prevalence for most major mental disorders, such as schizophrenia and bipolar disorder, women suffer from anxiety, depressive, and post-traumatic stress disorders at higher rates than men [1-3]. One possible reason for this disparity in mental illness between men and women may be attributed to the unique experiences of women in Western society. Though women have made great strides towards equality in our society, there are still fundamental differences in the ways in which men and women are treated. Women suffer higher rates of domestic violence, sexual assault, and sexual abuse than do men [4] and are often relegated to roles as caregivers or helping professionals in both their career options and their roles at home. Furthermore, media portrayals of women (such as in advertisements and popular film) tend to oversexualize women and focus on their relationships to men rather than give them agency and character in their own right. Such objectification of women by society may create experiences for women which contribute to higher rates of anxiety, depression, and PTSD in women. 
One cannot study gender roles and the effects of society on these roles without discussing the intersecting role played by women's sexuality. Throughout history, sexuality has been used to control or define women. In her book The Purity Myth, Valenti [5] comments on the long history of judging women as virtuous based on their sexual "purity". Furthermore, sexual objectification has been used as a tool to strip women of their power by using sexuality and sexual appeal to define a woman's value and worth to others. In extreme cases, because women are treated as commodities to be consumed by men, sexual objectification has been used to justify violence against women, including sexual and intimate partner violence. However, if sexual objectification is used to subjugate women, sexual agency may be key to women's empowerment.

To be an agent is to have power over one's own body and actions. Agents are able to act on the world. To be an agent means to have the power to determine one's own identity and worth, rather than have them defined by someone else. To have sexual agency is to not only have control over one's body and sexual behavior, but also to have the confidence and awareness to use that control. Such power and control over one's sexuality has its benefits. In their commentary, Rosen and Bachmann [6] stated that the relationship between sexual satisfaction and well-being in women was an important and under-studied topic in health psychology.

Across studies, Rosen and Bachman [6] noticed a strong positive relationship between a woman's reported happiness and sexual satisfaction. This relationship has found continued support in the literature. Holmberg et al. [7] reported that sexual satisfaction was a predictor of well-being in women in both same-sex and mixed-sex relationships. Donaghue [8] found that a passionate/romantic sexual self-schema (one which indicated a greater interest and enjoyment of sex) was significantly associated with positive affect and greater life satisfaction. In one longitudinal study, sexual well-being was found to have not only a greater association with well-being in women over time, but also well-being from moment to moment [9]. Furthermore, this relationship has not only been found in America, but across countries. Carrobles et al. [10] found that, in a sample of Spanish women, greater sexual assertiveness, sexual confidence, and frequency of orgasm all predicted a greater sexual satisfaction, which in turn predicted a greater perception of wellbeing. In a survey of 13,882 women across 29 different countries, Laumann et al. [11] found a positive relationship between sexual satisfaction and psychological well-being across all countries. Interestingly, a study by Owen et al. [12] found that students who reported more depressive symptoms also reported improved wellbeing after engaging in casual sex, while students who reported fewer depressive symptoms reported decreased well-being after casual sex. This suggests that sex can be used as a tool for emotional regulation, lending further support to the relationship between sexuality and well-being. Across cultures and ages, sexual satisfaction has been found to have a strong relationship to psychological well-being.

\subsection{Sexual monitoring, objectification theory, and women's mental health}

A negative aspect of sexual consciousness, however, is self-consciousness and monitoring of one's sexual desirability. In women, such sexual self-monitoring has been found to be associated with lower sexual assertiveness and sexual activities [13]. Sexual self-monitoring is an important aspect of the self-consciousness about one's body image. Sexual self-monitoring is much more prevalent in women than men and is predictive of a range of sexual problems and sexual dissatisfaction in women [14]. Sexual monitoring strips a woman of her sexual agency by making her self-worth dependent on the judgments of others, and this disempowerment may be associated with decreased well-being in women. 
This phenomenon can be explained by Objectification Theory. Objectification Theory uses a feminist theoretical framework to understand how a woman's experience is influenced by a culture which objectifies the female body [15]. Objectification Theory posits that the experience of being treated as a "body" to be consumed by others (i.e., an object) results in a number of adverse psychological experiences for women [15]. Objectification occurs through the over-sexualization of women's bodies through obvious means, such as sexualization in the media, and also through more subtle means, such as the interactions between women and the men who engage in behaviors, such as catcalling and "elevator eyes" or the "objectifying gaze" [15]. In contrast to sexual agency, men who objectify women are more likely to report pressuring/coercing women for sex, while women who feel objectified are more likely to report being pressured/coerced for sex [16].

Because women are often treated as consumable objects, they are evaluated by their attractiveness to others, rather than their inherent dignity and worth as a person. Through self-objectification, women internalize objectifying messages from society, thus diminishing the worth of their personal attributes and focusing heavily on their physical attributes. As a result, women often feel ashamed of their bodies and engage in unhealthy behaviors, such as disordered eating, in an attempt to improve their attractiveness to others $[17,18]$. Even in girls as young as 6 years old, self-objectification has been found to affect eating habits and body anxiety [19].

Increasing evidence has shown that self-objectification negatively affects a woman's mental health $[20,21]$. In their comprehensive review of objectification theory, Tiggeman and Williams [21] found that self-objectification strongly predicted depressed mood and disordered eating in young women, as well as, though less strongly, predicting sexual dysfunction. Self-objectification has also been found to significantly predict the development of disordered eating in both adolescent girls and young women $[18,20]$. The body shame and appearance anxiety caused by self-objectification have been shown to increase depressed mood [22]. Furthermore, Carr et al. [23] argue that objectification of women's bodies can also exacerbate serious mental illnesses, such as schizophrenia and borderline personality disorder, by further marginalizing women whose mental illness has already marginalized them.

\subsection{Objectification theory, objectification, and coping}

Objectification Theory can provide insight into the link between objectification and mental illness in women. It may be that objectification interferes with a woman's ability to cope with stressors, resulting in a higher likelihood of developing mental illness. Coping styles often predict how well a person is able to overcome stressors [24]. Avoidant coping, or emotion-focused coping, is a more passive coping style, which has been shown to result in higher symptoms of depression and anxiety [24, 25]. In contrast, active, or problem-focused, coping has been shown to increase psychological well-being in the face of stressors [25-27]. Figueroa et al. [28] found that psychological well-being in the face of stressors, such as poverty, was predicted by use of problem-focused coping rather than emotion-focused coping.

Despite well-documented positive effects of problem-focused coping, women tend to engage more in avoidant, or emotion-focused, coping styles. This can often lead to higher psychological distress in women [29], though adult women are not the only ones who engage in emotion-focused coping. In their study of adolescent girls, Broderick and Korteland [30] found that girls engaged more often in emotionfocused coping than did boys and thus were more likely to develop depressive symptoms. This finding indicates that emotion-focused coping in women may be cultivated from a young age. 
Few studies have explored why these gender differences exist in coping styles, though there is evidence that links gender differences in coping styles to gendered beliefs. Broderick and Koreland [30] found that the more adolescent girls believed in traditional gender roles, the more likely they were to engage in passive coping styles. In their analysis of coping and gender, Banyard and Graham-Bermann [31] argued that societal factors, such as power differences between men and women, affected the way in which women cope with stress. Gendered beliefs and power differences are not inherent in women; they are developed through the way society treats women. From a feminist theoretical perspective, one can see how gendered beliefs and a sense of powerlessness promote passive coping styles in women. In the context of Objectification Theory, it is possible to attribute this to the objectification of women. When women are objectified, their identity and worth are defined by others [15], stripping women of their most basic power, the ability to define one's own identity and worth. It is possible that, through this process (the loss of basic power and agency), women learn to become passive and thus engage more frequently in passive coping.

\subsection{Hypotheses}

The present study examined the relationships between positive sexual awareness, sexual self-monitoring, coping, and psychopathological symptoms in a college student sample. Using the Sexual Awareness Questionnaire [32], we hypothesized that positive sexual awareness would be positively related to adaptive coping and negatively related to symptoms of psychopathology, while sexual self-monitoring, indicative of being objectified, would be positively related to avoidant, passive coping and symptoms of psychopathology. Analyses also tested whether the relationships between sexual self-monitoring and symptoms of psychopathology were mediated by avoidant coping. Given the greater salience of objectification for women than men, it was also hypothesized that the above relationships would be stronger for women than for men.

\section{Methods}

\subsection{Participants}

Four hundred thirteen undergraduate psychology students (283 female, 127 male, 1 other, 2 not reported) at a large southwestern state university completed an anonymous online survey for research participation credit. The majority of the participants identified as straight (90.1\%), followed by bisexual (5.8\%), gay or lesbian $(2.7 \%)$, and other $(1.2 \%)$. The mean age of the sample was 19.4 years $(\mathrm{SD}=2.7)$ for women and 20.0 years $(\mathrm{SD}=3.0)$ for men. The race/ethnicity of the sample was 38.0\% Caucasian/White, 26.6\% Hispanic/Latino, 20.1\% Asian/Pacific Islander, 11.1\% African American/Black, and 3.6\% other. Almost half of the participants (45.4\%) defined themselves as not currently dating, followed by steady or exclusive daters at $38.3 \%$, occasionally dating at $10.9 \%$, married $2.4 \%$, and engaged $2.7 \%$.

\subsection{Measures}

Reported Cronbach's alphas are based on the present sample.

Somatization was measured by the Patient Health Questionnaire PHQ-15

Somatic Symptom Severity scale [33,34] ( $\alpha=0.77)$, which consists of 15 questions asking about how often one has been bothered by somatization symptoms, such as 
"stomach pain" and "dizziness," in the past 2 weeks. Responses are coded $0=$ "Not Bothered," 1 = "Bothered A Little," and 2 = "Bothered A Lot." Scores are the sum of the responses across the 15 items.

Depression was measured by the Patient Health Questionnaire PHQ-9

Depression scale [35] ( $\alpha=0.85)$, which consists of 9 questions asking about the frequency of depressive symptoms one has experienced in the past 2 weeks, with items such as "Little interest or pleasure in doing things" and "Feeling down, depressed, or hopeless." Responses are coded 0 = "Not at all," 1 = "Several days," 2 = "More than half the days," and 3 = "Nearly every day." Scores are the sum of the responses across the 9 items.

Anxiety was measured by the Patient Health Questionnaire GAD-7 Anxiety scale [36] ( $\alpha=0.82)$, which consists of 7 questions asking about the frequency of anxiety symptoms one has experienced in the past 4 weeks, with items such as "Feeling nervous, anxious, on edge, or worrying a lot about different things." Responses are coded 0 = "Not at all," 1 = "Several days," 2 = "More than half the days," and 3 = "Nearly every day." Scores are the sum of the responses across the 7 items. Since the depression and anxiety scales were highly correlated $(r=0.78)$, they were combined (averaged) to form a composite depression-anxiety scale.

Eating disorder symptoms were measured by the Patient Health Questionnaire PHQ-ED Eating Disorder scale [34] $(\alpha=0.70)$, which consists of 8 yes-no questions asking about the occurrence of behaviors indicative of a binge eating disorder. An example item is "Do you often feel that you can't control what or how much you eat?" Scores are the number of yes responses across the 8 items.

Sexual awareness was measured by the Sexual Awareness Questionnaire [32], a 36-item self-report measure designed to measure four personality traits associated with sexual awareness and assertiveness: sexual consciousness (the tendency to think about one's own sexuality; "I am very aware of my sexual feelings"; $\alpha=0.85$ ), sexual monitoring (the awareness one has of the impression one's sexuality makes on others; "I'm concerned about the sexual appearance of my body"; $\alpha=0.76$ ), sexual assertiveness (the tendency to be assertive in one's sexual desires; "I'm assertive about the sexual aspects of my life"; $\alpha=0.80$ ), and sex appeal consciousness ("I am quick to sense whether others think I'm sexy"; $\alpha=0.93$ ). Participants are asked to indicate, on a scale of zero to four, how characteristic of him/her each statement is. Subscale scores are the sum of the responses on the relevant items. Sexual consciousness correlated 0.64 with sexual assertiveness in the present sample, so these 2 scales were combined (averaged) to form a positive sexual awareness scale, in contrast to the sexual self-monitoring scale, which may reflect one's acceptance of being sexually objectified. The 3-item sex appeal consciousness subscale was not used in the present analyses. The Sexual Awareness Questionnaire has been validated on a population of undergraduate students.

Coping was measured by the Brief COPE [37], which consists of 14 two-item subscales that measure different coping responses. For the present study, participants were asked to report on their coping behaviors in the past year (indicative of a "coping style") using a 4-point response scale ranging from 1 = "I haven't been doing this at all" to 4 = "I've been doing this a lot." Six of the subscales (active coping, emotional support, instrumental support, positive reframing, planning, acceptance) were combined (averaged) to form an adaptive coping scale $(\alpha=0.82)$. Three of the subscales (denial, substance use, behavioral disengagement) were combined to form an avoidant coping scale $(\alpha=0.73)$. While Carver [37] states that the separate COPE subscales are not meant to be combined into necessarily adaptive vs. maladaptive avoidant coping, the above schema made sense in terms of the idea of agency discussed above, and the alphas for the composite scales indicate that the separate COPE subscales did cluster within the composite scales. 


\section{Results}

Independent sample t-tests compared sex differences in somatization, depression-anxiety, eating disorder symptoms, positive sexual awareness, sexual self-monitoring, and adaptive and avoidant coping (Table 1). Women scored significantly higher than men on somatization and depression-anxiety, while men scored higher than women on both sexual consciousness/assertiveness and sexual monitoring.

Table 2 presents the correlations among somatization, depression-anxiety, eating disorder symptoms, sexual consciousness/assertiveness, sexual self-monitoring, and adaptive and avoidant coping separately by gender. For both women and men, somatization, depression-anxiety, and eating disorder symptoms were significantly positively correlated with each other. Contrary to hypotheses, however, these disorders were not significantly negatively correlated with positive sexual awareness (consciousness + assertiveness). These disorder symptoms were, on the other hand, positively correlated

\begin{tabular}{|c|c|c|c|}
\hline & \multicolumn{2}{|c|}{ Gender } & \multirow[t]{3}{*}{$t$} \\
\hline & Female & Male & \\
\hline & $M(S D)$ & $M(S D)$ & \\
\hline Somatization & $6.37(3.94)$ & $3.88(3.55)$ & $-5.71^{* *}$ \\
\hline Depression-anxiety & $6.29(4.22)$ & $5.37(3.87)$ & $-2.02^{*}$ \\
\hline Eating disorder & $0.89(1.40)$ & $0.87(1.33)$ & -0.14 \\
\hline Positive sexual awareness & $13.78(5.47)$ & $15.22(4.88)$ & $2.34^{*}$ \\
\hline Sexual monitoring & $17.50(7.25)$ & $19.26(6.28)$ & $2.26^{*}$ \\
\hline Adaptive coping & $2.83(0.62)$ & $2.74(0.67)$ & $2.34^{*}$ \\
\hline Avoidant coping & $1.55(0.63)$ & $1.60(0.74)$ & $2.56^{*}$ \\
\hline $\begin{array}{l}<0.05 \\
<0.001\end{array}$ & & & \\
\hline
\end{tabular}

Table 1.

Means and standard deviations by gender.

\begin{tabular}{|c|c|c|c|c|c|c|c|}
\hline & Som. & $\begin{array}{l}\text { Dep.- } \\
\text { anx. }\end{array}$ & ED & $\begin{array}{l}\text { Pos. } \\
\text { sex. } \\
\text { aware. }\end{array}$ & $\begin{array}{l}\text { Sex. } \\
\text { mon. }\end{array}$ & $\begin{array}{l}\text { Adapt. } \\
\text { coping }\end{array}$ & $\begin{array}{l}\text { Avoid. } \\
\text { coping }\end{array}$ \\
\hline Somatization & & $0.53^{* * *}$ & $0.19^{* *}$ & 0.04 & $0.14^{*}$ & 0.04 & $0.31^{* * *}$ \\
\hline $\begin{array}{l}\text { Depression- } \\
\text { anxiety }\end{array}$ & $0.60^{* * *}$ & & $0.28^{* * *}$ & 0.00 & $0.17^{* *}$ & -0.02 & $0.41^{* * *}$ \\
\hline Eating disorder & $0.30^{* *}$ & $0.31^{* * *}$ & & 0.02 & 0.12 & -0.03 & $0.37^{* * *}$ \\
\hline $\begin{array}{l}\text { Positive sexual } \\
\text { awareness }\end{array}$ & 0.05 & -0.07 & 0.01 & & $0.20^{* * *}$ & $0.15^{*}$ & -0.01 \\
\hline $\begin{array}{l}\text { Sexual } \\
\text { monitoring }\end{array}$ & 0.17 & 0.05 & 0.13 & $0.33^{* * *}$ & & 0.08 & $0.19^{* *}$ \\
\hline Adaptive coping & $0.20^{*}$ & 0.08 & -0.03 & $0.32^{* * *}$ & 0.17 & & $0.14^{*}$ \\
\hline Avoidant coping & 0.18 & 0.14 & $0.28^{* *}$ & 0.04 & 0.13 & $0.38^{* * *}$ & \\
\hline \multicolumn{8}{|c|}{$\begin{array}{l}{ }^{a} \text { Women above the diagonal and men below the diagonal. } \\
{ }^{*} p<0.05 . \\
{ }^{* *} p<0.01 . \\
p<0.001 .\end{array}$} \\
\hline
\end{tabular}

Table 2.

Correlations among variables ${ }^{a}$. 
with sexual self-monitoring for men and women, with these correlations reaching statistical significance for women. As expected, for both women and men, positive sexual awareness was significantly positively correlated with adaptive coping, while sexual self-monitoring was significantly positively correlated with avoidant coping for women. The one notable gender difference in the correlations was the higher correlations of avoidant coping with somatization, depression-anxiety, and eating disorder symptoms for women than for men, with this difference reaching statistical significance for depression-anxiety (Fisher's $z$-test $=-2.73, p<0.01$ ).

The patterns of correlations suggested that avoidant coping might be significantly mediating the relationships between sexual monitoring and psychopathology particularly in women. To test this, a path analysis (Figures 1 and 2) was conducted that modeled positive sexual awareness and sexual self-monitoring as exogenous variables predicting adaptive and avoidant coping that, in turn, were tested as predictors of somatization, depression-anxiety, and eating disorder symptoms. Combined and separate analyses were also run for sex, with a difference chi-square calculated for the combined analyses to test for the equivalence of the estimated paths for the male versus female matrices. Mediation effects were tested using the

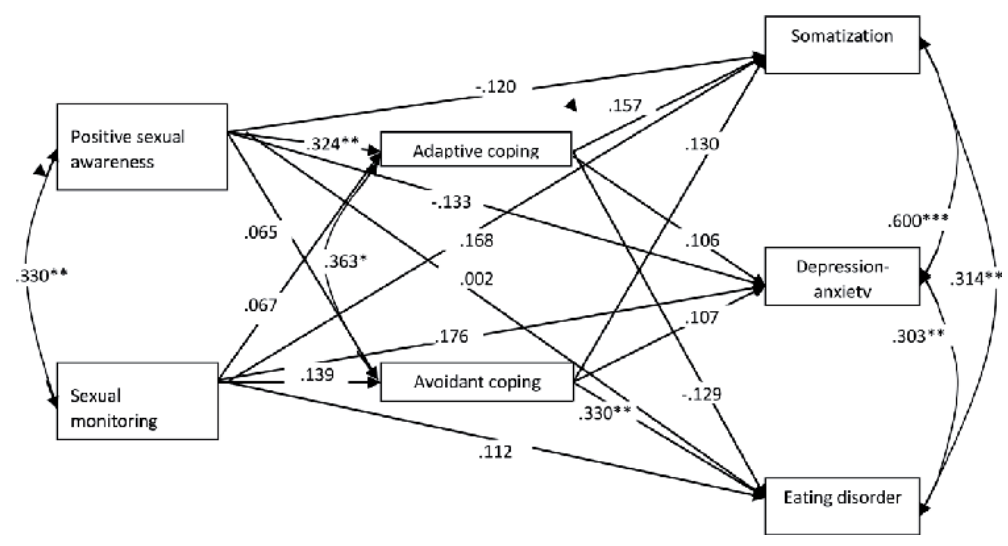

Figure 1.

Path diagram showing standardized estimates for the model testing direct and indirect pathways between positive sexual awareness, sexual monitoring, coping, and psychological maladjustment: Men. $\mathrm{p}<0.05$, $\mathrm{p}<0.01,{ }^{* * *} \mathrm{p}<0.001$

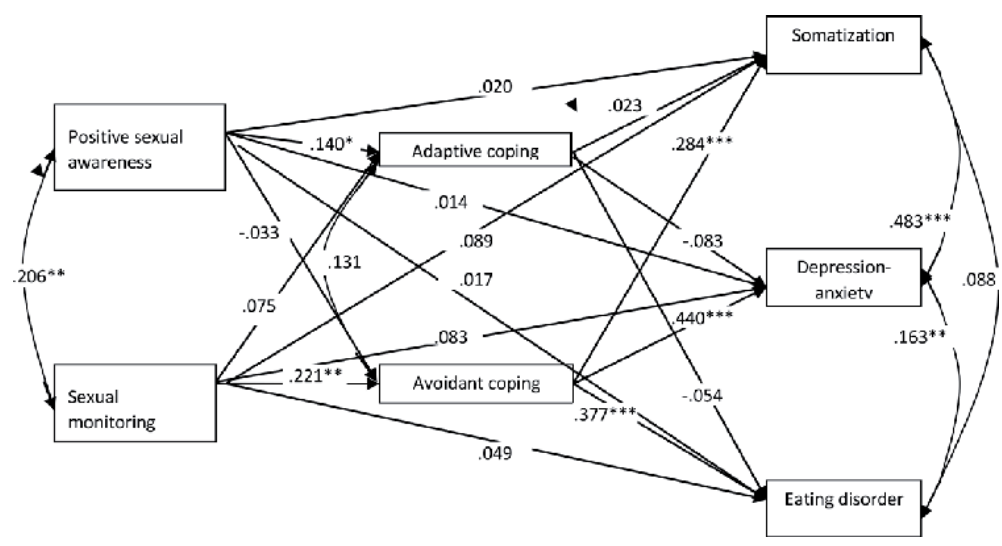

Figure 2.

Path diagram showing standardized estimates for the model testing direct and indirect pathways between positive sexual awareness, sexual monitoring, coping, and psychological maladjustment: Women. ${ }_{* * *}^{*}<0.05$, $\mathrm{p}<0.01, \quad \mathrm{p}<0.001$. 
bias corrected bootstrapping of confidence intervals option in Mplus version 5.21 [38] with maximum likelihood estimation using the covariance matrix. Missing data were handled using full information maximum likelihood.

Results of the path analyses are presented separately for the male and female estimates, as the difference chi-square for this model approached significance $\left(\chi^{2}=30.267,21 \mathrm{df}, p=0.087, \mathrm{CFI}=0.967\right.$, RMSEA $\left.=0.047\right)$, and there was a theoretical reason for expecting gender differences in the pathways. It is notable, that while positive sexual awareness was significantly positively predictive of adaptive coping for men and women, sexual self-monitoring was only significantly predictive of avoidant coping in women. Controlling for sexual monitoring, avoidant coping was a significant predictor of somatization, depression-anxiety, and eating disorder symptoms for women and of eating disorder symptoms in men. The direct paths from sexual self-monitoring to psychopathology were not significant for women or men. Bootstrapped mediation analyses indicated that for men the relationships between sexual self-monitoring and psychopathology were not significantly mediated by avoidant coping ( $95 \%$ confidence interval: $-0.010,0.063$ for somatization; $-0.008,0.060$ for depression-anxiety; $-0.004,0.033$ for eating disorders). In contrast, all of these mediated pathways were significant for women at the 0.01 significance level (99\% confidence interval, 0.004, 0.085 for somatization; 0.011, 0.134 for depression-anxiety; $0.004,0.037$ for eating disorders). None of the indirect paths involving positive sexual awareness or adaptive coping were significant.

\section{Discussion}

This is the first study to differentiate the effects of positive vs. negative aspects of sexual awareness/consciousness on coping and psychological functioning. While men scored higher than women on both positive sexual awareness (sexual consciousness and sexual assertiveness) and sexual self-monitoring, the patterns of correlations and path analysis results suggest that sexual self-monitoring has different meanings and implications for men vs. women. A study by Gillen et al. [39], in fact, suggests that such sexual self-consciousness may reinforce traditional gender beliefs about sexuality. As expected, positive sexual awareness was associated with greater adaptive coping in men and women, but sexual self-monitoring was more associated with avoidant coping in women than men. In support of Objectification Theory, sexual self-monitoring and avoidant coping were more predictive of psychopathological symptoms in women than men, and the relationships between sexual self-monitoring and psychopathological symptoms were significantly mediated by avoidant coping only in women. These results indicate that women who score high in sexual self-monitoring tend to engage in avoidant coping styles, which in turn contributes to higher rates of reported symptoms of depression, anxiety, somatization, and disordered eating. In contrast, women who scored high on sexual consciousness/assertiveness were found to engage in more active coping styles, although no mediation relationship was found between this and symptoms of mental illness. In the context of Objectification Theory, these results are to be expected. Sexual self-monitoring, which indicates a person's fixation on his/her attractiveness to others, can serve as an indicator of sexual objectification, as women who experience objectification often become fixated on their body and attractiveness to others [19].

The implications for this study are two-fold. First, this study supports the idea that the sexual objectification of women contributes to poor mental health outcomes by taking away their sense of agency. Second, this study suggests that therapeutic interventions which incorporate agency may be beneficial for work with women, including perhaps those who have suffered some form of sexual violence. 


\subsection{Limitations and future directions}

While the sample was recruited from an ethnically diverse population, all participants were college students from a university in a particular region of the U.S., which limits generalizability. The cross-sectional nature of the research also limits causal inference. Future research should focus on how experiences of objectification, especially in women, lead to maladaptive coping behaviors and increased risk of psychopathology. In addition to positive and negative sexual awareness, future studies should directly measure self-perceived self-objectification [40]. Research on therapeutic practices with women should consider ways in which sexual and gender empowerment may produce salutary effects through increasing adaptive coping behaviors.

Future studies of objectification should also focus on the other forms objectification takes, such as treating a person as a servant or otherwise denying their humanity [41]. This is prevalent in cases of domestic violence, where an abusive partner abuses in order to maintain power and control over his partner, so that he/ she remains focused on meeting the abuser's needs [42]. This study also calls for an exploration of objectification and its effects on coping and psychological maladjustment in other populations. Members of racial/ethnic minorities in the U.S. often experience the objectification of their bodies [43], and children who suffer childhood abuse may also have parents who view them as objects [44].

Respect for the autonomy of others is at the core of many moral philosophies. Martin Buber [45] explicitly distinguishes between I-thou relationships between autonomous conscious beings versus I-it relationships between such beings and inanimate objects. I-thou relationships require a moral consciousness, while treating a person as if they were an object is inherently immoral. Meanwhile, psychologists [46] have recognized the role of autonomy in enabling moral consciousness and behavior. The present study shows how the objectification of women in the context of their sexuality not only strips women of their autonomy, with consequent deleterious effects on their psychological functioning, but is also inherently immoral. Beyond working to achieve political and economic gender equality, public policies and educational practices should also work to encourage women to be empowered in their sexuality rather than objects in a male-controlled world.

\section{Author details}

Catherine Baggett ${ }^{1}$, Craig Nagoshi ${ }^{1 *}$ and Julie Nagoshi ${ }^{2}$

1 University of Texas at Arlington, Arlington, Texas, USA

2 Arizona State University, Phoenix, Arizona, USA

*Address all correspondence to: cnagoshi@uta.edu

\section{IntechOpen}

(C) 2020 The Author(s). Licensee IntechOpen. This chapter is distributed under the terms of the Creative Commons Attribution License (http://creativecommons.org/licenses/ by/3.0), which permits unrestricted use, distribution, and reproduction in any medium, provided the original work is properly cited. (cc) BY 


\section{References}

[1] American Psychiatric Association. Diagnostic and Statistical Manual of Mental Disorders. 5th ed. Washington, D.C: American Psychiatric Association; 2015

[2] McLean CP, Asnaani A, Litz BT, Hofmann SG. Gender differences in anxiety disorders: Prevalence, course of illness, comorbidity and burden of illness. Journal of Psychiatric Research. 2011;45:1027-1035. DOI: 10.1016/j. jpsychires.2011.03.006

[3] Tolin DF, Foa EB. Sex differences in trauma and posttraumatic stress disorder: A quantitative review of 25 years of research. Psychological Bulletin. 2006;132:959-992. DOI: 10.1037/0033-2909.132.6.959

[4] Smith SG, Chen J, Basile KC, Gilbert LK, Merrick MT, Patel N, et al. The National Intimate Partner and Sexual Violence Survey (NISVS): 20102012 State Report. Atlanta, GA: National Center for Injury Prevention and Control, Centers for Disease Control and Prevention; 2017

[5] Valenti J. The Purity Myth: How America's Obsession with Virginity is Hurting Young Women. Berkeley, CA: Seal Press; 2011

[6] Rosen R, Bachmann G. Sexual wellbeing, happiness, and satisfaction, in women: The case for a new conceptual paradigm. Journal of Sex and Marital Therapy. 2008;34:291-297. DOI: 10.1080/00926230802096234

[7] Holmberg D, Blair KL, Phillips M. Women's sexual satisfaction as a predictor of well-being in same-sex versus mixed-sex relationships. Journal of Sex Research. 2010;47:1-11. DOI: $10.1080 / 00224490902898710$

[8] Donaghue N. Body satisfaction, sexual self-schemas and subjective well-being in women. Body Image. 2009;6:37-42. DOI: 10.1016/j. bodyim.2008.08.002

[9] Stephenson KR, Meston CM. The conditional importance of sex: Exploring the association between sexual well-being and life satisfaction. Journal of Sex and Marital Therapy. 2015;41:25-38. DOI: 10.1080/0092623X.2013.811450

[10] Carrobles JA, Gámez Guadix M, Almendros C. Funcionamiento sexual, satisfacción sexual y bienestar psicológico y subjetivo en una muestra de mujeres españolas. Anales de Psicología. 2011;27:27-34

[11] Laumann EO, Paik A, Glasser DB, Kang J, Wang T, Levinson B, et al. A cross-national study of subjective sexual well-being among older women and men: Findings from the global study of sexual attitudes and behaviors. Archives of Sexual Behavior. 2006;35:143-159. DOI: $10.1007 / \mathrm{s} 10508-005-9005-3$

[12] Owen J, Fincham FD, Moore J. Short-term prospective study of hooking up among college students. Archives of Sexual Behavior. 2011;40:331-341. DOI: $10.1007 /$ s10508-010-9697-x

[13] Wiederman MW. Women's body image self-consciousness during physical intimacy with a partner. Journal of Sex Research. 2000;37:60-68. DOI: 10.1080/00224490009552021

[14] Wiederman MW. Body image and sexual functioning. In: Cash TF, editor. Encyclopedia of Body Image and Human Appearance. Vol. 1. San Diego: Academic Press; 2012. pp. 148-152

[15] Fredrickson BL, Roberts T. Objectification theory: Toward understanding women's lived experiences and mental health risks. 
Psychology of Women Quarterly. 1997;21:173-206. DOI: 10.1111/j.14716402.1997.tb00108.x

[16] Ramsey LR, Hoyt T. The object of desire: How being objectified creates sexual pressure for women in heterosexual relationships. Psychology of Women Quarterly. 2015;39:151-170. DOI: $10.1177 / 0361684314544679$

[17] Register JD, Katrevich AV, Aruguete MS, Edman JL. Effects of self-objectification on self-reported eating pathology and depression. The American Journal of Psychology. 2015;128:107-113. DOI: 10.5406/ amerjpsyc.128.1.0107

[18] Tiggemann M, Slater A. The role of self-objectification in the mental health of early adolescent girls: Predictors and consequences. Journal of Pediatric Psychology. 2015;40:704-711. DOI: 10.1093/jpepsy/jsv021

[19] Jongenelis MI, Byrne SM, Pettigrew S. Self-objectification, body image disturbance, and eating disorder symptoms in young Australian children. Body Image. 2014;11:290-302. DOI: 10.1016/j.bodyim.2014.04.002

[20] Calogero RM, Davis WN, Thompson JK. The role of selfobjectification in the experience of women with eating disorders. Sex Roles. 2005;52:43-50. DOI: 10.1007/ s11199-005-1192-9

[21] Tiggemann M, Williams E. The role of self-objectification in disordered eating, depressed mood, and sexual functioning among women: A comprehensive test of objectification theory. Psychology of Women Quarterly. 2012;36:66-75. DOI: $10.1177 / 0361684311420250$

[22] Szymanski DM, Henning SL. The role of self-objectification in women's depression: A test of objectification theory. Sex Roles. 2007;56:45-53. DOI: 10.1007/s11199-006-9147-3
[23] Carr ER, Green B, Ponce AN. Women and the experience of serious mental illness and sexual objectification: Multicultural feminist theoretical frameworks and therapy recommendations. Women and Therapy. 2015;38:53-76. DOI: 10.1080/02703149.2014.978216

[24] Roth S, Cohen LJ. Approach, avoidance, and coping with stress. The American Psychologist. 1986;41:813-819

[25] Foyal LW. The relationship between coping and depressed mood: The impact of coping strategy and situational control [doctoral dissertation]. Greensboro, NC: The University of North Carolina at Greensboro; 1998

[26] Thompson RJ, Mata J, Jaeggi SM, Buschkuehl M, Jonides J, Gotlib IH. Maladaptive coping, adaptive coping, and depressive symptoms: Variations across age and depressive state. Behavior Research and Therapy. 2010;48:459-466. DOI: 10.1016/j. brat.2010.01.007

[27] O'Connor DB, Shimizu M. Sense of personal control, stress and coping style: A cross-culutral study. Stress and Health. 2002;18:173-183. DOI: 10.1002/ smi.939

[28] Figueroa MI, Contini N, Lacunza AB, Levin M, Suedan AE. Las estrategias de afrontamiento y su relación con el nivel de bienestar psicológico: Un estudio con adolescentes de nivel socioeconómico bajo de Tucumán (Argentina). Anales de Psicología. 2005;21:66-72

[29] Matud MP. Gender differences in stress and coping styles. Personality and Individual Differences. 2004;37:14011415. DOI: 10.1016/j.paid.2004.01.010

[30] Broderick PC, Korteland C. Coping style and depression in early adolescence: Relationships to gender, gender role, and implicit beliefs. 
Sex Roles. 2002;46:201-213. DOI: 10.1023/A:1019946714220

[31] Banyard VL, Graham-Bermann SA. Can women cope? A gender analysis of theories of coping with stress. Psychology of Women Quarterly. 1993;17:303-318

[32] Snell WE Jr, Fisher TD, Miller RS. Development of the sexual awareness questionnaire: Components, reliability, and validity. Sexual Abuse. 1991;4:65-92. DOI: 10.1177/107906329100400104

[33] Kroenke K, Spitzer RL, Williams JBW. The PHQ-15: Validity of a new measure for evaluating somatic symptom severity. Psychosomatic Medicine. 2002;64:258-266. PMID: 11914441

[34] Spitzer RL, Kroenke K, Williams JB. Validation and utility of a self-report version of PRIME-MD: The PHQ primary care study. JAMA: The Journal of the American Medical Association. 1999;282:1737-1744. PMID: 10568646

[35] Kroenke K, Spitzer RL. The PHQ-9: A new depression diagnostic and severity measure. Psychiatric Annals. 2002;3:509-521. DOI: 10.3928/0048-5713-20020901-06

[36] Spitzer RL, Kroenke K, Williams JBW, Löwe B. A brief measure for assessing generalized anxiety disorder: The GAD-7. Archives of Internal Medicine. 2006;166:1092-1097. DOI: 10.1001/archinte.166.10.1092

[37] Carver CS. You want to measure coping but your protocol's too long: Consider the brief COPE. International Journal of Behavioral Medicine. 1997;4:92-100. DOI: 10.1207/ s15327558ijbm0401_6

[38] Muthén LK, Muthén BO. Mplus Software Program. 5th ed. Los Angeles, CA: Muthén \& Muthén; 1998-2007
[39] Gillen MM, Lefkowitz ES, Shearer CL. Does body image play a role in risky sexual behavior and attitudes? Journal of Youth and Adolescence. 2006;35:243-255. DOI: $10.1007 /$ s10964-005-9005-6

[40] Talmon A, Ginzburg K. The nullifying experience of selfobjectification: The development and psychometric evaluation of the selfobjectification scale. Child Abuse and Neglect. 2016;60:46-57. DOI: 10.1016/j. chiabu.2016.09.007

[41] Papadaki L. What is objectification? Journal of Moral Philosophy. 2010;7:1636. DOI: $10.1163 / 174046809 X 12544019$ 606067

[42] Langhinrichsen-Rohling J, McCullars A, Misra T. Motivations for men and women's intimate partner violence perpetration: A comprehensive review. Partner Abuse. 2012;3:429-468. DOI: 10.1891/1946-6560.3.4.429

[43] Collins PH. Black Sexual Politics: African Americans, Gender, and the New Racism. New York: Routledge; 2005

[44] American Psychological Association. Report of the APA Task Force on the Sexualization of Girls. 2007. Available from: www.apa.org/ pi/wpo/sexualization.html. [Accessed: December 13, 2019]

[45] Buber M. I and Thou. New York: Charles Scribner's Sons; 1937

[46] Hogan R. Moral conduct and moral character: A psychological perspective. Psychological Bulletin. 1973;79:217-232 


\title{
Chapter 6
}

\section{Political Gender Gap and Social Dominance Orientation}

\author{
Minou Ella Mebane, Antonio Aiello and Donata Francescato
}

\begin{abstract}
In the last 30 years, there was a rise in the political gender gap. The Center for the American Woman and Politics data shows that a larger proportion of women than men vote for the Democratic Party. Women tend to differ from men in several political issues endorsing more welfare policies and progressive policy changes and are less racist than men. Social dominance orientation (SDO) has been theorized to account for political gender differences. Men have higher average levels of SDO than women, everything else being equal. To test the gender invariance hypothesis, we believe it's important to take into consideration people who not only identify with groups that hold specific hierarchy-enhancing or hierarchy-attenuating ideologies but who participate in them promoting their values and ideas. In this chapter, we describe the findings of research on gender differences in SDO of activists in political parties that range from extreme left-wing to extreme right-wing.
\end{abstract}

Keywords: political decisions, elections, gender gap, social dominance, political

\section{Introduction}

The increase of participation of women in politics has revealed that women and men are political actors with distinct political preferences $[1,2]$. In the last 30 years, there was a rise in the political gender gap. The Center for the American Woman and Politics [1] data show that a more substantial proportion of women than men vote for the Democratic Party. The last presidential elections (2016) revealed a sizable 11 percentage-point gender gap, 42 percent of women voted for Trump versus 53 percent of men [1]. This is not a new phenomenon according to the data of CAWP in the last two decades, since in the 1996 presidential election, women voters tend to prefer more a democratic candidate than men (the gender gap variance has varied in these years from a minimum of 7 points to a maximum of 11 points). The 2016 gender gap was one of the largest ones. Also, in Europe, most countries show either no gender gap or that women are more left-wing than men. Recent research [3] based on the analysis of the European Values Study/World Values Survey that combines data spanning from 1989 to 2014 reveals that there is a gender-generation gap. In the younger cohorts, women are more left-wing oriented than men.

Researches on the gender difference in political issues point out that there is a wide difference in programs and issues that women and men support. Women, in general, are in favor of government spending on social welfare, education, and health. They are more likely to favor programs for medical care, schooling, and gun control.

On the contrary, they tend to oppose more military spending or the use of force to solve conflicts and are against capital punishment [4-6]. They support less 
discriminatory policies and have more positive attitudes toward homosexuals than men [7]. Women have lower levels of prejudice [8], authoritarianism [9, 10], and anti-egalitarianism, [11] are more worried about potential international conflicts [12], and, in general, hold less punitive attitudes [11]. Social dominance orientation (SDO) has been theorized to account for political gender differences [13-15].

\section{Social dominance orientation}

The social dominance theory (STD) aims to understand how group-based social hierarchy is formed and preserved [13]. According to Sidanius and Pratto [13], postindustrial societies tend to develop group-oriented social hierarchies that support long-term human survival. In these hierarchical societies, intergroup conflicts and oppressions contribute to maintaining the status quo of the social system. SDT suggests that an individual orientation called social dominance orientation $[13,16,17]$ is a potential explanatory factor of sociopolitical sex differences. The SDO has been defined as a personal desire for group-based dominance, mirroring an individual's support for group-based hierarchies [13]. People higher in SDO tend to support hierarchy-enhancing legitimizing myths such as prejudice, racism, sexism, militarism, support for the death penalty, and coercive social power across societies and contexts [16,18-22]. Men tend to score higher than women in SDO [13]. Such differences may be, to a certain extent, determined by the desire of males to justify their dominant position in society. As Sidanius and Pratto [13] point out, our contemporary hierarchical system is mostly "andrarchical” since men tend still occupy most of the highest positions of political and economic power. Therefore, men should support social systems that maintain hierarchies since they tend to hold privileges due to occupying higher positions in society.

In our societies, women and men usually have different roles in the group-based hierarchy. Men tend to be more numerous in the police, military, lawyers, judges, and business executives areas, whereas women are in a more significant number in the teachers, social workers, and charity volunteer areas [23]. Overall, men are inclined to participate in institutions or hold roles that enhance hierarchy and females on the contrary to institutions that diminish hierarchy [24]. The SDT maintains that those that occupy positions in society that reinforce the existing group inequality or strengthen in-group status are more likely to be social dominanceoriented than out-groups are.

SDT claims that men and women should exhibit differences in SDO due to strategies that follow from evolutionary theory. Sidanius et al. [25] maintain that different psychological and behavioral predispositions between males and females in terms of sexual and reproductive behavior are the core of gender difference in society. From this perspective, sex differences in orientation toward group-based social inequality (SDO) are the effects of human reproductive strategies. Sidanius and Pratto [13] put forward that reproductive inequality implies economic inequality and economic inequality implies political inequality. Sidanius, Pratto, and Bobo [25] formulate the gender invariance hypothesis from a perspective of theoretical biocultural interaction: "Not only should men have a higher average level of SDO, but this higher average level of male SDO should also be found after cultural, situational and environmental factors are considered" (p. 1000) [25].

Two invariance hypotheses have been proposed. The "strong" version and the "soft" one. The strong version claims that SDO differences between men and women should not vary across cultural factors, situational factors, or both. There should, therefore, be no significant interaction between sex gender and culturalsituational factors. In other words, the strong version of the invariance hypothesis 
predicts that the difference in SDO between men and women should be essentially invariant across all major cultural, environmental, and situational factors such as country of national origin, ethnicity, education, income, age, political ideology, racism, religious beliefs, and gender role attitudes. However, since the claim of the biological roots of gender differences is less relevant in SDT today [18], a contextual variation is, to some extent, allowed in the soft version hypothesis [26]. The soft version of the invariance hypothesis asserts that men will always show higher levels of SDO than women, everything else being equal. It claims that although gender might interact with several cultural-situational factors, this interaction will always be ordinal and never disordinal. Whereas the male-female differences in SDO might show some significant variations across cultural factors, situational factors, or both, females should never have significantly higher SDO than males within the same sociocultural context [25]. Both socialization experiences and belonging to hierarchy-attenuating or hierarchy-enhancing settings can increase or diminish SDO [27]; however, women should never have a significantly higher SDO than men. For instance, different professional groups may vary in their levels of SDO [20]; however, within a specific professional group, men should report higher levels of SDO than women [28].

Quite a lot of studies have attempted to investigate, if and under which, circumstances the invariance hypothesis holds. Several studies conducted mainly by Sidanius and colleagues supported the validity of the invariance hypothesis, both with samples of students and adult residents of the United States and in many foreign countries [13-15, 24, 25, 29, 30].

For example, in their cross-cultural study on male-female difference in SDO that involved 10 countries (Australia, Canada, Israel, Mexico, Palestine, Republic of China, New Zealand, the former USSR, Sweden, and the United States), Sidanius and Pratto showed that males are significantly more social dominance-oriented than females in 39 of the 45 samples [13].

Also, Wilson and White [31] in their study based on students and adults revealed that males were more social dominant and politically conservative than women. Social dominance mediated the relationship between gender and conservatism.

Furthermore, studies confirm that even in countries that traditionally promote gender equality, the gender gap in social dominance orientation prevails [32, 33].

Contrary evidence emerged, however, in other studies. Research based on student and adult samples from Australia, the United States, Ireland, and Sweden did not confirm the main gender effect [34-38].

In Taiwan, females scored higher than males, but the difference was not significant [39], and in two samples in Israel and Australia, men did not score significantly higher than females [34, 39]. In Küpper and Zick's [40] first study, women unexpectedly showed higher levels of SDO than men.

Some studies on the gender invariance hypothesis investigated whether group differences in SDO can be explained by group identification.

Wilson and Liu [41], following the social identity theory (SIT) perspective, predicted that males who identify strongly with gender group should exhibit higher SDO scores than low-identifying males and that females who identify strongly with their gender group should score lower than low-identifying females. Their findings showed that the gender-SDO relationship was moderated by the strength of gender in-group identification: increasing group identification was associated with decreasing SDO scores for females and increasing SDO scores for females. Sidanius and Pratto [28], however, criticized this study for not meeting the criteria, "all else being equal in principle." They underlined that they should have compared men and women with similar levels of gender identification. 
Also, Huanga and Liu [42] analyzed the controversy in the literature concerning whether group differences in SDO can be explained by group identification. They hypothesized that if SDO acts as a stable individual difference, it should maintain its relative relationship with gender (i.e., men should have higher SDO than women) even when the demographic group is saliently primed. Alternatively, from a situational priming perspective, one might expect gender differences in SDO to be significant only when gender is salient. Their first research involved 1605 adults in Taiwan, and they found that contrary to SDT's invariance hypothesis, men were higher on SDO than women only when gender was salient.

Foels and Pappas [43] tested the invariance hypothesis by measuring the relationship between sex and SDO while controlling for the effects of gender socialization. They demonstrated that the sex difference in SDO is mediated by gender socialization.

Lee et al. [33] addressed the dispute between SDT and social identity theory (SIT) in a meta-analysis. Their research showed that in what has been predicted by SDO, gender differences on SDO were more substantial and more stable than differences between ethical and racial groups in the United States and worldwide.

Other studies on the gender invariance hypothesis explored the influence of various kinds of presumed hierarchy-enhancing or hierarchy-attenuating settings. Several studies have shown that university majors and career choices are associated with either hierarchy-enhancing (HE, e.g., racism) or hierarchy-attenuating (HA, e.g., human rights) legitimizing myths [44]. Dambrun et al. [45] examined the impact of HE vs. HA academic major on stereotyping. They found that students in psychology were less social dominance-oriented than students in law. Moreover, while males were more social dominance-oriented than females in law, no sex difference was found for psychology majors. Authors conclude that their results "can be taken to suggest that social-cultural variables may affect scores on SDO and modify gender differences on SDO" (p. 130). They also notice that female law students had higher SDO scores than male psychology students; this finding is in opposition to the strong version of the invariance hypothesis that men should always score higher than females in SDO.

Sidanius et al. [30] showed conflicting results. In their longitudinal study, they measured the SDO of men and women once a year for the 4- and half-year period. Their findings show that even after controlling for the characteristics of students' academic majors (hierarchy-enhancing or hierarchy-attenuating), males showed significantly higher SDO scores than females did, across the entire college career.

Research, based in Sweden, on gender differences in SDO in social structures varying in equality enhancement and gender composition revealed a main effect of gender on SDO despite the degree of political equality or gender composition. There was an interaction effect only in associations where women were the majority of members [26].

Bathalka et al., [46] investigated the gender invariance hypothesis in similar cultural, ideological, and status contexts. Their findings revealed either no effect for gender or an interaction between gender and the relevant social context and only a small effect size of gender. Overall the authors underline that their results disconfirm the gender invariance assumption of SDT. In their second study, students were categorized according to disciplines HA or HE (literature, languages, psychology, social studies, and anthropology majors were grouped as HA and economics, law, and business as HE majors). Their research showed that whereas HE/HA predicted SDO, gender did not.

Reviewing the literature, we find that most, but not all, studies have found significant differences in favor of males in SDO. However, some studies we discussed showed that males' SDO scores changed according to environmental and socialization variables such as being embedded in hierarchy-attenuating environments, not 
identifying strongly with their gender, or living in societies whose cultural values are more egalitarian and less competitive.

\section{Aims}

Most of the studies that found an invariant gender gap involved students or adolescents [40]. To investigate further the possible causes of the increasing political gender gap, we need to conduct studies with members with strong salient group identities, where the influence of adult socialization egalitarian myths may have the opportunity to influence SDO. On this line, studies on the ideological divide may be done involving militants and politicians. With dispositional features such as personality traits and value differences between conservatives and liberals, right-wing and left-wing voters may be present among ordinary citizens or college students [47] but are more pronounced in groups of party activists, extremists, and politicians. Already in the 1960s, McClosky et al. [48] showed the ideological conflicts were much higher between democratic and republican activists and party leaders than among party voters.

To further explore the gender identity hypothesis and the political gender gap, we need to compare people who not only identify with specific hierarchy-enhancing or hierarchy-attenuating ideologies but who participate actively and continuously to political parties or groups which uphold and promote those ideas. Activism in political parties is a matter of choice in modern society. As Huddy [49] underlines, people who choose to be activists in particular political groups already may hold some hierarchy-enhancing or hierarchy-attenuating ideals. However, their SDO may be heightened or lessened by their prolonged exposure and their internalization of legitimizing myths (i.e., according to "soft" gender invariance hypothesis).

SDO would suggest that being committed activists in a hierarchy-attenuating political groups could make individuals identify with the ideals of the groups. Therefore, male members in these groups could have lower SDO scores than males adhering and participating in hierarchy-enhancing political groups. So, we should find the highest scores of SDO in males belonging to right-wing political groups and the lowest in males active in extreme left-wing groups. However, according to SDT, even while absolute levels of SDO may vary across situations, men should still have relatively higher SDO than women within each political group. The predisposition of males to be temperamentally inclined to dominate, even when exposed to substantial and long-term environmental attenuating pressures, will produce nonetheless residual gender differences even among males and females belonging to groups who uphold egalitarian group values.

To understand if the gender gap in politics could be systematically observed within and across the left-wing and right-wing split, we aimed to compare the accuracy in predicting SDO by gender in four well-defined samples of male and female activists belonging to hierarchy-enhancing political groups - center right and extreme right wing oriented groups - vs. belonging to hierarchy attenuating political groups - center left and extreme left wing oriented groups.

As predicted by SDT, we should find higher mean SDO scores among male activists of extreme right-wing, hierarchy-promoting political groups and lower mean SDO scores in males members of extreme left wing, hierarchy-attenuating political groups. However, according to the invariant gender hypothesis of SDT, the difference between males and females within each group should be invariant across groups: the gender divide should be stronger than the political divide.

On the other hand, as more sustainable within a SIT perspective and gender similarities hypothesis [50], we should find no gender differences both in the 
hierarchy-attenuating extreme left-wing political groups and hierarchy-enhancing extreme right-wing political groups. Both settings should reinforce both in male and female members the dominant legitimizing myths of the political group they have chosen, and the political divide should be stronger than the gender divide.

\section{Method}

\subsection{Participants}

There is a general consensus in literature that SDO is a stable individual difference [51] although can relatively vary across some conditions. According to personenvironment fit theory, authors [27] posited that people select hierarchy-enhancing environments according to their SDO levels, as well as environments attract and socialize people according to how much in such places hierarchy-enhancing myths are supported. As a result, high-SDO people tend to fit better in hierarchy-enhancing environments and low-SDO people tend to fit better in hierarchy-attenuating environments (e.g., [18, 27, 52]). In the present chapter, we opted to study the gender invariance hypothesis in people belonging to political groups with a different support of hierarchy-enhancing legitimizing myths accordingly to the literature that outlines that the SDO level among participants (of both sex) of political groups mirrors the different extents to which parties support group dominance.

We included 626 subjects, 350 males and 276 females, who had been for at least 2 years activists in political parties or associations belonging to two well-differentiated groups of (1) extreme left-wing and (2) extreme right-wing and having also two additional groups of (3) center left-wing and (4) center right-wing parties. We decided to invest considerable effort to secure a relatively large sample of political activists of different political parties. We sent emails to the address of local political parties asking to give the questionnaire to the activists. The questionnaire was accompanied by a letter of the Sapienza University of Rome stationery presenting the aims of the scientific research and guaranteeing anonymity and privacy. After mailing questionnaires and letters, we tried to recontact the political parties to ascertain that they received the questionnaires and to solicit their responses. However, since the response rate was low, after the first contacts had been established, snowball sampling was used to recruit other political activists:

1. Extreme left activists were 126, 70 males and 56 females (mean age 26.21, SD 4.83; range 16-34). About 9\% have a low level of education, $61 \%$ a high school diploma, and $30 \%$ a college degree. These extreme left-wing organizations define themselves as supporting socialist, feminist, and ecologist issues and fighting against all social inequalities based on race, ethnicity, gender, or other discriminating features. Activists spend considerable time in these groups in weekly meetings and organizing protest marches, boycotts, and sit-in in favor of oppressed groups.

2. Extreme Right activists were 181, 123 males and 58 females (mean age 24.01, SD 5.07; range 14-34). About $23 \%$ had a college degree, $57 \%$ had a high school diploma, and about $20 \%$ did not finish high school. These groups promote attitudes and belief systems such as nationalism, racism, classism, sexism, ethnocentrism, and political-economic conservatism. Extreme right activists also meet weekly and often engage in nontraditional political activities bordering on illegality such as unauthorized protest marches that sometimes end in violence (fights with police officers or leftwing extremists). 
3. Center left-wing activists were 111, 50 males and 61 females (mean age 26.71, SD 4.86; range 16-35). They are members of the center left-wing moderate parties. About $7 \%$ have a junior high diploma, $67 \%$ a high school diploma, and $26 \%$ a college degree. They engage in more traditional legal-political activities such as signing petitions, political campaigns, raising funds, and getting people to the voting polls.

4. Center right-wing activists were 208, 107 males and 101 females (mean age 27.20, SD 4.76; range 14-35). They belong to center right-wing parties. About $68 \%$ have a high school diploma, $24 \%$ a college degree, and $8 \%$ a junior high education. They also engage in more traditional party activities, like organizing fundraising events, helping party candidates, distributing documents, and getting voters to the poll.

\subsection{Instruments}

All subjects filled a questionnaire which contained:

1. A section in which subjects recorded age, sex, and educational level. Furthermore, to confirm and control the distinctive SS belonging to the selected groups, we measured their political orientation by means of a single item (a 10-point scale), where point 1 meant extreme right-wing orientation and point 10 meant extreme left-wing orientation.

2. SDO scale used was an Italian adaptation of the SDO scale [53].

\section{Statistical analyses and results}

We first performed an analysis of variance to ascertain the political orientation as firmly acting differences within groups in SDO males and females scores.

Results are shown in Table 1.

To deepen the test of the invariance hypothesis, we applied a multiple regression model involving social dominance orientation and gender across the four groups considered. A multigroup path analysis was performed to test the hypothesis of the influences of gender on social dominance, this way independently of political orientation. A dummy variable was created with 1 corresponding to males. Thus, in our regression model, the weight is the average difference between males and females on SDO.

SDO was treated as an exogenous latent variable with three indices. The latter was formed summing up groups of items of the scale. The figure below illustrates the conceptual model (Figure 1).

Several competing models were tested.

Hypotheses were as follows in ascending order of constraints:

- H1: both factor loadings and regression weight are different for each of the four political orientation groups.

- HOA: regression weight is invariant for each of the political groups.

- HOB: factor loadings of social dominance are invariant for each of the four political groups.

- H0C: both regression weight and factor loading are invariant for each of the political groups. 
Results showed in Table 2 ascertain from these results that hypotheses H1 and H0A showed a relevant good fit.

In order to choose the best model, D2 statistic was calculated [54] as follows:

$$
\begin{gathered}
\mathrm{D} 2=\mathrm{Chi}{ }^{2} \mathrm{HOA}-\mathrm{Chi}{ }^{2} \mathrm{H} 1 \\
\mathrm{df}=\mathrm{dfHOA}-\mathrm{dfH} 1
\end{gathered}
$$

So,

$$
\begin{gathered}
\mathrm{D} 2=18.37-13.86 \\
\mathrm{df}=11-8=3 \\
\mathrm{p}=0.211 .
\end{gathered}
$$

\begin{tabular}{|c|c|c|c|c|c|c|c|c|}
\hline \multirow[t]{2}{*}{ Gender } & \multirow[t]{2}{*}{$\begin{array}{c}\text { Political } \\
\text { orientation }\end{array}$} & \multirow[t]{2}{*}{ Means } & \multicolumn{2}{|l|}{ Gender } & \multicolumn{2}{|c|}{$\begin{array}{c}\text { Political } \\
\text { orientation }\end{array}$} & \multicolumn{2}{|c|}{$\begin{array}{c}\text { Gender } \times \\
\text { political } \\
\text { orientation }\end{array}$} \\
\hline & & & $F$ & $P$ & $F$ & $p$ & $F$ & $p$ \\
\hline \multirow[t]{5}{*}{ Males } & $\begin{array}{l}\text { Extreme } \\
\text { right-wing }\end{array}$ & 3.74 & 19.85 & $<0.001$ & 192.00 & $<0.001$ & 3.16 & $<0.05$ \\
\hline & Center right & 3.27 & & & & & & \\
\hline & Center left & 1.84 & & & & & & \\
\hline & $\begin{array}{l}\text { Extreme } \\
\text { left-wing }\end{array}$ & 1.73 & & & & & & \\
\hline & Total & 2.93 & & & & & & \\
\hline \multirow[t]{5}{*}{ Females } & $\begin{array}{c}\text { Extreme } \\
\text { right-wing }\end{array}$ & 3.12 & & & & & & \\
\hline & Center right & 3.03 & & & & & & \\
\hline & Center left & 1.79 & & & & & & \\
\hline & $\begin{array}{l}\text { Extreme } \\
\text { left-wing }\end{array}$ & 1.46 & & & & & & \\
\hline & Total & 2.46 & & & & & & \\
\hline
\end{tabular}

Table 1.

Four political groups, male and female-SDO means.

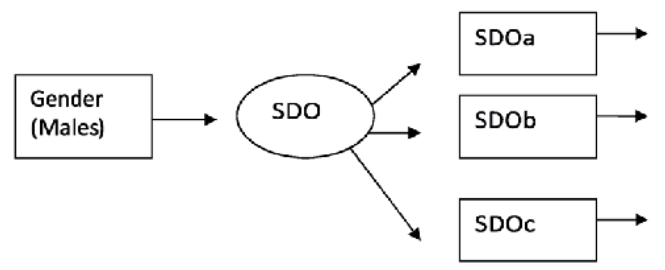

Figure 1.

Conceptual model of the regression of gender on social dominance orientation. 
Political Gender Gap and Social Dominance Orientation

DOI: http://dx.doi.org/10.5772/intechopen.92222

\begin{tabular}{lccc}
\hline Model & Chi & df & P \\
\hline H1 & 13.86 & 8 & 0.09 \\
\hline H0A & 18.37 & 11 & 0.07 \\
\hline H0B & 23.86 & 14 & 0.05 \\
\hline H0C & 29.76 & 17 & 0.03 \\
\hline
\end{tabular}

Table 2.

Results of path analysis of the model tested.

Since the reduction in $\mathrm{Chi}^{2}$ is not statistically significant, it is possible to choose the model of the invariance of regression weight among the political groups. Focusing on this model, fit indexes were RMSEA $=0.07$; NFI $=0.98$; and CFI $=0.99$.

Loading differences among the groups did not seem due to a different factorial structure.

Concerning the main hypothesis, these results showed that males express higher SDO than females invariantly of their right-wing or left-wing political orientation (beta $=0.21 ; \mathrm{R} 2=0.04)$.

\section{Discussion}

Recent years have seen a rise in the political gender gap; women in Western European countries have gradually preferred more leftist parties [55]. In the United States, the share of women who identify with or lean toward the Democratic Party has increased. The last data from the Pew Research Center [56] show that among registered voters, $56 \%$ of women affiliate or favor the Democratic Party compared to $44 \%$ of men. Several authors have hypothesized that the difference between women and men on political attitudes and political party identification can be attributed to differences in SDO [5]. To investigate if the gender gap in politics goes deeper than traditional left-wing and right-wing division, we analyzed gender differences in SDO in activists of HE and HA political parties. According to the gender invariance hypothesis, all else been equal, men should still have significantly higher SDO than women. The predisposition of males to be temperamentally inclined to dominate will produce nonetheless residual gender differences even among males and females belonging to groups who uphold egalitarian group values.

To analyze the gender invariance hypothesis in a political context, we based our study on political activists. Political activists, in fact, not only identify with groups that hold specific hierarchy-enhancing or hierarchy-attenuating ideologies but actively participate, promoting their values and ideas within the group. Their active commitment in specific HE or HA groups should encourage in fact, even more, the internalization of legitimizing myths that may influence their levels of SDO. Our research was based on four samples of male and female activists belonging to hierarchy enhancing - right-wing - vs. hierarchy attenuating - left-wing - political groups. According with SIT and SDT, the SDO scores should be higher in right-wing groups than left-wing groups and highest in extreme rightwing groups and lowest in extreme left groups. However, according to SDT, even while absolute levels of SDO may vary across situations, men should still have significantly higher SDO than women. The SIT theory, on the contrary, would predict that groups on both sides of the political divide should attract males and females who, for the left, are strong egalitarian and do not favor the oppression of one group over another and, for the right males and females, who hold equally strong opposite views. Gender differences should be insignificant since egalitarian adult socialization 
experiences should promote egalitarian attitudes in both men and women activists and vice versa.

On the whole, our results sustain more the validity of the soft than the strong version of the invariance hypothesis [25]. We observed general differences in SDO score across political groups (e.g., SDO score for right-wing were higher than leftwing); therefore, males of all the activists' groups showed a higher social dominance than females invariantly belonging to left- or right-wing political groups. The soft version implies indeed that SDO difference between men and women should be essentially constant across cultural and situational factors, everything else being equal. Our study confirmed that SDO differentiated men and women invariantly across cultural and situational factors such as political activism practiced both in moderate and extremist political groups. Male right-wing extremists had the highest SDO scores and female left-wing extremists the lowest. Still, the gender differences persisted in all groups, giving strong support for the temperamental differences in dominance predisposition.

Our data did show also that extreme right-wing women presented significantly higher scores of SDO than men belonging to left-wing parties. This result can be congruent with both SIT and SDT, which emphasizes the importance of group identification and the soft version of the SDO gender hypothesis that recognizes the influence of context.

\section{Conclusions}

In this study, the recent increase of the gender gap in political elections was addressed by analyzing gender differences in SDO of political activists. Our findings confirm the soft version of gender invariance hypothesis. However, our study has several limits; we still do not know if people who chose these different groups had originally higher or lower SDO or if the experience of belonging to different groups changes the SDO. Furthermore, we did not explore whether changes in SDO occurred in people who were active participants in different political groups. Future studies should ascertain whether SDO scores diminish after being an active member of the political moderate leftist group and if these changes occur in less time or more often when participating in extremist groups with even stronger egalitarian ideals. Moreover, to understand more the influence of group identification maintained by the SIT, the number of years of group involvement should be taken into consideration in future studies. Future research could also explore if new media like partisan Internet sites and social networks can enhance or reduce SDO.

Our results, with their present limits, also show that SDO is strongest in males and females in far right-wing groups, and these findings could help explain why these groups are now rising in recent elections in Europe and several other countries worldwide. One fundamental belief of SDT theory is that certain groups of people are entitled to rule over other groups [13]. The SDO scale elicits agreementdisagreement with statements such as "it's a good thing that some groups are at the top, and others at the bottom" or "Some groups of people are simply inferior to other groups." "America first," which Trump launched, had a very precise meaning, to favor American-born citizens over immigrants. This slogan has been echoed by right-wing nationalist parties in Italy ("Italy first") and Hungary, Poland, and Turkey. In all these countries, authoritarian leaders have emerged and are sustained by male and female supporters, who perceive themselves similar to their leaders, and in fact, both authoritarian leaders and followers probably share very high SDO. As a matter of fact, recent studies have shown that SDO is related to support for radical right parties [57]. 


\section{Author details}

Minou Ella Mebane ${ }^{1 *}$, Antonio Aiello ${ }^{2}$ and Donata Francescato ${ }^{3}$

1 University Giustino Fortunato, Benevento, Italy

2 University of Pisa, Pisa, Italy

3 ASPIC (School of Specialization in Community Clinical Psychology and Integrated Humanistic Psychotherapy), Rome, Italy

*Address all correspondence to: me.mebane@unifortunato.eu

\section{IntechOpen}

(C) 2020 The Author(s). Licensee IntechOpen. This chapter is distributed under the terms of the Creative Commons Attribution License (http://creativecommons.org/licenses/ by/3.0), which permits unrestricted use, distribution, and reproduction in any medium, provided the original work is properly cited. (cc) BY 


\section{References}

[1] Center for the American Woman and Politics. The Gender Gap Voting Choices in Presidential Elections. 2017. Available from: http://www.cawp.rutgers.edu [Accessed: 03 January 2020]

[2] Eagly AH, Diekman AB. Examining gender gaps in sociopolitical attitudes: It's not Mars and Venus. Feminism and Psychology. 2006;16(1):26-34. DOI: 10.1177/0959-353506060817

[3] Shorrocks R. Cohort change in political gender gaps in Europe and Canada: The role of modernization. Politics and Society. 2018;46(2):135-175. DOI: $10.1177 / 0032329217751688$

[4] Conover PJ, Sapiro V. Gender, feminist consciousness, and war. American Journal of Political Science. 1993;37(4):1079-1099

[5] Pratto F, Sttallworth LM, Sidanius J. The gender gap: Differences in political attitudes and social dominance orientation. The British Journal of Social Psychology. 1997;36:49-68

[6] Sidanius J, Liu JH. The Gulf war and the Rodney king beating: Implications of the general conservatism and social dominance perspectives. The Journal of Social Psychology. 1992;132(6):685-700

[7] Eagly AH, Diekman AB, Johannesen-Schmidt MC, Koenig AG. Gender gaps in sociopolitical attitudes: A social psychological analysis. Journal of Personality and Social Psychology. 2004;87(6):796-816

[8] Ekehammar B, Nilsson I, Sidanius J. Social attitudes and social status: A multivariate and multinational analysis. Personality and Individual Differences. 1987;10:203-208. DOI: 10.1016/0191-8869(89)90205-5
[9] Altemeyer B. Right-Wing

Authoritarianism. Manitoba: University of Manitoba Press; 1981

[10] Altemeyer B. The Authoritarian Specter. Cambridge, MA: Harvard University Press; 1996

[11] Ekehammar B. Sex differences in socio-political attitudes revisited. Educational Studies. 1985;11(1):3-9

[12] Boehnke K, Schwartz S. Fear of war: Relations to values, gender, and mental health in Germany and Israel. Peace and Conflict. 1997;3:149-165. DOI: 10.1207/ s15327949pac0302_3

[13] Sidanius J, Pratto F. Social Dominance: An Intergroup Theory of Social Hierarchy and Oppression. Cambridge, MA: Cambridge University Press; 1999

[14] Levin S. Perceived group status differences and the effects of gender, ethnicity, and religion on social dominance orientation. Political Psychology. 2004;25(1):31-48

[15] Sidanius J, Levin S, Liu J, Pratto F. Social dominance orientation, anti-egalitarianism, and the political psychology of gender: An extension and cross-cultural replication. European Journal of Social Psychology. 2000;30(1):41-67

[16] Ho AK, Sidanius J, Kteily N, Sheehy-Skeffington J, Pratto F, Henkel KE, et al. The nature of social dominance orientation: Theorizing and measuring preferences for intergroup inequality using the new SDO7 scale. Journal of Personality and Social Psychology. 2015;109:1003-1028. DOI: 10.1037/pspi0000033

[17] Pratto F, Sidanius J, Stallworth LL, Malle FB. Social dominance orientation: A personality variable predicting 
social and political attitudes. Journal of Personality and Social Psychology. 1994;67(4):741-763

[18] Pratto F, Sidanius J, Levin S. Social dominance theory and the dynamics of intergroup relations: Taking stock and looking forward. European Review of Social Psychology. 2006;17:271-320. DOI: $10.1080 / 10463280601055772$

[19] Aiello A, Passini S, Tesi A, Morselli D, Pratto F. Measuring support for intergroup hierarchies: Assessing the psychometric proprieties of the social dominance Orientation7 scale. Testing, Psychometrics, Methodology in Applied Psychology. 2019;26(3):373-383. DOI: 10.4473/TPM26.3.4

[20] Aiello A, Tesi A, Pierro A, Pratto F. Social dominance and interpersonal power: Asymmetrical relationships within hierarchyenhancing and hierarchy-attenuating work environments. Journal of Applied Social Psychology. 2018;48(1):35-45. DOI: $10.1111 /$ jasp. 12488

[21] Tesi A, Aiello A, Pratto F, Pierro A. The spiral of oppression in work organizations: Framing social dominance theory and the interpersonal power interaction model. Testing, Psychometrics, Methodology in Applied Psychology. 2019;26(3):363-372. DOI: 10.4473/TPM26.3.3

[22] Tesi A, Aiello A, Morselli D, Giannetti E, Pierro A, Pratto F. Which people are willing to maintain their subordinated position? Social dominance orientation as antecedent to compliance to harsh power tactics in a higher education setting. Personality and Individual Differences. 2019;151. DOI: 10.1016/j.paid.2019.04.045

[23] Pratto F, Walker A. The bases of gendered power. In: Eagly AH, Beall AE, Sternberg RJ, editors. The Psychology of Gender. New York, NY: Guilford Press; 2004. pp. 242-268
[24] Pratto F, Stallworth LM, Sidanius J, Siers B. The gender gap in occupational role attainment: A social dominance approach. Journal of Personality and Social Psychology. 1997;72:37-53. DOI: 10.1037/0022-3514.72.1.37

[25] Sidanius J, Pratto F, Bobo L. Social dominance orientation and the political psychology of gender: A case of invariance? Journal of Personality and Social Psychology. 1994;67:998-1011

[26] Zakrisson I. Gender differences in social dominance orientation: Gender invariance may Be situation invariance. Sex Roles. 2004;59:254-263

[27] Haley H, Sidanius J. Personorganization congruence and the maintenance of group-based social hierarchy: A social dominance perspective. Group Processes \& Intergroup Relations. 2005;8:187-203.

DOI: $10.1177 / 1368430205051067$

[28] Sidanius J, Pratto F. Social dominance theory and the dynamics of inequality: A reply to Schmitt, Branscombe, \& Kappen and Wilson \& Liu. The British Journal of Social Psychology. 2003;42:207-213

[29] Heaven PCL, Bucci S. Right-wing authoritarianism, social dominance orientation and personality: An analysis using the IPIP measure. European Journal of Personality. 2001;15:49-56

[30] Sidanius J, Sinclair S, Pratto F. Social dominance orientation, gender, and increasing educational exposure. Journal of Applied Social Psychology. 2006;36(7):1640-1653

[31] Wilson MS, White JT. Assessing the 'Gender Gap' in New Zealand politics: The mediating effects of social dominance orientation in student and general population. New Zealand Journal of Psychology. 2010;39(1):38-44 
[32] Sidanius J, Pratto F, Brief D. Group dominance and the political

psychology of gender: A cross- cultural comparison. Political Psychology. 1995;16:381-396.10.2307/3791836

[33] Lee IC, Pratto F, Johnson TB. Intergroup consensus/disagreement in support of group based hierarchy: An examination of socio-structural and psycho- cultural factors. Psychological Bulletin. 2011;137(6):1029-1064. DOI: 10.1037/a0025410

[34] Bates C, Heaven PCL. Attitudes to women in society: The role of social dominance orientation and social values. Journal of Community and Applied Social Psychology. 2001;11: 43-49. DOI: 10.1002/ casp.589

[35] Lehmiller JJ, Schmitt MT. Intergroup attitudes and values in response to the U.S. invasion of Iraq. Peace and Conflict: Journal of Peace Psychology. 2008;14:259-274. DOI: 10.1080/10781910802229231

[36] McIntyre $\mathrm{MH}$, Barrett ES, McDermott R, Johnson DDP, Cowden J, Rosen SP. Finger length ratio (2D: 4D) and sex differences in aggression during a simulated war game. Personality and Individual Differences. 2007;42:755764. DOI: 10.1016/j.paid.2006.08.009

[37] Morrison TG, Kenny P, Harrington A. Modern prejudice toward gay men and lesbian women: Assessing the viability of a measure of modern homonegative attitudes within an Irish context. Genetic, Social, and General Psychology Monographs. 2005;13(3):219-250

[38] Snellman A, Ekehammar B. Ethnic hierarchies, ethnic prejudice, and social dominance orientation. Journal of Community and Applied Social Psychology. 2005;31(3):369-409

[39] Pratto F, Liu JH, Levin S, Sidanius J, Shih M, Bachrach H, et al.
Social dominance orientation and the legitimization of inequality across cultures. Journal of Cross-Cultural Psychology. 2000;31:369-409

[40] Küpper B, Zick A. Inverse gender gap in Germany: Social dominance orientation among men and women. Journal international de psychologie. 2011;46:33-45. DOI: 10.1080/00207594.2010.491121

[41] Wilson SM, Liu HG. Social dominance orientation and gender: the moderating role of gender. British Journal of Social Psychology. 2003;42:187-198

[42] Huanga L, Liu J. Personality and social structural implications of the situational priming of social dominance orientation. Personality and Individual Differences. 2005;38(2):267-276

[43] Foels R, Pappas CJ. Learning and unlearning the myths we are thought: Gender and social dominance orientation. Sex Roles. 2004;50:743-757

[44] Kemmelmeier M, Danielson C, Basten J. What's in a grade? Academic success and political orientation. Personality and Social Psychology Bulletin. 2005;1(10):1386-1399

[45] Dambrun M, Guimond S, Duarte S. The impact of hierarchyenhancing vs. attenuating academic major on stereotyping: The mediating role of perceived social norm. Current Research in Social Psychology. 2002;7:114-136

[46] Batalha L, Reynolds KJ, Newbigin CA. All else being equal: Are men always higher in social dominance orientation than women. European Journal of Social Psychology. 2011;41:796-806

[47] Seyle DC, Newman ML. A house divided? The psychology of red and blue 
America. The American Psychologist. 2006;61:571-580

[48] McCloskey H, Hoffman P, O'Hara R. Issue conflict and consensus among party leaders and followers.

The American Political Science Review. 1960;56:406-429

\section{[49] Huddy L. Contrasting} theoretical approaches to intergroup relations. Political Psychology. 2004;25(6):947-967

[50] Hyde JS. The gender similarities hypothesis. The American Psychologist. 2005;60:581-592. DOI: 10.1037/0003-066X.60.6.581

[51] Kteily NS, Sidanius J, Levin S. Social dominance orientation: Cause or'mere effect'? Evidence for SDO as a causal predictor of prejudice and discrimination against ethnic and racial outgroups. Journal of Experimental Social Psychology. 2011;47:208-214. DOI: 10.1016/j.jesp.2010.09.009

[52] Tesi A, Pratto F, Pierro A, Aiello A. Group dominance in hierarchyattenuating and hierarchy-enhancing organizations: The role of social dominance orientation, need for cognitive closure, and power tactics in a person-environment (Mis) fit perspective. Group Dynamics: Theory, Research, and Practice. 2020. DOI: 10.1037/gdn0000117 (Advance online publication)

[53] Di Stefano G, Roccato M. Una banca di item per misurare l'orientamento alla dominanza sociale in Italia. [An item bank to measure the orientation towards social dominance in Italy]. TPM. 2005;12:5-20

[54] Jöreskog K, Sorbom D. LISREL 8: Structural Equation Modelling with the SIMPLIS Command Language. Chicago: Scientific Software International Inc; 1993
[55] Abendschön S, Steinmetz S. The gender gap in voting revisited: Women's party preferences in a European context. Social Politics. 2014;21(2):315-344. DOI: 10.1093/sp/jxu009

[56] Pew Research Center. Trends in party affiliation among demographic groups. 2018. Available from: https:// www.pewresearch.org

[57] Aichholzera J, Zandonella M. Psychological bases of support for radical right parties. Personality and Individual Differences. 2016;96:185-190 



\title{
Psychological Health Influences of Legal-Marriage and -Partnerships on Same-Sex Couples
}

\author{
William N. Elwood, Veronica L. Irvin, Benmei Liu, \\ Richard Lee and Nancy Breen
}

\begin{abstract}
This chapter explores whether Californians in same-sex legal marriages and partnerships reported lower levels of psychological distress than other adult Californians after the 2008 California Supreme Court Decision that legalized samesex marriage. We pooled 10 years of California Health Interview Survey (CHIS) data and employ a T1-T2 design to approximate a time series design. Dependent variables include overall self-related health, psychological distress, and household income. Independent variables include sexual identity and same-sex spouse.

Bi-variate analyses compared self-reported mental and physical health between the two periods. We found decreased reports of poorer health and increased reports of very good health among gay men and lesbian women with legal spouses. Psychological distress decreased for legally coupled gay men and lesbians while increased slightly among unpartnered lesbian women and gay men. Household income increased among coupled lesbian women and gay men and decreased among others. Our project demonstrated positive health influences for Californians with legal same-sex spouses. We recommend future research projects that explore whether and how same- and opposite-sex marriage benefits health, well-being, and prosperity, and for marital status survey questions that are inclusive of sexual and gender identities and elicit the sex/gender of a respondent's spouse.
\end{abstract}

Keywords: same-sex marriage and partnerships, mental health, survey measurement

\section{Introduction}

The U.S. federal government continues to increase the collection of sexualorientation data in its surveys [1-4], recognizes the need to develop a model for LGBT health that integrates behavioral, environmental, and socioeconomic factors, and intends to develop a framework, "to improve the health and well-being of people, ... enhancing prosperity in the community and for its residents and businesses" [5] Marriage is one social contract long associated with health, longevity, and prosperity for people in such relationships [6-10]. During the period that preceded local, state, and then national legalization of same-sex marriage in the U.S. [11], researchers analyzed secondary data and proposed that such legal recognition could ensure some of the health and financial benefits that opposite-sex married couples have long-enjoyed $[9,12,13]$. 
Research on effects of state-based same-sex marriage or legal-partnerships found more nuanced results [14-16], for example, increased health insurance coverage among California legally-partnered lesbians compared to heterosexual women with no change among gay men compared to heterosexual men [17]. An Illinoisbased study found similar findings among sexual minority women with even more profound effects of insurance coverage among racial/ethnic sexual minority women [18]. Another study found higher odds among legally-partnered California gay men to cite continuous health insurance coverage and regular medical providers than married heterosexual men-yet self-reported poorer health and well-being than heterosexual counterparts [19].

Since 2008, the California Health Interview Survey (CHIS) asked questions on same-sex marriage and legal partnerships [20] to reflect the state's policy progression from requiring employer-sponsored health insurance to same-sex partners in 2005, to locality-based same-sex marriage, to a 2008 State Supreme Court decision that affirmed same-sex marriage. Litigation regarding a statewide ballot initiative led to a suspension of issuing same-sex marriage licenses until 2013 when same-sex marriage licenses were issued once again.

The aim of this article is to explore whether Californians in same-sex legal partnerships and marriages reported lower levels of psychological distress after the 2008 California Supreme Court Decision that legalized same-sex marriage. The stress of homoprejudicial experiences has cumulative negative influences on the actual and perceived mental and physical health among lesbian and gay people [21]. Moreover, there is literature that demonstrates reciprocal links between psychosocial stress, health, and well-being [22, 23].

\section{Methods}

Our sample includes adult Californians surveyed before and after the California Supreme Court decision (CSCD) in 2008. We obtained data from CHIS for years 2005-2015. Initially fielded biennially, CHIS became a continuous survey in 2011. Administered in five languages, it employs a multi-stage probability design that selects subjects by random-digit dial within geographic strata. Respondents in this analysis include adults ages 18-70 who self-identified as heterosexual, lesbian/gay female, or gay male. CHIS did not ask sexual-identity questions of participants older than 70 [24]. We excluded respondents who said they were bisexual, celibate, non-sexual, or provided no response because the CHIS survey did not ask the sex/gender of a respondent's spouse [19] and thus lacked the ability to safely intuit the sex/gender of each respondent's spouse. CHIS obtained human subject approval for participant recruitment and data collection through the University of California, Los Angeles (UCLA). The National Institutes of Health's Office of Human Subjects Research Protection determined our study to be exempt from review as it involved the study of existing data recorded such that subjects cannot be identified.

\subsection{Dependent variable}

\subsubsection{Psychological distress}

CHIS has fielded the Kessler 6-item (K6) scale to assess nonspecific psychological distress since 2005 [25]. The K6 measures symptoms during the past 30 days: felt nervous, hopeless, restless or fidgety, worthless, depressed, and felt that everything 
was an effort-using Likert scales from 0 representing none of the time, to 4 representing all the time. The $\mathrm{K} 6$ scale is summed with scores of 0 representing lowest, and 24 representing the highest psychological distress level. Dichotomized moderate mental distress is defined as the sum of $\mathrm{K} 6$ scores at or above 5, the optimal lower threshold indicative of moderate mental distress [13]. The K6 continuous measure and the dichotomized moderate mental distress scores have demonstrated reliability and validity in population datasets, including CHIS [26, 27]. We used the dichotomous measure given our small subsample of married and partnered same-sex couples.

\subsection{Independent variables}

\subsubsection{Sexual identity}

CHIS participants self-reported as, "straight or heterosexual, as gay, lesbian or homosexual, bisexual, or other."

\subsubsection{Legal marriage and partnership}

CHIS asked all participants the standard marital question, "Are you now married, living with a partner in a marriage-like relationship, widowed, divorced, separated, or never married?" The response options do not include same-sex marriage or legal partnership. Previous research has shown that lesbian/gay women and gay men under-report marriage and legal partnership when responding to standard marital status questions [19]. To address under-report of marriage and to reflect the 2008 CSCD, CHIS asked all participants who reported having sex with someone of the same sex within the preceding 12 months whether that sexually-active respondent had a legal same-sex spouse or domestic partner. In addition to legallypartnered and married, we consolidated remaining status into other, a category including unmarried people who may be divorced, widowed, never-married, or living with a partner without legal recognition. We also constructed a binary variable for married/legally-partnered to increase the power of the data to find statistically significant results. There is no way to separate married and legally-partnered for data prior to 2009 because of questionnaire wording. In 2009, revised question wording distinguished married from legally-partnered. We compared percent of moderate psychological distress between married and legally-partnered lesbian women and gay men and found no statistical difference. Therefore, we collapsed married/legally-partnered as one group for our analyses in order to compare pre to post CSCD.

\subsubsection{Statistical analyses}

We performed all statistical analyses using CHIS data pooled from survey years 2005-2015 and weighted to the California population. Lesbian/gay women and gay men were compared to their heterosexual counterparts on sociodemographic variables. Data collected before 2008 were considered prior to the CSCD and data collected in 2008 and later were considered after the legal decision. The proportion of the sample experiencing moderate mental distress was plotted over time by gender, sexual identity, and couple status. Joinpoint analysis tested if trends in moderate psychological distress changed at specific years. Joinpoint uses weighted least squares to fit the trend model, using the inverse of the standard error as the weight variable. 
Bi-variate and logistic regression analyses compared psychological distress using the $\mathrm{K} 6$ scale between the periods before and after the CSCD. Bi-variate analyses were replicated only for lesbian/gay women and gay men and compared stress levels between those legally married or partnered as compared with those not. Independent variables of the logistic regression included the main effects of sexual identity, marriage/legal partnership and the timing of CSCD (before or after) and all the two-way interaction effects (i.e., sexual identity $x$ marriage/legal partnership, sexual identity $x$ timing of CSCD, marriage/legal partnership $x$ timing of CSCD) and the three-way interaction effect of the three variables (i.e., sexual identity $\mathrm{x}$ marriage/legal partnership $\mathrm{x}$ timing of CSCD) while adjusting for the following sociodemographics: Race, marital/partnered status, children in home, education, work status, income, geography, age. The conditional adjusted odds ratios compare the odds of reporting moderate mental health distress for that specific group pre- versus after-CSCD while holding all other variables constant.

\section{Results}

A total of 192,460 individuals were included in the analysis, with 6995 participants identifying as lesbian/gay women and gay men. Table 1 displays sociodemographics of lesbian, gay, and heterosexual individuals before and after the CSCD. There is an overall increase in non-white populations regardless of sexual orientation and a notable increase in reports of legal married/partnered lesbian women and gay men. Before 2008, only 11 percent of lesbian/gay female and gay male Californians reported being legally married/partnered. That percentage rose to 26.5\% after the state Supreme Court decision. Among heterosexuals, marriage slightly declined, from $57.2 \%$ before the ruling versus $51.8 \%$ after the decision. Table 1 also reports the mean distress score for sexual identity group pre versus post CSCD and shows that lesbian women and gay men reported higher scores of moderate distress than their heterosexual counterparts.

Table 2 provides descriptive results expressly for lesbian women and gay men by marital/partnered status pre versus post-CSCD. Employment was stable among married/partnered respondents, but unemployment increased among others.

Household income increased among married/partnered respondents but decreased among others. Notably, the percentage of respondents who had a child in the home decreased from 51 to $36 \%$ among married/partnered lesbian and gay respondents. Mean scores of moderate mental distress decreased for married or legally partnered but increased for other following the CSCD.

We explored the relationship the 2008 CSCD on self-reported moderate psychological distress among legally married or partnered and other respondents by sexual identity. A higher percentage of Lesbian women and gay men reported rates of distress than heterosexual counterparts. There was no change in the proportion of lesbian women and gay men who experienced moderate mental psychological distress before and after the CSCD. However, this result changes when we compare those who are legally partnered or married compared to other relationship status Legally married or partnered lesbian women and gay men were half as likely to report moderate psychosocial distress after the CSCD [OR, 0.52] as compared to prior to the CSCD. In contrast, moderate psychological distress remained relatively unchanged among other lesbian women and gay men [1.04], married heterosexuals [0.94] and other heterosexuals [0.94]. These results and their 95\% confidence intervals (CIs) for statistically significant relationships appear in Table 3.

We conducted Joinpoint analyses to determine if moderate psychological distress decreased at specific years. Figure 1 displays percent of respondents 


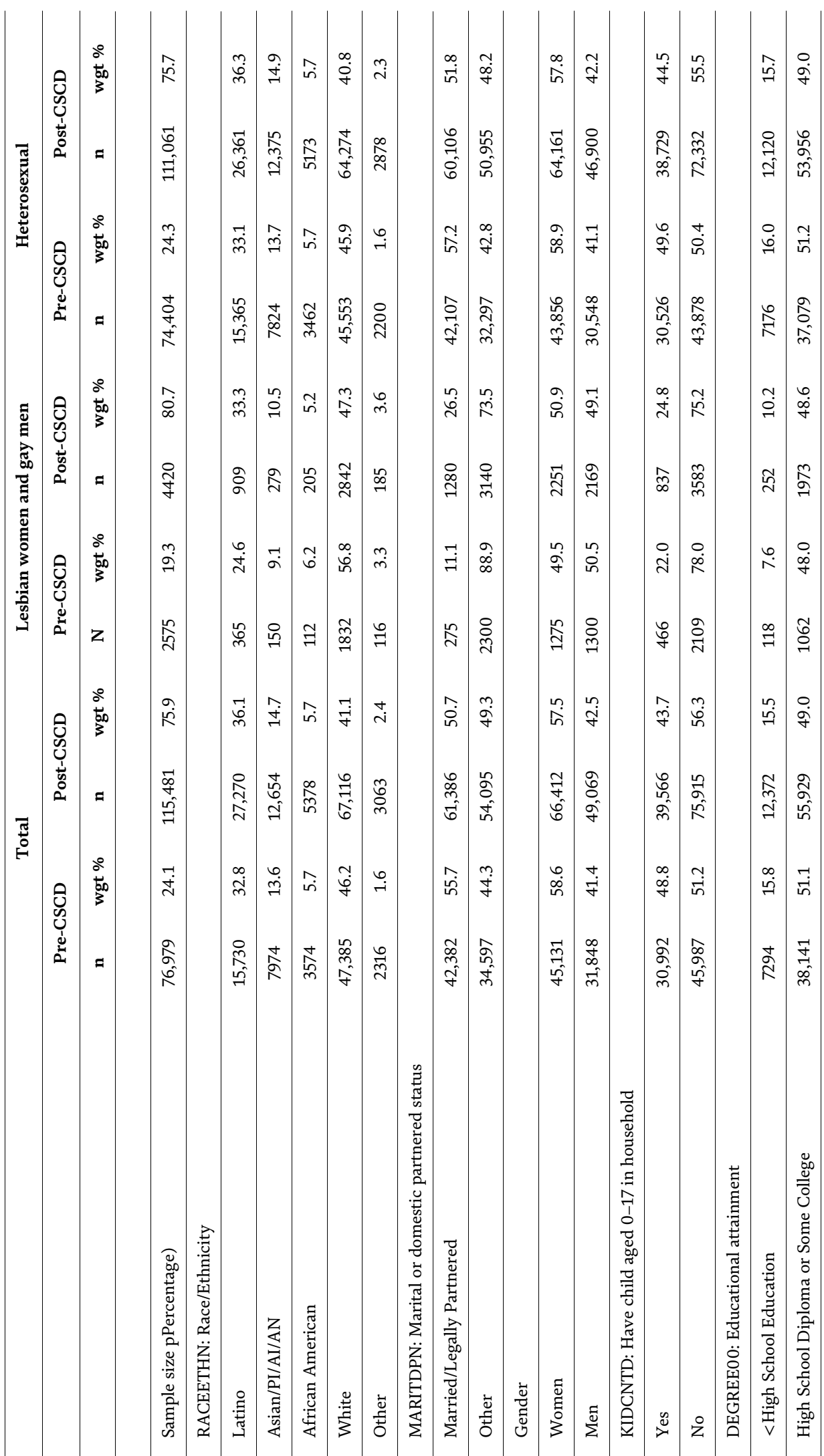




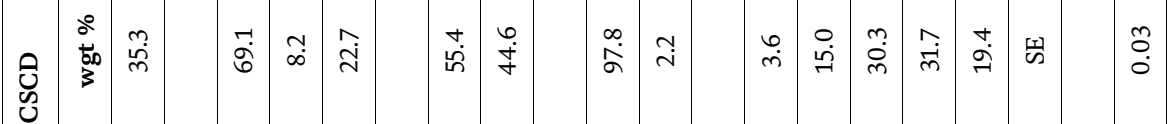

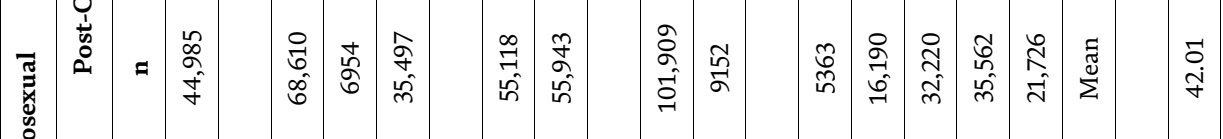

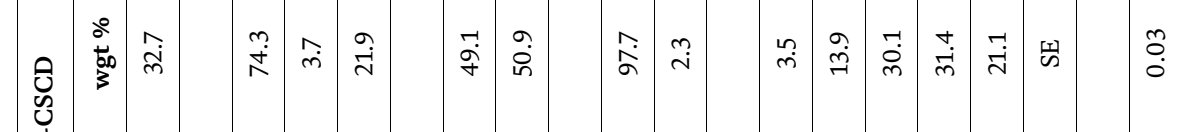

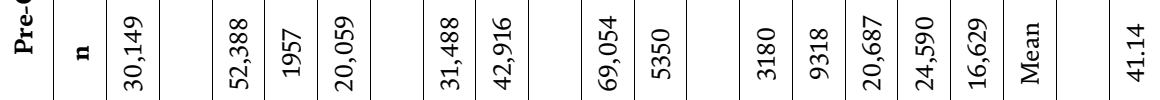

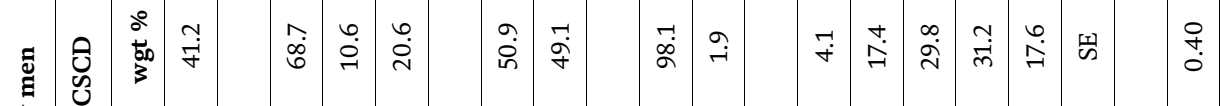

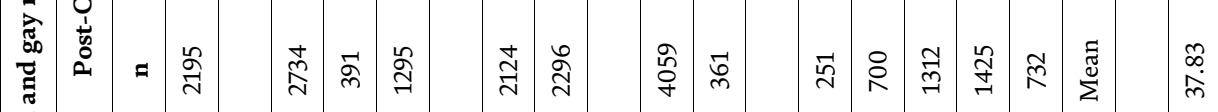

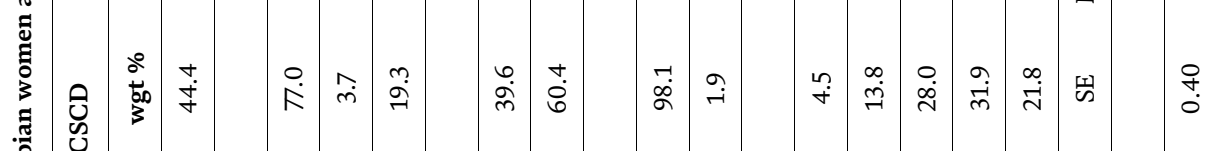

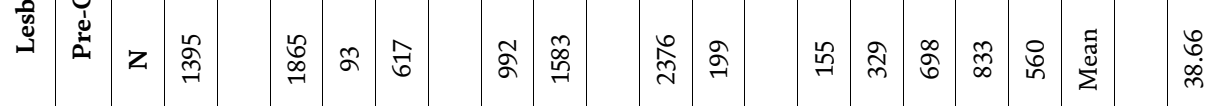

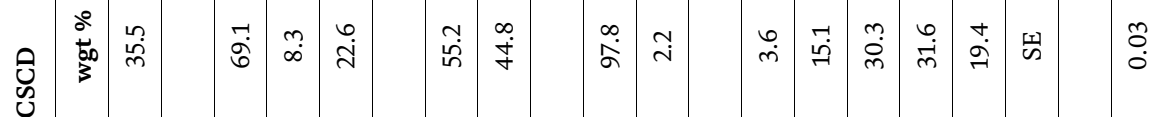

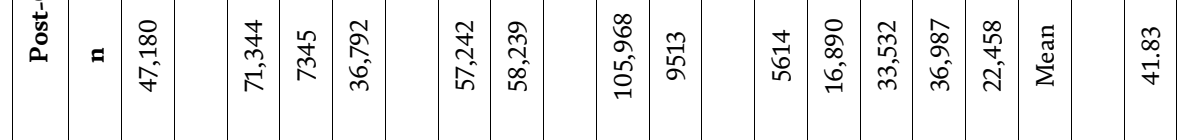
胥

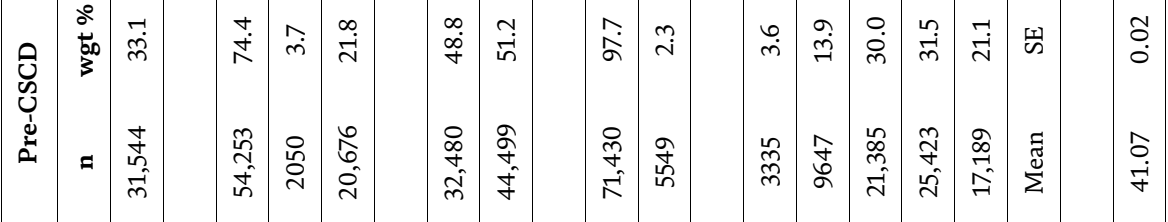

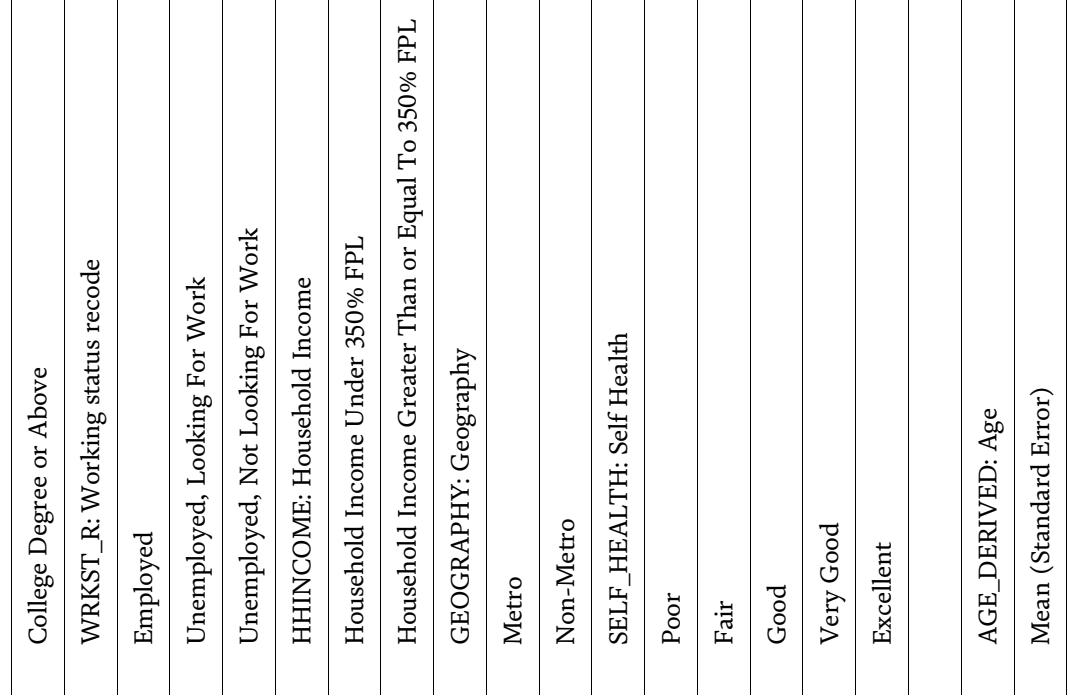


Psychological Health Influences of Legal-Marriage and-Partnerships on Same-Sex Couples DOI: http://dx.doi.org/10.5772/intechopen.90249

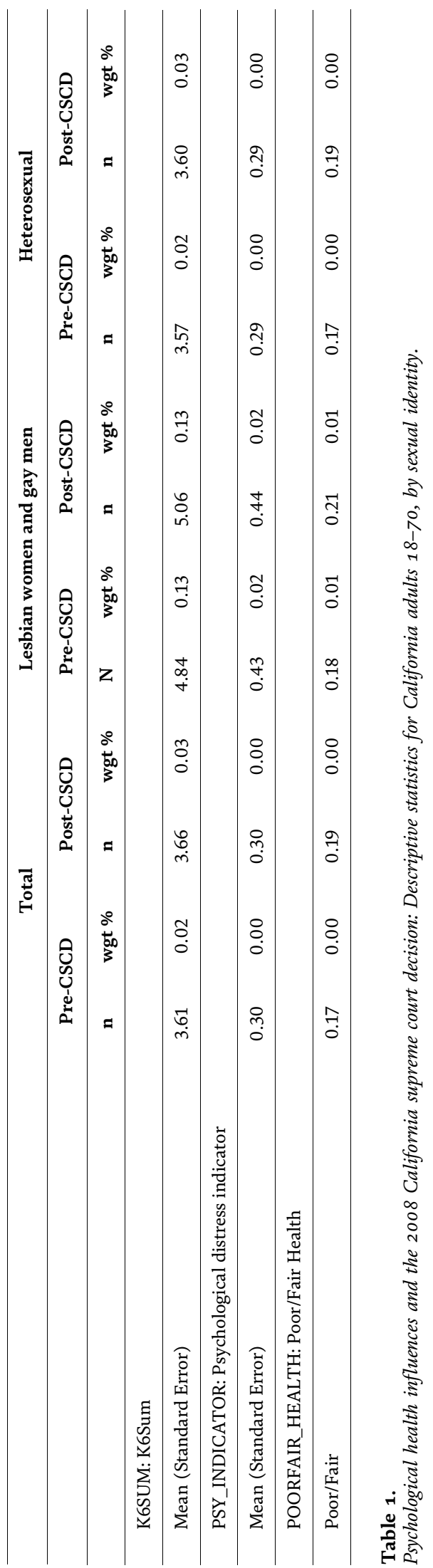




\begin{tabular}{|c|c|c|c|c|c|c|c|c|}
\hline & \multicolumn{4}{|c|}{ Married/legally partnered } & \multicolumn{4}{|c|}{ Other } \\
\hline & \multicolumn{2}{|c|}{ Pre-CSCD } & \multicolumn{2}{|c|}{ Post-CSCD } & \multicolumn{2}{|c|}{ Pre-CSCD } & \multicolumn{2}{|c|}{ Post-CSCD } \\
\hline & $\mathbf{n}$ & $\begin{array}{l}\text { wgt } \\
\%\end{array}$ & $\mathbf{N}$ & $\begin{array}{l}\text { wgt } \\
\%\end{array}$ & $\mathbf{n}$ & $\begin{array}{l}\text { wgt } \\
\%\end{array}$ & $\mathbf{N}$ & $\begin{array}{l}\text { wgt } \\
\%\end{array}$ \\
\hline Sample size (percentage) & 275 & 9.1 & 1280 & 90.9 & 2300 & 22.5 & 3140 & 77.5 \\
\hline \multicolumn{9}{|l|}{ Gender } \\
\hline Women & 184 & 66.9 & 763 & 59.6 & 1091 & 47.4 & 1488 & 47.4 \\
\hline Men & 91 & 33.1 & 517 & 40.4 & 1209 & 52.6 & 1652 & 52.3 \\
\hline \multicolumn{9}{|l|}{ RACEETHN: Race/ethnicity } \\
\hline Latino & 54 & 31.1 & 215 & 27.7 & 311 & 23.7 & 694 & 35.3 \\
\hline Asian/PI/AI/AN & 38 & 15.4 & 84 & 8.9 & 112 & 8.4 & 195 & 11.1 \\
\hline African American & 4 & 0.6 & 29 & 2.6 & 108 & 6.9 & 176 & 6.2 \\
\hline White & 169 & 50.8 & 908 & 57.7 & 1663 & 57.5 & 1934 & 43.6 \\
\hline Other & 10 & 2.1 & 44 & 3.1 & 106 & 3.5 & 141 & 3.9 \\
\hline \multicolumn{9}{|l|}{ KIDCNTD: Have child aged 0-17 in household } \\
\hline Yes & 135 & 50.9 & 362 & 35.6 & 331 & 18.4 & 475 & 20.9 \\
\hline No & 140 & 49.1 & 918 & 64.4 & 1969 & 81.6 & 2665 & 79.1 \\
\hline \multicolumn{9}{|l|}{ DEGREE: Educational attainment } \\
\hline$<$ High school education & 16 & 9.5 & 62 & 7.1 & 102 & 7.4 & 190 & 11.3 \\
\hline High school diploma or Some college & 117 & 42.8 & 455 & 39.7 & 945 & 48.6 & 1518 & 51.8 \\
\hline College degree or above & 142 & 47.7 & 763 & 53.2 & 1253 & 44.0 & 1432 & 36.9 \\
\hline \multicolumn{9}{|l|}{ WRKST_R: Working status recode } \\
\hline Employed & 194 & 72.6 & 858 & 72.3 & 1671 & 77.5 & 1876 & 67.5 \\
\hline Unemployed, looking for work & 9 & 4.1 & 58 & 5.1 & 84 & 3.6 & 333 & 12.6 \\
\hline Unemployed, not looking for work & 72 & 23.3 & 364 & 22.6 & 545 & 18.8 & 931 & 19.9 \\
\hline \multicolumn{9}{|l|}{ HHINCOME: Household Income } \\
\hline Household income under $350 \%$ FPL & 113 & 44.3 & 390 & 32.8 & 879 & 39.1 & 1734 & 57.4 \\
\hline $\begin{array}{l}\text { Household income greater than or equal to } \\
350 \% \text { FPL }\end{array}$ & 162 & 55.7 & 890 & 67.2 & 1421 & 60.9 & 1406 & 42.6 \\
\hline \multicolumn{9}{|l|}{ GEOGRAPHY: Geography } \\
\hline Metro & 250 & 97.4 & 1185 & 98.4 & 2126 & 98.2 & 2874 & 98.0 \\
\hline Non-metro & 25 & 2.6 & 95 & 1.6 & 174 & 1.8 & 266 & 2.0 \\
\hline \multicolumn{9}{|l|}{ SELF_HEALTH: Self Health } \\
\hline Poor & 11 & 4.4 & 54 & 3.0 & 144 & 4.5 & 197 & 4.4 \\
\hline Fair & 36 & 15.5 & 183 & 14.1 & 293 & 13.6 & 517 & 18.6 \\
\hline Good & 85 & 32.3 & 349 & 28.6 & 613 & 27.5 & 963 & 30.2 \\
\hline Very Good & 89 & 27.8 & 445 & 36.5 & 744 & 32.4 & 980 & 29.2 \\
\hline \multirow[t]{2}{*}{ Excellent } & 54 & 20.1 & 249 & 17.8 & 506 & 22.1 & 483 & 17.6 \\
\hline & Mean & $\mathrm{SE}$ & Mean & SE & Mean & $\mathrm{SE}$ & Mean & SE \\
\hline \multicolumn{9}{|l|}{ AGE_DERIVED: Age } \\
\hline Mean (standard error) & 40.64 & 1.04 & 44.01 & 0.65 & 38.41 & 0.42 & 35.61 & 0.43 \\
\hline
\end{tabular}


Psychological Health Influences of Legal-Marriage and-Partnerships on Same-Sex Couples DOI: $h$ ttp://dx.doi.org/10.5772/intechopen.90249

\begin{tabular}{|c|c|c|c|c|c|c|c|c|}
\hline & \multicolumn{4}{|c|}{ Married/legally partnered } & \multicolumn{4}{|c|}{ Other } \\
\hline & \multicolumn{2}{|c|}{ Pre-CSCD } & \multicolumn{2}{|c|}{ Post-CSCD } & \multicolumn{2}{|c|}{ Pre-CSCD } & \multicolumn{2}{|c|}{ Post-CSCD } \\
\hline & $\mathbf{n}$ & wgt & $\mathbf{N}$ & wgt & $\mathbf{n}$ & wgt & $\mathbf{N}$ & wgt \\
\hline & & $\%$ & & $\%$ & & $\%$ & & $\%$ \\
\hline \multicolumn{9}{|l|}{ K6SUM: K6Sum } \\
\hline Mean (standard error) & 5.09 & 0.38 & 4.14 & 0.22 & 4.81 & 0.14 & 5.40 & 0.17 \\
\hline \multicolumn{9}{|c|}{ PSY_INDICATOR: Psychological distress indicator } \\
\hline Mean (standard error) & 0.50 & 0.05 & 0.33 & 0.03 & 0.43 & 0.02 & 0.48 & 0.02 \\
\hline \multicolumn{9}{|c|}{ POORFAIR_HEALTH: Poor/Fair Health } \\
\hline Poor/Fair & 0.20 & 0.04 & 0.17 & 0.02 & 0.18 & 0.01 & 0.23 & 0.02 \\
\hline
\end{tabular}

Table 2.

Descriptive statistics for lesbian women and gay men by marital/partnered status.

Full sample of adults to compare lesbian/gay female, gay male, and heterosexual married/legallypartnered and otherwise single

\begin{tabular}{|c|c|c|c|c|}
\hline & \multicolumn{2}{|c|}{$\begin{array}{l}\text { All Lesbian and Gay } \\
\text { individuals }\end{array}$} & \multicolumn{2}{|c|}{$\begin{array}{l}\text { All Heterosexual } \\
\text { individuals }\end{array}$} \\
\hline & $\begin{array}{l}\text { Pre- } \\
\text { CSCD }\end{array}$ & $\begin{array}{l}\text { Post- } \\
\text { CSCD }\end{array}$ & $\begin{array}{l}\text { Pre- } \\
\text { CSCD }\end{array}$ & $\begin{array}{l}\text { Post- } \\
\text { CSCD }\end{array}$ \\
\hline Number of respondents & 2575 & 4420 & 74,404 & 111,061 \\
\hline Percent married or legally partnered & $11.1 \%$ & $26.5 \%$ & $57.2 \%$ & $51.8 \%$ \\
\hline $\begin{array}{l}\text { Psychological Distress mean and standard deviation } \\
\text { Kessler 6-item (K6) continuous score (0-24) }\end{array}$ & $\begin{array}{c}4.84 \\
(0.13)\end{array}$ & $5.06(0.13)$ & $\begin{array}{c}3.57 \\
(0.02)\end{array}$ & $3.60(0.03)$ \\
\hline $\begin{array}{l}\text { Percent reporting at least moderate mental distress } \\
\text { (yes/no) K6 score of }>=5\end{array}$ & $43 \%$ & $44 \%$ & $29 \%$ & $29 \%$ \\
\hline \multicolumn{5}{|c|}{$\begin{array}{l}\text { Sub-set of only adults who are lesbian and gay to compare between those married/legally- } \\
\text { partnered and otherwise single }\end{array}$} \\
\hline Number of respondents & 279 & 1280 & 2300 & 3140 \\
\hline $\begin{array}{l}\text { Psychological distress mean and standard deviation } \\
\text { Kessler 6-item (K6) continuous score (0-24) }\end{array}$ & $\begin{array}{l}5.09 \\
(0.38)\end{array}$ & $4.14(0.22)$ & $\begin{array}{c}4.81 \\
(0.14)\end{array}$ & $5.40(0.17)$ \\
\hline $\begin{array}{l}\text { Percent reporting at least moderate mental distress } \\
\text { (yes/no) K6 score of }>=5\end{array}$ & $50 \%$ & $33 \%$ & $43 \%$ & $48 \%$ \\
\hline $\begin{array}{l}\text { Conditional adjusted odds ratio \& 95\% CI of moderate } \\
\text { mental distress } \\
\text { Post-CSCD vs. Pre-CSCD }\end{array}$ & & $\begin{array}{l}0.52(0.33 \\
\quad 0.82)\end{array}$ & & $\begin{array}{l}1.04(0.85 \\
1.29)\end{array}$ \\
\hline
\end{tabular}

Table 3.

Mean scores of psychological distress and percent reporting moderate mental distress pre and post the 2008 California supreme court decision (CSCD) to legalize same-sex marriage: CHIS data 2005-2015.

experiencing at least moderate psychological distress by sexual identity, gender and marital status from 2005 through 2015. Joinpoint analyses were conducted separately for sub-groups by gender, marital status and sexual identity. Distress levels remain relatively stable over time among married and other heterosexual women and among married heterosexual males. Moderate distress percentages dip in 2013 among married/partnered lesbians and gays as a group (lesbians and gays) and 

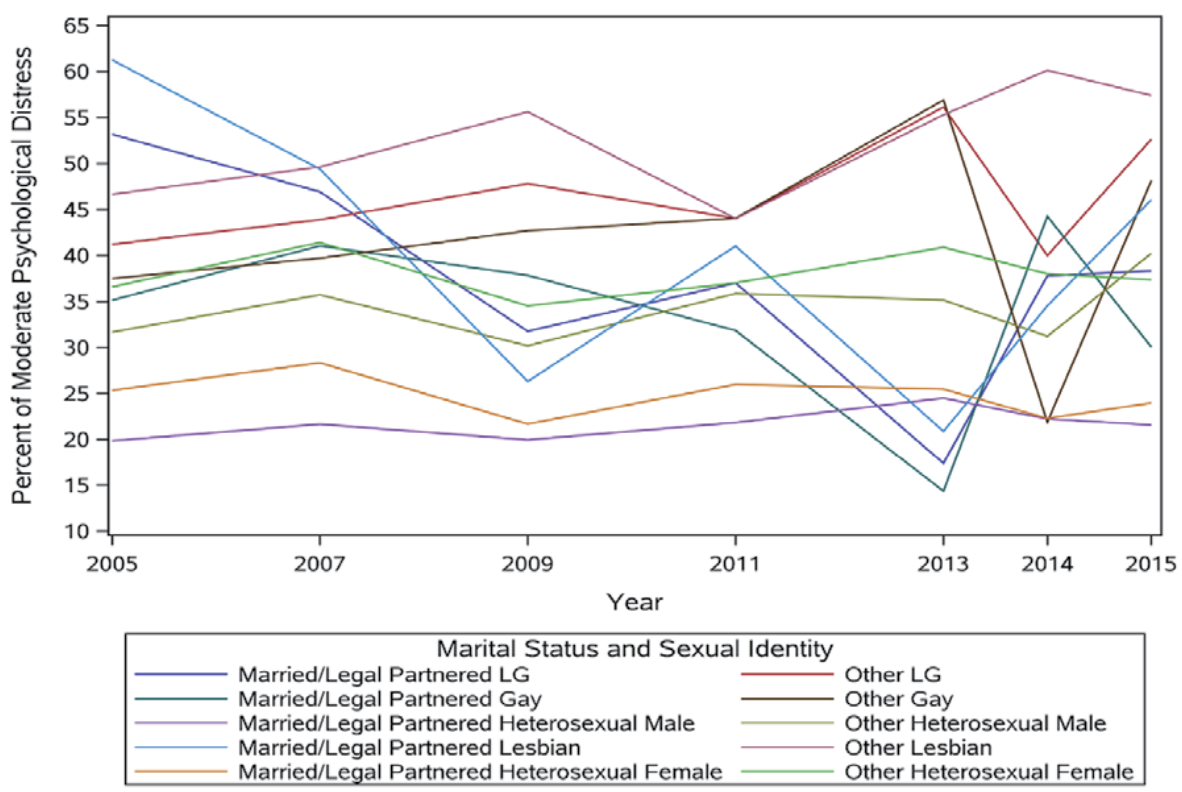

Figure 1.

Trend of moderate phycological distress by marital status and sexual identity.

separately (married/partnered gays, married partnered lesbians). Distress increased in this same year among other gay men and lesbian women. Distress scores increased from their 2013 levels among married/partnered lesbians and gays in 2014 and decreased only among gay men in 2015, the year of the U.S. Supreme Court decision that legalized same-sex marriage. However, none of these trends were significant as determined by the $\mathrm{p}$-values for each slope in Joinpoint analyses. In addition, there was no significant changing point in terms of year for any of the slopes for moderate psychological distress for any of the sub-groups.

\section{Discussion}

Before same-sex marriage became legal throughout the United States in 2015, clinical researchers promoted marriage equality as a health promotion strategy for lesbian/gay women and gay men $[8,12,28]$. Others ventured that marriage equality not only would improve lesbian/gay health, but also would benefit society at large [29]. Though our project was more circumscribed, we found some confirmation of our exploratory aim: Many adult Californians in legal same-sex partnerships and marriages reported lower levels of psychological distress than their single counterparts following the CSCD.

The inelegant results in this quasi-natural experiment may be no surprise, as this time period was fraught with instability regarding the state legality of same-sex marriage/partnerships and the stay of issuing same-sex marriage licenses between November 2008 and June 2014. Though Californians in same-sex unions maintained their legal status at the ends of the disputes, during this tumultuous legal period some undoubtedly worried whether their marital status would continue. Engaged same-sex couples were precluded from marriage licenses. Those with same-sex orientations may have experienced stressors related to passage of the state referendum Proposition 8 and the court cases appealing that referendum that abolished marriage for same-sex couples. In sum, this period was uncertain on the 
status of same-sex marriage and mental health self-reports fluctuated during periods when the issue's status also changed (Figure 1, Table 3). Reports of distress declined over time for legally-married or -partnered lesbian women and gay men while distress increased for their single counterparts. However, the changes in slope were not significant and no single year showed as the changing point when using Joinpoint analyses. In contrast, distress reports among heterosexual women and men remained relatively stable between 2009 and 2015. Our results suggest that marriage may have had a positive influence on mental health for legally-married and -partnered gay and lesbian people even during this turbulent period.

Recent studies posit that people in legal same-sex relationships have higher relationship stability, more financial resources, and better health outcomes than couples who cohabit without legal recognition $[19,30]$ and that marriage may mitigate minority stress effects among same-sex and other marginalized couples [21]. Our project found lower psychological distress levels among many lesbian/gay women and gay male Californians, though this health benefit was not uniform over time across same-sex marriages or legal partnerships-perhaps a reflection of the time period during which the continued legality of same-sex marriage in California was uncertain. The support we found for our hypothesis, even when the data were collected during a period in which the legality of same-sex marriage was questioned, reinforces a finding of a National Academies report that encourages research to understand the qualities of resilience unique to sexual minorities and how that relates to their overall health [3].

We also found evidence that gay and lesbian CHIS respondents who were legally-married and -partnered were substantively more likely to be employed and to have college educations than those unmarried or not legally partnered. That said, marriage equality in California can find its roots in 2005 legislation that required private employers to extend health insurance benefits to employees' same-sex partners just as the benefits were extended to opposite-sex spouses-a time when samesex marriage was unlawful. An earlier study found this policy had no influence on gay men but was of great benefit to lesbian women [17]. Our work, in light of previous studies, suggests more research is needed to explore whether and how same- and opposite-sex marriage is associated with benefits to health, well-being, and prosperity across communities.

Minority stress theory posits that prejudicial experiences over the lifecourse have a negative impact on the actual and perceived mental and physical health of lesbian/gay people [18, 21, 31]. Full legal protections for sexual- and genderminorities are incomplete; however, an exploration of the influence of national marriage equality on health issues of the multifaceted, non-exclusively-heterosexual, cis- and transgender people who comprise sexual minorities may be worthwhile.

Studies using future iterations of CHIS can determine whether the mental health benefits we found continue over time and whether other self-reported health benefits emerge. For example, extant research suggests that marriage equality has, at minimum, mental health benefits for non-heterosexual youth for whom suicide is the second most frequent cause of death [32-34].

Additional research projects might explore these questions across U.S. populations beyond California. To explore the implications of minority stress theory more thoroughly, future projects might consider biopsychosocial measures typically associated with stress responses-for example, to explore changes or differences in telomere lengths [35] and/or cortisol levels [36] in addition to self-reported data from single and married lesbian/gay women, gay men, and additional sexual and gender minorities over time. Longitudinal studies in this regard would enhance both understanding and health promotion among sexual minorities. 


\subsection{Limitations}

First, as CHIS is a continuous cross-sectional study, our findings suggest a trend in the populace rather than a change in a discrete set of Californians. Second is that legal partnerships and legal marriage did not convey identical rights and privileges between 2005 and 2008, a period that included concurrent, limited periods of cityand county-based same-sex marriages in California. Moreover, litigation precluded issuance of marriage licenses to same-sex couples between November 2008 and June 2013. Disparate results among legally-partnered, married, and other gay male and lesbian Californians perhaps reflect that uncertainty. Third, we were unable to report results separately by legal same-sex marriage and partnership between 2013 and 2015 though our results nonetheless appear to correspond with the historical events related to this issue during the period. Fourth is the need to exclude bisexually-identified Californians because the survey's order and skip pattern complicate notions of selfidentification and self-report of sexual behavior. For example, a bisexually-identified respondent who reported no sex with a same-sex partner in the last 12 months would not have been asked the question of same-sex legal partnership/marriage. Additionally, the partner's sex/gender and sexual orientation were not reported.

This fourth limitation demonstrates that a respondent's reported sexual identity is not necessarily equivalent to that respondent's sexual behavior or to the sex/ gender of that individual's spouse-particularly in our era of increasingly fluid sexual and gender identities [37-39] and growing researcher attention to the intersectionality framework to integrate the complexity of individual lived experiences within efforts to improve care and research in health and well-being [40-42]. For a more comprehensive understanding of the influences of sexual identity, gender identity, and marital status on human health and well-being, survey questions may elicit not only the sex/gender identity of a respondent but also of the respondent's spouse, for example, to help determine the influence of same- or opposite-sex marriage on the health of bisexual or transgender people.

Researchers adapting CHIS to account more precisely for same-sex marriage influences could follow the current question on "now married, living with a partner in a marriage-like relationship, widowed, divorced, separated, or never married" [43] by asking whether the spouse/partner referenced in the previous question is the same or opposite sex as the respondent.

There are thorough conversations across and outside the academy that will lead to comprehensive revisions of survey methodologies to measure the identity and behavior of respondents and their respective spouses. In the interim, the California Health Interview Survey (CHIS) provides a best practice on how to design and adapt questions to collect data that can explain the influence of legal marriage and partnerships on health and well-being, including sex/gender and sexual orientation identities [1]. Research and surveillance methodologies occasionally must respond quickly to provide data-driven public health recommendations. This study demonstrates CHIS's ability to explore the health impact of marriage for same-sex couples, and a need for survey questions to elicit information about marital status and the sex/gender of a respondent's spouse inclusive of sexual identities. Such collection is critical for data-driven health recommendations as sexual and gender identities become increasingly fluid and nuanced.

\section{Acknowledgements}

The UCLA Center for Health Policy Research conducts the California Health Interview Survey (CHIS). The National Institutes of Health (NIH) Office of 
Behavioral and Social Sciences Research (OBSSR), the NIH Office of Research on Women's Health (ORWH), and the National Cancer Institute (NCI) provided financial support for the design and implementation of previous iterations of CHIS. OBSSR and NCI supported access to the dataset created and analyzed for this project.

\section{Author Disclosure Statement}

No competing financial interests exist.

\section{Disclaimer}

This article represents only the authors' views and perspectives, not the positions of the National Institutes of Health or the U.S. Government.

\section{Author details}

William N. Elwood ${ }^{1 *}$, Veronica L. Irvin ${ }^{2}$, Benmei Liu ${ }^{3}$, Richard Lee ${ }^{4}$ and Nancy Breen ${ }^{5}$

1 Office of Behavioral and Social Sciences Research, National Institutes of Health, Bethesda, MD, United States

2 College of Public Health and Human Sciences, Oregon State University, Corvallis, OR, United States

3 Statistical Research and Applications Branch, National Cancer Institute, National Institutes of Health, Bethesda, MD, United States

4 Information Management Services, Inc., Rockville, MD, United States

5 National Institute on Minority Health and Health Disparities, National Institutes of Health, Bethesda, MD, United States

*Address all correspondence to: william.elwood@nih.gov

\section{IntechOpen}

(C) 2020 The Author(s). Licensee IntechOpen. This chapter is distributed under the terms of the Creative Commons Attribution License (http://creativecommons.org/licenses/ by/3.0), which permits unrestricted use, distribution, and reproduction in any medium, provided the original work is properly cited. (cc) BY 


\section{References}

[1] Federal Interagency Working Group on Improving Measurement of Sexual Orientation and Gender Identity in Federal Surveys. Toward a Research Agenda for Measuring Sexual Orientation and Gender Identity in Federal Surveys: Findings, Recommendations, and Next Steps. 2016. Washington: Federal Committee on Statistical Methodology. Available from: https://nces.ed.gov/FCSM/pdf/ SOGI_Research_Agenda_Final_Report_ 20161020.pdf [Accessed: 17 June 2019]

[2] Healthy People 2020. Lesbian, gay, bisexual, and transgender health. Washington: U.S. Department of Health and Human Services, Office of Disease Prevention and Health Promotion. 2020. Available from: https://www.healthype ople.gov/2020/topics-objectives/topic/ lesbian-gay-bisexual-and-transgenderhealth [Accessed: 18 June 2019]

[3] Institute of Medicine Committee on Lesbian, Gay, Bisexual, and Transgender Health Issues and Research Gaps and Opportunities. The Health of Lesbian, Gay, Bisexual, and Transgender People: Building a Foundation for Better Understanding. Washington: National Academies Press; 2011

[4] Langin K. NSF moves to pilot LGBT questions on national workforce surveys. Science. 7 November 2018:1126 DOI: $10.1126 /$ science.caredit.aav9886

[5] Centers for Disease Control and Prevention. Surgeon General's call to action: "Community health and prosperity." Federal Register, 83, 4523-4524. 2018. Available from: https://www.federalregister.gov/docume nts/2018/09/06/2018-19313/surgeon-ge nerals-call-to-action-community-healthand-prosperity [Accessed: 17 June 2019]

[6] Boswell J. Same-Sex Unions in PreModern Europe. New York: Villard Books; 1994
[7] Foucault M. The History of Sexuality Volume 1: An Introduction(R. Hurley, trans). New York: Pantheon Books; 1978

[8] Kealy-Bateman W, Pryor L. Marriage equality is a mental health issue.

Australasian Psychiatry. 2015;23:540-543. DOI: $10.1177 / 1039856215592318$

[9] Ponce NA, Cochran SD, Pizer JC, Mays VM. The effects of unequal access to health insurance for same-sex couples in California. Health Affairs

(Millwood). 2010;29:1539-1548. DOI:

10.1377/hlthaff.2009.0583

[10] Waite LJ, Gallagher M. The Case for Marriage: Why Married People Are Happier, Healthier, and Better off Financially. New York: Doubleday, 2001.

[11] Supreme Court of the United States. James Obergefell et al., Petitioners. v. Richard Hodges, Director, Ohio Department of Health, et al. Docket No. 14-556. 2015. Available from: https:// www.supremecourt.gov/opinions/ 14pdf/14-556_3204.pdf [Accessed: 17 June 2019]

[12] Gonzales G. Same-sex marriage-A prescription for better health. New England Journal of Medicine. 2014;370: 1373-1376. DOI: 10.1056/NEJMp1400254

[13] Hatzenbuehler ML, O'Cleirigh C, Grasso C, Meyer K, Saffren S, Bradford J. Effect of same-sex marriage laws on health care use and expenditures in sexual minority men: A quasi-natural experiment. American Journal of Public Health. 2012;102: 285-291. DOI: $10.2105 /$ AJPH.2011.300382

[14] LeBlanc AJ, Frost DM, Bowen K. Legal marriage, unequal recognition, and mental health among same-sex couples. Journal of Marriage and Family. 
2018;80:397-408. DOI: 10.1111/ jomf.12460

[15] Riggle EDB, Rostosky SS, Horne SG. Psychological distress well-being, and legal recognition in same-sex couple relationships. Journal of Family Psychology. 2010;24:82-86. DOI: 10.1037/a0017942

[16] Tran LD. Moderate effects of samesex legislation on dependent employerbased insurance coverage among sexual minorities. Medical Care Research and Review. 2016;73:752-768. DOI: 10.1177/ 1077558715625560

[17] Buchmueller TC, Carpenter CS. The effect of requiring private employers to extend health benefit eligibility to samesex partners of employees: Evidence from California. Journal of Policy Analysis and Management. 2012;31: 388-403. DOI: 10.1002/pam.21603

[18] Everett BG, Hatzenbuehler ML, Hughes TL. The impact of civil union legislation on minority stress, depression, and hazardous drinking in a diverse sample of sexual-minority women: A quasi-natural experiment. Social Science and Medicine. 2016;169:180-190. DOI: 10.1016/j.socscimed.2016.09.036

[19] Elwood WN, Irvin VL, Sun Q, Breen N. Measuring the influence of legally recognized partnerships on the health and well-being of same-sex couples: Utility of the California health interview survey. LGBT Health. 2017;4: 153-160. DOI: 10.1089/lgbt.2015.0085

[20] California Health Interview Survey. CHIS 2009 Methodology Report Series: Report 4-Response Rates. 2011. Los Angeles, CA: UCLA Center for Health Policy Research. Available from: http:// healthpolicy.ucla.edu/Documents/Ne wsroom\%20PDF/CHIS2009_method4. pdf [Accessed: 17 June 2019]

[21] LeBlanc AJ, Frost DM, Wight RG. Minority stress and stress proliferation among same-sex and other marginalized couples. Journal of Marriage and Family. 2015;77:40-59. DOI: 10.1111/jomf.12160

[22] Cunliffe VT. The epigenetic impacts of social stress: How does social adversity become biologically embedded? Epigenomics. 2016;8: 1653-1669. DOI: 10.2217/epi-2016-0075

[23] Smyth JM, Sliwinski MJ, Zawadzki MJ, Scott SB, Conroy DE, Lanza ST, et al. Everyday stress response targets in the science of behavior change. Behaviour Research and Therapy. 2018;101:20-29. DOI: 10.1016/j.brat.2017.09.009

[24] Institute of Medicine. Collecting Sexual Orientation and Gender Identity Data in Electronic Health Records: Workshop Summary. Washington: National Academies Press; 2013

[25] Kessler RC, Green JG, Gruber MJ, Sampson NA, Bromet E, Cuitan M, et al. Screening for serious mental illness in the general population with the $\mathrm{K} 6$ screening scale: Results from the WHO world mental health (WMH) survey initiative. International Journal of Methods in Psychiatric Research. 2010; 19(suppl 1):4-22. DOI: 10.1002/mpr.310

[26] Kessler RC, Andrews G, Colpe LJ, Hiripi E, Mroczek DK, Normand SL, et al. Short screening scales to monitor population prevalences and trends in non-specific psychological distress. Psychological Medicine. 2002;32: 959-976. DOI: $10.1017 /$ S0033291702006074

[27] Prochaska J, Sung H, Max W, Shi Y, Ong M. Validity study of the K6 scale as a measure of moderate mental distress based on mental health treatment need and utilization. International Journal of Methods in Psychiatric Research. 2012; 21:88-97. DOI: $10.1002 / m p r .1349$

[28] Campion EW, Morrisey S, Drazen JM. In support of same-sex 
marriage. New England Journal of Medicine. 2015;372:1852-1853. DOI: 10.1056/NEJMe1505179

[29] Rauch J. Gay Marriage: Why it Is Good for Gays, Good for Straights, and Good for America. New York: Times Books; 2004

[30] Wight RG, LeBlanc AJ, Badgett MVL. Same-sex legal marriage and psychological wellbeing: Findings from the California health interview survey. American Journal of Public Health. 2013;103:339-346. DOI: 10.2105/ AJPH.2012.301113

[31] Holman EG. Theoretical extensions of minority stress theory for sexual minority individuals in the workplace: A cross-contextual understanding of minority stress processes. Journal of Family Theory and Review. 2018;10: 165-180. DOI: $10.1111 /$ jftr.12246

[32] Di Giacomo E, Krausz M, Colmegna F, Aspesi F, Clerici Y, M. Estimating the risk of attempted suicide among sexual minority youths: A systematic review and meta-analysis. JAMA Pediatrics. 2018;172:1145-1152. DOI: 10.1001/jamapediatrics.2018.2731

[33] Kann L, Olsen EO, McManus T, Harris WA, Shanklin SL, Flint KH, et al. Sexual identity, sex of sexual contacts, and health-related behaviors among students in grades 9-12: United States and selected sites, 2015. Morbidity and Mortality Weekly Report. 2016;65: 1-102. DOI: 10.15585/mmwr.ss6509a1

[34] Raifman J, Moscoe E, Austin SB, McConnell M. Difference-in-differences analysis of the association between state same-sex marriage policies and adolescent suicide attempts. JAMA Pediatrics. 2017;17:350-356. DOI: 10.1001/jamapediatrics.2016.4529

[35] Montpetit AJ, Alhareeri AA, Montpetit M, Starkweather AR, Elmore LW, Filler K, et al. Telomere length: A review of methods for measurement. Nursing Research. 2014; 63:289-299. DOI: 10.1097/ NNR.0000000000000037

[36] Hengge UR, Reimann G, Schäfer A, Goos M. HIV-positive men differ in immunologic but not catecholamine response to an acute psychological stressor. Psychoneuroendocrinology. 2003;28:643-656. DOI: 10.1016/ S0306-4530(02)00048-3

[37] Chen MJ, McCann-Crosby B, Gunn S, Georgiadis P, Placencia F, Mann D, et al. Fluidity models in ancient Greece and current practices of sex assignment. Seminars in Perinatology. 2017;41:206-2013. DOI: 10.1053/j.semperi.2017.03.014

[38] Katz-Wise SL, Hyde JS. Sexual fluidity and related attitudes and beliefs among young adults with a same-gender orientation. Archives of Sexual Behavior. 2015;44:1459-1470. DOI: 10.1007/s10508-014-0420-1

[39] Oswalt SB, Evans S, Drott A. Beyond alphabet soup: Helping college health professionals understand sexual fluidity. Journal of American College Health. 2016;64:502-508. DOI: 10.1080/ 07448481.2016 .1170688

[40] Hankivsky O. Women's health, men's health, and gender and health: Implications of intersectionality. Social Science and Medicine. 2012;74: 1712-1720. DOI: 10.1016/ socscimed.2011.11.029

[41] Warner LR. A best practices guide to intersectional approaches in psychological research. Sex Roles. 2008;59:454-463. DOI: $10.1007 /$ s11199-008-9504-5

[42] Wilson Y, White A, Jefferson A, Danis M. Broadening the conversation about intersectionality in clinical medicine. American Journal of Bioethics. 2019;19:W1-W5. DOI: 10.1080/15265161.2019.1574318 
Psychological Health Influences of Legal-Marriage and-Partnerships on Same-Sex Couples DOI: http://dx.doi.org/10.5772/intechopen.90249

[43] California Health Interview Survey.

CHIS 2018: Adult questionnaire,

Version 1.52. 2019. Los Angeles: UCLA

Center for Health Policy Research.

Available from: https://healthpolicy.

ucla.edu/chis/design/Documents/2018\%

20Questionnaires\%20and\%20Topics\%

20List/CHIS\%202018\%20ADULT.pdf

[Accessed: 17 June 2019] 



\title{
"Kidnapping the Bride"-A Traditional Sasak Wedding Seen in Sesak Cinta Di Tanah Sasak Novel: A Model in Contemporary Indonesian Literature Studies
}

\author{
H.D. Dharma Satrya, Faruk Faruk and Pujiharto Pujiharto
}

\begin{abstract}
This article aims to create dialog of "kidnapping the bride" reality in several studies and to construct the practice in reality. Discussion of "kidnapping the bride" practices in literature and cultural studies of Lombok intends to find contemporary Indonesian literary models of ethnic Lombok. To achieve this goal, this article applies Stuart Hall's representation theory. In literary studies, "kidnapping the bride" is constructed as a critique of nobility. Sesak Cinta di Tanah Sasak novel construct "kidnapping the bride" as a criminal act. The meaning is constructed by Islamic discourse and tradition (nobility). Resistance to the discourse is what presents a romantic discourse. Romantic discourse, in the novel When Love Takes to Go, is constructed to fight against feudalism and capitalism. Romantic discourse in Opto Ergo Sum is a tradition discourse. The discourse is gathered from different voices, men's and women's voices. Women's voice tends to be strong to the lid of the real world (in Ketika Cinta Tak Mau Pergi). Man's voice rejects integration (in Opto Ergo Sum). Finally, the study of Sesak Cinta di Tanah Sasak and two other novels signifies a model for contemporary Indonesian literary studies. The model can be found by blurring the boundaries between Lombok cultural study and Indonesian literature.
\end{abstract}

Keywords: practice of "kidnapping the bride", Indonesian novel Contemporer, discourse, Lombok (cultural studies)

\section{Introduction}

"Kidnapping the bride" of Sasak wedding practices puts as a record by the Ministry of Education and Culture as Lombok West Nusa Tenggara customs [1]. This practice is also supported by the government as a code of conduct of Sasak people [2]. Lukman [3] strengthens the position of "kidnapping the bride" in Sasak traditional culture. The practice of "kidnapping the bride," from the independence era until Order Baru era, is discussed as a traditional Sasak wedding. The practice of "kidnapping the bride" as a Sasak cultural identity is voiced by the noble group, a heterodox Islamic group. After Order Baru period, "kidnapping the bride" discourse got its match from orthodox Islamic groups. This shows that there is a change in 
representation in constructing the practice of "kidnapping the bride." The change in representation of the practice of "kidnapping the bride" of Sasak was caused by the election of the master teacher to the West Nusa Tenggara region. The previous regional head, in the 2003-2008 period, came from a noble group, Lalu Serinata.

"Kidnapping the bride" in the master master's regime is adjusted to Islamic law and state law [4]. In this context of change, the practice of "kidnapping the bride" is important to be thorough.

The practice of "kidnapping the bride" serves an important theme of the Indonesian novel Lombok. The theme is firstly portrayed by Nadira Khalid in the novel Ketika Cinta Tak Mau Pergi. The novel delineates romance to counter feudalism and capitalism [5]. This practice in the Sesak Cinta di Tanah Sasak is constructed as a criminal act by aristocrats and police officers. Romance is the only way. Romantic discourse wins the fight. Ketika Cinta Tak Mau Pergi positions stronger to the poles of the real world, even if it does not experience unification. Sesak Cinta di Tanah Sasak maintains stronger to the ideal world, because Baiq Raihan dies. Ketika Cinta Tak Mau Pergi, position the nobility as the poor people and the ranks of corals as the people are rich. Sesak Cinta di Tanah Sasak perfoms the nobility as rich people and the ranks of coral remained as poor people.

Both novels represent the practice of "kidnapping the bride" differently. Ketika Cinta Tidak Mau Pergi novel does not involve the police to resolve Sahnim's parents' disapproval of "kidnapping the bride" by Lalu Kertiaji. Sesak Cinta di Tanah Sasak novel involves the police as judges for "kidnapping the bride" considered as a criminal act. Nagara cooperates with the oppressing nobility of the people, the ranks of coral. The difference in "kidnapping the bride" representation in the two novels is caused by the socio-political context that lies behind it. Ketika Cinta Tidak Mau Pergi was published in the socio-political situation of the aristocratic regime (2003-2008), while Sesak Cinta di Tanah Sasak presents in the socio-political context of "tuan guru" regime, 2008-2018.

Some studies [6-10] reveals that ethnic representation in contemporary Indonesian literary studies is assumed to be a local wisdom. These studies ignore the political movements of local people who at some stage are no longer local. Ethnic writers in the United States carry out social and political movements so that ethnic groups have important positions, both in literature and politics [11]. Lombok experiences this situation. From independence until the early reformation era, Lombok is led by a governor from Java. It is only in 2003 that the native son is elected as governor. Sasak ethnic identity has begun to emerge in the 2000s. The Indonesian literary production of Lombok is part of an ethnic revival to sound Sasak cultural identity.

Indonesian Literature of Lombok emerges as a meeting point of two studies, namely Lombok studies and contemporary Indonesian literary studies. Novel Sesak Cinta di Tanah Sasak is in these two studies. The Lombok study sees "kidnapping the bride" as a reality that continues to be constructed in various perspectives, for example Islamic studies, legal studies, and cultural studies. The reality of "kidnapping the bride" is constructed according to the perspective used to see it. Contemporary Indonesian literature studies see "kidnapping the bride" as part of romantic discourse [5]. Sesak Cinta di Tanah Sasak reinforces the position of "kidnapping the bride" as a romantic discourse. This practice keeps him trapped in law and prison. All that is left is the ideal world. The tendency to achieve unification is weak. Romantic discourse in the novel Ketika Cinta Tidak Mau Pergi and Sesak Cinta di Tanah Sasak does not differ from romantic discourse in modern Indonesian literature in case of Balai Pustaka. Romantic discourse in Balai Pustaka tradition creates unification, but it does not reach unification, while romantic discourse in the Opto Ergo Sum novel consciously rejected unification [12]. 
"Kidnapping the Bride"-A Traditional Sasak Wedding Seen in Sesak Cinta Di Tanah Sasak... DOI: http://dx.doi.org/10.5772/intechopen.93697

Ethnic representation in contemporary Indonesian literature with the Lombok case can be used as a model in contemporary Indonesian literature and a model for Lombok (cultural) studies. In this context, the boundary between the contemporary Indonesian and Lombok literature is blurred.

\section{Methodology}

This article applies Stuart Hall's representation theory. Hall's representation theory serves to theorize the practice of Lombok Sasak "kidnapping the bride." It accommodates the context of contemporary Indonesian literature with Lombok as its ethnic issue. This practice also delivers a sign and a discourse. As a sign, "kidnapping the bride" is constructed as semiotic analysis in the novel. As a discourse, "kidnapping the bride" is constructed academically by several studies. The novel serves as primary data source while some studies of "kidnapping the bride" and discourse about Lombok supplies secondary data sources. The first data source is in the form of lingual units in the form of sentences or paragraphs that provide information about the practice of "kidnapping the bride." The second data source is in the form of discursive statements from several studies of "kidnapping the bride" and Lombok. The data source delivers the perspective of Sasak people living in the Sasak culture of Lombok. The first and second analysis applies semiotic analysis and discourse. The analysis conveys the experience of researchers as Sasak people taken from the researcher's experience. The meaning of "kidnapping the bride" in a novel is as a dialog in academic discourse.

\section{Discussion}

\subsection{Running away in literary studies, cultural studies, and Islamic (law) studies}

The "kidnapping the bride" study conducted by Wijaya [13] and Zuhairini [14] positions "kidnapping the bride" as a culture that needs to be criticized. Both interpret Merpati Kembar di Lombok novel as criticism to the nobles. Wijaya sees "kidnapping the bride" as a critic of the class, while Zuhairini sees it in the context of women. Wijaya interpretes the novel as based on Islamic teachings, while Zuhairini was based on a modern way of thinking with the theme of women's freedom to behave. Wijaya uses a religious perspective, while Zuhairini uses a feminist perspective.

In cultural studies, the practice of "kidnapping the bride" was conceptualized as a result of acculturation of Sasak and Bali [15]. "Kidnapping the bride" is not a native Sasak culture, but acculturation with Balinese culture [4]. Therefore, the development of sees "kidnapping the bride" experiences codification with Islamic and state law. Lukman [16] sees "kidnapping the bride" from a legal perspective. According to him, there are three laws in the "kidnapping the bride" tradition, namely religious, customary and state law ([16], 427). In the perspective of Islamic law, Basriadi [17] reviews marital law of different classes, aristocratic women with non-aristocratic men, not contrary to customary law. If in practice, there are parents who do not want to marry off their children to men of different classes, then the bride and groom are married by the guardian of the judge. Aminullah [18] explaines that the practice of "kidnapping the bride" has been modified, for example in the tradition of drinking alcohol in the process of collusion. Mahayanti [19] sees the tension of Islamic law and customary law in terms of giving pisuke (money). In customary law, pisuke must be given, whereas in Islamic law is not 
touched other than dowry. The reality of eloping practices varies according to the perspective of the study.

\section{2 "Kidnapping the Bride" Discourse}

Krulfeld [20] distinguishes Sasak society based on the type of religiosity. First, the Sasak Wetu Telu type community, orthodox Islam. Second, the Sasak people of type five, orthodox Islam. The first acts based on adat, while the second acts based on the commandments of God as stated in the book. The Sasak people experience a change from traditional religious types, adat, to orthodox Islamic religious types $[20,21]$. Budiwanti [21] shows how traditional Islam, wetu telu, is displaced into a marginalized group. Orthodox Islamic groups work together with the government to purify Islamic teachings [21, 22]. The battle between the two discourses was ongoing. Lukman [3] strengthens the Sasak tradition by emphasizing the village krame and Sorong Serah Aji Krama marriage customs. Suhardi [23] added Sasak custom by including ceremonies related to birth and death. Hadi [24] photographs the lives of Sasak people. In the portrait, Hadi [24] traces the distance of Lombok customs to the calculation of the lunar calendar in Sasak's mind. Custom or tradition as a discourse about Lombok is still being reproduced. This is the case with Islamic discourse. Islam provides a new color in Lombok culture. Islam and tradition or tradition are seen as dialectical [25]. Dialog with Islam constructs a modern Sasak identity [26].

Asnawi [27] emphasizes the position of the teacher as a refinement of Islamic teachings. Tuan Guru becomes the main agent of the acculturation of Islamic values in the Sasak culture [28]. The important position of tuan guru (master teacher) in Lombok culture and especially in Islamization determines how custom is contextualized with Islamic law. The important position of the teacher in politics in West Nusa Tenggara determines how custom is contextualized with state law. Macdougall [29], Kristiansen [30] and Kingsley [31] highlight the rise of religious groups in maintaining the security of the people in Lombok. Islamic discourse is not only constructed by Western scholars, but also by the West Nusa Tenggara region [32]. In the book NTB Bersaing, the local government discusses the Islamic Center as an icon of West Nusa Tenggara, as a center for Islamic activities [32]. In a historical dissertation written by Fajdri [33], Lombok as an Islamic society from the very beginning of its existence. Islamic discourse shifts the position of tradition in the production of scientific knowledge about Lombok and especially the practice of "kidnapping the bride." In the 2000s-2010s, Lombok was constructed as Islam. Thus, the practice of "kidnapping the bride" is adjusted to Islamic law [4]. The current construction of Lombok is dominated by Islamic discourse.

\subsection{The Noble “Tuan Guru” in Sesak Cinta di Tanah Sasak Novel}

This novel presents a noble man and tuan-guru in one character, Lalu Said. Baiq Raihan is betrothed with a nobleman and tuan guru.

As time passes from so many choices of Mamiqnya, Raihan is proposed by young nobles from the village of Gede. Handsome, handsome, smart young man. It is reported that the son of a Tuan Guru is certainly respected for having his recitatory congregants. The young man reportedly has completed his education in Arab and is expected to be able to return to his Islamic group representing the people and eventually control the people ([34], p. 14-15).

In Lombok culture, tuan guru (master teacher) as social status tend to be inherited [35]. Alaini [36] mentions that status is achieved which is open, while nobility 
"Kidnapping the Bride"-A Traditional Sasak Wedding Seen in Sesak Cinta Di Tanah Sasak... DOI: http://dx.doi.org/10.5772/intechopen.93697

is inherited status. Tuan guru's son is definitely tuan guru. The teacher prepares his child to be his successor. One way the child is prepared is to send them to Arabic. Returning from Arab, the child will be introduced to the congregation of his parents as well as being introduced as his successor. Tuan guru has the same position as the noble man, that is, the figure who is equally respected by the community. However, Lalu Said and his parents have a double honor as tuan guru and nobleman. Tuan guru has a high social status in Sasak community structure to match the high position of the aristocracy. Such conditions make Raihan's father insist on pairing his child with Lalu Said, because of the aristocracy and tuan guru.

\subsection{Betrayed love}

Raihan is forced to choose and accept an arranged marriage with Lalu Said. The choice is taken in order to maintain the honor of his family. That choice is a manifestation of Raihan's disdain for her love for Habib. In order to maintain that honor, Raihan choses to betray Habib's love, an ordinary man, jajarkarang.

"Look, sir, if we want to make peace with our family, this father as Habib's parents, must take responsibility for several legal agreements, and sanctions if they are violated. Then, Raihan is asked to tell about her journey, but it is strange to Habib that what is told. It cannot help him to get out from the legal problem, because Raihan's testimony is not in favor of him, in the love they were fighting for. Shed tears of sadness. How have our fathers heard the confession of this girl, then is there anyone capable of defending Habib? "That's not right sir, that's wrong, everything she says is her parents' orders. ".

“Calm down!” The policeman snapped.

"Sir! How about the word peace we have discussed earlier, "asked his father. "Hearing all the stories and confessions of our girls is enough to get here and we don't need peace money from you guys!"

"Alright sir, whatever the responsibility I am ready, even though I have to be in jail. Allah is all-knowing with all this and you calm down, all of this I will pass with sincerity and patience and you forgive me please take care of his health, again sorry, sir, I have not been able to be a filial son. Then Habib kisses the soles of her father's feet. The wall clock at the police station tinkles as Habib stepps into a detention car for safekeeping to the police station, going through his days until the sentence is completed ([34], p. 47-48).

Raihan gives false testimony, so the act of carrying Raihan serves as a criminal act. Though both of them agree to elope to avoid arranged marriages Raihan with Lalu Said, the son of the noble tuan guru. Raihan's father reports to the police about the kidnapping of Raihan. Police gives a hand to search for Raihan. Raihan dan Habib are found and then taken to the police station. Raihan is forced to give false testimony in order to maintain the honor of her family. In that case the police only accepts Raihan's decision and testimony as a victim who is kidnapped. Raihan's attitude who chooses to give false testimony feels suffering to the man she loves, because Habib is imprisoned.

Raihan's love for Habib is now a mere lie that he must throw away in his mind. However, all that is useless, it's so hard for him to just forget it all. The betrayal of love and disappointment he gave is the most beautiful gift that is sweet at first, then 
bitter at the end. His best friend Nasir visits him that afternoon after a few days ago came to him. This time Nasir arrives early. From the jail, Habib comes out in a white prisoned costume bearing number 07 . The iron door is opened by officers then Habib comes out with a listless face ([34], p. 49).

The quote shows that Habib finally becomes a prisoner. His love has taken him to prison. The love he believes has become a lie. Betrayal love is the most beautiful gift, because that's all that's left. After the disappointment there is no chance to change the betrayal. What remains is memories. Betrayal is not without consequences for Raihan himself. Both are imprisoned. Habib is imprisoned in the jail and Raihan is imprisoned by tradition. Both prisons are equally limiting their actions. But the prison occupied by Raihan is a prison of eternal tradition in his life. The prison that is occupied by Habib is a prison that has physical strength, while Raihan is a prison that has power that is not only physical. Faruk [37] called it a prison because of formal normative or ethical legal issues, while the prison occupied by Raihan is cultural and philosophical because it involves the suppression of freedom of choice. Habib can only come out after he has lived according to established rules, while Raihan can only get out of traditional prison after his family dies and his marriage ends in divorce.

Her face is covered with sorrow, her thin body told a bitter truth. She sits not in her husband's bed, she does not live in her in-laws' house but she is in her house. Raihan is now veiled in gloom, she returns to her umma while her mother has passed away for a year. She returned home with full of regret and accepted his new status as a widow, lying. Guilt and betrayal of love still make an impression in her heart. Her beauty is faded even though there are still beautiful lines on her face. The blue eyes look sad, while his heart continues to whimper with prolonged injury $([34], 66)$.

Raihan divorces her husband because she is unable to give her husband offspring. Raihan is widowed. Her widowed status is unable to get her out of suffering. She still feels hurt, sorry, and guilty. Her disdain for Habib still leaves an imprint on her heart and hurt him all his life before he received an apology from Habib. Her injured heart is followed by shortness of breath and lung cancer. She dies after meeting with Habib.
Shortness of breath and cancer undermines her lungs, Raihan is only able to lie on the bed, breathing hard. Ummi in surrender faithfully goes side by her side. While being close to her death, the verses of the Koran from the Surah Yasin continue to be recited by her family and close relatives. Her curling eyelashes look wet as she endures pain, the patient and faithful rays of her face radiate happily in the arms of her beloved Habib. The howling sound of dogs gripped together with a smile that finally silenced then the tired body stiffened. Her mom cries helplessly staring at her only daughter silently without a sound. Innalillahiwainnailaihirajiu. The fragrant breath has now stopped. News of the death of Baiq Raihan along with fragrance and joy was always implied on his face. And for those who know her feel great loss and sadness. While Habib, a desperate lover, cannot hold back tears of pain, his heart seems to be cut into thorns, it hurt and hurt when he compares the time in the jail. After seven years of separation and seven months he feels the meeting without any restrictions. Now it turns out she has gone to face the divine ([34], p. 72).

Raihan visits to the divine in accordance with her promise. She passes away after meeting with Habib. The meeting is at once a farewell for both. 
"Kidnapping the Bride"-A Traditional Sasak Wedding Seen in Sesak Cinta Di Tanah Sasak... DOI: http://dx.doi.org/10.5772/intechopen.93697

\section{5 “Kidnapping the bride” discourse in Sesak Cinta di Tanah Sasak Novel}

"Kidnapping the bride" done by Habib and Baiq Raihan is not a custom event, but an abduction event. Therefore, "kidnapping the bride" is a criminal case. The novel positions this practice as a criminal act. Raihan is a victim and a witness who confirms that Habib kidnappes her. Though they love each other and they make the decision to do this practice together. If the novel positions Raihan in favor of Habib, then "kidnapping the bride" is a process of the Sasak wedding tradition. Habib is put in jail for kidnapping. Raihan testifies that she is kidnapped. Sesak Cinta di Tanah Sasak sided with the aristocrats and masters of the teacher as well. His side is not about placing "kidnapping the bride" as a custom that must be maintained, but customs that have the potential to become criminal acts. The custom considered as high esteem assigns the matchmaking custom in the Islamic tradition. Kyai (and) tuan guru work together to position "kidnapping the bride" as a tradition that no longer contains goodness. The practice of "kidnapping the bride" is seen as a practice that needs to be avoided. Thus, the practice of "kidnapping the bride" is a threat to the group of nobles and tuan guru (master teacher). It assumes that the practice of "kidnapping the bride" is in two traditions, namely the aristocracy and the folk traditions.

Sesak Cinta di Tanah Sasak is constructed by tradition discourse (nobility) and Islamic discourse. The two discourses worked together to form Sasak identity. Sasak traditions (aristocrats) are represented by groups of aristocrats and Sasak Muslims who are represented by Sasak masters of the teacher. The construction determines who can take eloping and who may not. The practice of "kidnapping the bride" can only occur with a pair of lovers who have the same status, for example both aristocrats and or aristocratic men with non-aristocratic women. Often the practice of "kidnapping the bride" does not end with marriage if it is related to marriages of different social status, for example in the case of Habib and Baiq Raihan. The "kidnapping the bride" tradition can only be done by those who are of the same social status. Tradition in that sense is tradition in the perspective of nobility.

\subsection{Lombok Indonesian literature: A study in Indonesian literature}

Indonesian literature as a scientific discipline develops several paradigms in its studies of Indonesian literary works. The study of Indonesian literature in the 1990s to 2000s was dominated by structuralism [38]. Supriyadi saw the tendency of Indonesian literary studies at two universities, namely Gadjah Mada University and the University of Indonesia. Both universities represent the paradigm of Indonesian literary studies. Faruk [39] offers several paradigms in the study of Indonesian literature, for example humanist literary criticism, structuralist literary criticism, post-structural literary criticism, post -amarxist literary criticism, cultural studies and critical approaches. Faruk offers these paradigms in the context of providing various possible paradigms in Indonesian literature. The paradigm is born from literary studies which at a certain level becomes a paradigm.

The paradigm in Kuhn is a model [40]. Ahimsa-Putra distinguishes paradigms and models. For Ahimsa-Putra [41], the model is part of the paradigm. The model is defined as a parable of the object [41]. Both can be used to formulate contemporary Indonesian literary study models. In the context of combining the concepts of Kuhn and Ahimsa-Putra, Saukko's concept provides an alternative. Saukko [42] offers four paradigms in cultural studies, namely triangulation, prisms, semiotic material, and dialog. All four applies different metaphors to describe reality. Saukko's concept can be used to see models in contemporary Indonesian literature. 
Romantic discourse in the novel Sesak Cinta di Tanah Sasak is confronted with Islamic discourse and tradition (nobility). Islamic discourse and tradition of constructing "kidnapping the bride" as a criminal act, thus involving the police to resolve the case of eloping Baiq Raihan with Habib. Romantic discourse in the novel Ketika Cinta Tidak Mau Pergi, Opto Ergo Sum, and Sesak Cinta di Tanah Sasak voiced different interests. Romantic discourse in Ketika Cinta Tidak Mau Pergi is a criticism of feudalism and capitalism, while in Opto Ergo Sum is a critique of modernity. Meanwhile, the romantic discourse in Sesak Cinta di Tanah Sasak is a criticism of feudalism and Islam (orthodox).

Romantic discourse comes from a different sound. The first is a female voice and the second and third is a male voice. Women's romantic discourse tends to unite, while men's romantic discourse tends towards separation and unification. The study of the Indonesian novel Lombok in the third case of the Lombok novel is a model of contemporary Indonesian literary studies. The model can be used to see how ethnic representations in contemporary Indonesian literature with different ethnic cases.

Studies in Ketika Cinta Tidak Mau Pergi, Opto Ergo Sum, and Sesak Cinta di Tanah Sasak perform models or paradigms of contemporary Indonesian literary studies. Sesak Cinta di Tanah Sasak constructs the reality of "kidnapping the bride" which differs from Ketika Cinta Tidak Mau Pergi novel. Opto Ergo Sum novel constructs a love that refuses unification. The three of them voice love together. Sesak Cinta di Tanah Sasak and Ketika Cinta Tidak Mau Pergi try to unite. Love in Opto Ergo Sum delineates traditional discourse, just as Princess Madalika rejects the propositions of kings for the peace of her people [12]. It is done by Rahmat in Opto Ergo Sum.

\section{Conclusion}

The meaning of "kidnapping the bride" as dominant discourse of Lombok represents Islamic context. "Kidnapping the bride" practice in Islamic and State perspective positions as a tradition which needs to adapt with Islamic and state law. The meaning of "kidnapping the bride" in Sesak Cinta di Tanah Sasak is part of the discursive formation that Lombok is Islam and a tradition (nobility). In the text, the social position of the noble man makes Baiq Raihan betray Habib. Therefore, discourse of tradition and Islam creates its opponents. In that context, romantic discourse applies as resistance to Islamic discourse and tradition (nobility).

The practice of "kidnapping the bride" is commonly discussed in contemporary Indonesian literature. Sesak Cinta di Tanah Sasak and Ketika Cinta Tidak Mau Pergi novel are constructed as a romantic discourse in two perspectives, namely male and female perspectives. In the male perspective, romantic discourse tends to be stronger to the poles of the ideal world, while in the perspective of women, tend to be stronger to the poles of the real world. In the third perspective on the Opto Ergo Sum novel case, the perspective of a male jajar Karang (ordinary person), refuses to unite. In the third perspective, romantic discourse delivers the mindset of the Sasak tradition which prioritizes peace. In the myth of Madalika Princess for the sake of peace she did not choose one of the princes who proposed. The study of the Sesak Cinta di Tanah Sasak and two other Sasak novels (Ketika Cinta Tidak Mau Pergi and Opto Ergo Sum) are treated as models for contemporary Indonesian literary studies. This study seeks the dialog to the reality of "kidnapping the bride" and construct a non-singular reality of the practice. This study unifies and obscures the boundary between the study of Lombok culture and Indonesian literature. 
"Kidnapping the Bride"-A Traditional Sasak Wedding Seen in Sesak Cinta Di Tanah Sasak... DOI: http://dx.doi.org/10.5772/intechopen.93697

\section{Acknowledgements}

This article is part of my dissertation; therefore, I would like to express my great gratitude to the Directorate of Research and Community Service, Directorate General of Strengthening for Research and Development, the Ministry of Research, Technology and Higher Education who has funded this dissertation research.

\section{Author details}

H.D. Dharma Satrya ${ }^{1 *}$, Faruk Faruk ${ }^{2}$ and Pujiharto Pujiharto ${ }^{2}$

1 Universitas Hamzanwadi, Indonesia

2 Universitas Gadjah Mada, Indonesia

*Address all correspondence to: dharmasatryahd@gmail.com

\section{IntechOpen}

(C) 2020 The Author(s). Licensee IntechOpen. This chapter is distributed under the terms of the Creative Commons Attribution License (http://creativecommons.org/licenses/ by/3.0), which permits unrestricted use, distribution, and reproduction in any medium, provided the original work is properly cited. (cc) BY 


\section{References}

[1] Penyusun T. Adat Istiadat Daerah Nusa Tenggara Barat. Jakarta: Departemen Pendidikan dan Kebudayaan; 1977

[2] May A, Ali Y, Mahrip, Winangun, Yah, Mimbarman Duliun M. Tata Kelakukan Di Lingkungan Pergaulan Keluarga Dan Masyarakat Nusa Tenggara Barat. Jakarta: Departemen Pendidikan dan Kebudayaan; 1989

[3] Lukman L. Tata Budaya Adat Sasak Di Lombok; 2006

[4] Yasin N. Hukum Perkawinan Islam Sasak. Malang: UIN Malang Press; 2008

[5] Satrya HD, Dharma. Feudalism versus Capitalism: The changing representation of Lombok in Nadira Khalid's Novel Ketika Cinta Tak Mau Pergi. 3 L: Language, Linguistics, Literature. 2018;24(4):115-127. DOI: 10.17576/3L-2018-2404-09

[6] Kurnia MD. Warna Lokal Melayu Pada Novel Ayah Karya Andrea Hirata. Deiksis. 2015:141-163

[7] Soedjijiono. Menuju Teori Sastra Indonesia: Membangun Teori Prosa Fiksi Berbasis Novel-Novel Kearifan Lokal. Atavisme. 2009;12(1):47-63

[8] Suroso. Kepriyayian Tokoh Dalam Novel Warna Lokal Jawa Dan Sumbangannya Dalam Pengembangan Karakter Bangsa. Litera. 2011;10(2):183-191

[9] Suwignyo H. Makna Kearifan Budaya Jawa Dalam Puisi Pariksit, Telinga, Dongeng Sebelum Tidur, Dan Asmaradana. Bahasa Dan Seni. 2013;41(2):181-190

[10] Wildan. Kearifan Lokal Dalam Novel Seulusoh Karya D. Kemalawati. Bahasa Dan Seni. 2013;41(1):30-39
[11] Sesnic J. From Shadow to Presence: Representation of Ethnicity in Contemporary American Literature. New York: Rodopi; 2007

[12] Satrya HD, Dharma F, Pujiharto. Romanticism and new awareness in Indonesian literature: Lombok's representation in Novel Opto Ergo Sum. In: English Language and Literature International Conference. 2019. pp. 341-349. DOI: $10.4108 /$ eai.27-4-2019.2285292

[13] Wijaya H. Merpati Kembar Di Lombok Karya Nuriadi (Kajian Sosiologi Sastra, Budaya, Resepsi, Dan Nilai Pendidikan). Universitas Sebelas Maret; 2013

[14] Zuhairini S. Idealisme Perjuangan Perempuan Sasak Dalam Novel 'Merpati Kembar Di Lombok' Karya Nuriadi Dan Relevansinya Terhadap Pembelajaran Sastra Di SMAN 1 Kediri. Mabasindo. 2017;1(1):75-84

[15] Zuhdi MH. Praktik Merariq: Wajah Sosial Orang Sasak. Lembaga Pengkajian-Publikasi Islam \& Masyarakat (LEPPPIM) IAIN Mataram; 2012

[16] Lukman W. Eksistensi Perkawinan Masyarakat Suku Sasak Lombok (Merariq) Dalam Muara Pluralisme Hukum. IUS. 2014;II(6 Desember II):427-444

[17] Basriadi. Tinjauan Hukum Islam Terhadap Perkawinan Beda Kelas Muslim Sasak Di Lombok. Maraji: Jurnal Studi Keislaman. 2015;1(2):297-329

[18] Aminullah MN. Akulturasi Islam Dengan Tradisi Perkawinan Masyarakat Bangsawan Sasak (Studi Di Kecamatan Kopang Kabupaten Lombok Tengah). Palapa: Jurnal Studi Keislaman. 2017;5(1):109-137 
"Kidnapping the Bride"-A Traditional Sasak Wedding Seen in Sesak Cinta Di Tanah Sasak... DOI: http://dx.doi.org/10.5772/intechopen.93697

[19] Mahayanti IR. Pemberian Pisuke Dalam Perkawinan Adat Masyarakat Sasak Di Desa Dasan Tereng Kec. Narmada Kabupaten Lombok Barat. Unversitas Gadjah Mada; 2017

[20] Krulfeld R. Fatalism in Indonesia: A comparison of socio-religious types on lombok. Anthropological Quarterly. 1966;39(3):180-190

[21] Budiwanti E. Islam Sasak: Waktu Telu versus Waktu Lima. Yogyakarta: LKIS; 2000

[22] Nahdi K. In: Purnama S, editor. Nahdlatul Wathan Dan Peran Modal: Studi Etnografi Historis Modal Spiritual Dan Sosiokultural. Yogyakarta: Insyira; 2012

[23] Suhardi, Yasri H, Mursip. Upacara Daur Hidup Suku Sasak. Mataram: Pustaka Widya; 2010

[24] Hadi F. Potret Kehidupan

Masyarakat Sasak. Lombok Timur: KSU Primaguna; 2014

[25] Mansyur Z. Tradisi Maulid Nabi Dalam Masyarakat Sasak. Ulumuna IX. 2005;1:90-103

[26] Hunter CL. Local issues and changes: The post-new order situation in rural lombok. Sojourn: Journal of Social Issue in Southeast Asia. 2004;19(1):100-122

[27] Asnawi. Respons Kultural Masyarakat Sasak Terhadap Islam. Ulumuna. 2005;IX(1):1-19

[28] Syakur AA. In: Herawati, editor. Islam Dan Kebudayaan: Akulturasi Nilai-Nilai Islam Dalam Budaya Sasak. Yogyakarta: Adab Press Fak. Adab UIN Sunan Kalijaga Yogyakarta; 2006

[29] Macdougall Johan M. 2007. Criminality and the political economy of security in Lombok. Renegotiating Boundaries. Henk Schulte Nordholt, Gerry Van Klinken, 281-304. Brill
[30] Kristiansen S. Violent youth groups in Indonesia: The cases of Yogyakarta and Nusa Tenggara Barat. Sojourn: Journal of Social Issue in Southeast Asia. 2003;18(1):110-138

[31] Kingsley J. Pelopor Perdamaian Atau Perusak Perdamaian?: Pemilihan Kepala Daerah, Kepemimpinan Agama, Dan Proses Perdamaian Di Lombok. In: Susanto AF, editor. Kegalauan Identitas: Agama, Etnisitas, Dan Kewarganegaraan Pada Masa PascaOrde Baru. Jakarta: Gramedia; 2011. pp. $97-118$

[32] Mubarok MZ. NTB Bersaing. BAPEDA: Mataram; 2009

[33] Fadjri M. Mentalitas Dan Ideologi

Dalam Tradisi Historigrafi Sasak

Lombok Pada Abad XIX-XX. Universitas Gadjah Mada; 2015

[34] Turmudji A. Sesak Cinta Di Tanah Sasak. Mataram: CV Dimensi Raya; 2010

[35] Jamaludin. Sejarah Islam Lombok: Abad Ke-16 Hingga Abad Ke-20. LEPPIM IAIN Mataram: Mataram; 2019

[36] Alaini NN. Stratifikasi Sosial Masyarakat Sasak Dalam Novel Ketika Cinta Tak Mau Pergi Karya Nhadira Khalid. Kandai 11 nomor. 2015;1:110-123

[37] Faruk. Kisah Penjara Etis Dan Filosofis: Analisis Lintas Budaya Atas 'Tembok Tidak Tinggi' Karya Samad Ismail Dan 'Merek Yang Dilumpuhkan' Karya Pramodya Anantatur.

Humaniora. 2008;20(2):224-235

[38] Supriyadi. Perkembangan Teori Strukturalisme Dalam Kritik Sastra Akademik Di Universitas Gadjah Mada Dan Universitas Indonesia Sejak 1980an Sampai 2000an. In: Sudibyo, Anwar S, editors. Mencari Formula Baru Kritik Sastra Indonesia. Interlude: Yogyakarta; 2017. pp. 54-64 
[39] Faruk. Metode Penelitian Sastra, Sebuah Penjelajahan Awal; 2012

[40] Kuhn TS. The Structure of Scientific Revolutions; Peran Paradigma Dalam Revolusi Sains. 7th ed. Bandung: Remaja Rosdakarya; 2012

[41] Shri A-PH. Paradigma Profetik Islam, Epistemologi, Etos, Dan Model. Yogyakarta: Universitas Gadjah Mada Press; 2016

[42] Saukko P. Doing Research in Cultural Studies. London: SAGE Publication; 2003 



\section{Edited by Dhastagir Sultan Sheriff}

With the emerging newer trends in sexuality and clashes with the traditional practice of human sexuality, there is a need for introspection and a framework of ethical principles that may guide human sexuality and its practice. Human sexuality is more than a biological phenomenon. Open discussions about sexual identity and sexual practices will help people better understand themselves, others, and the world around them. This book gives a panoramic view of certain aspects of human sexuality in health and disease. 